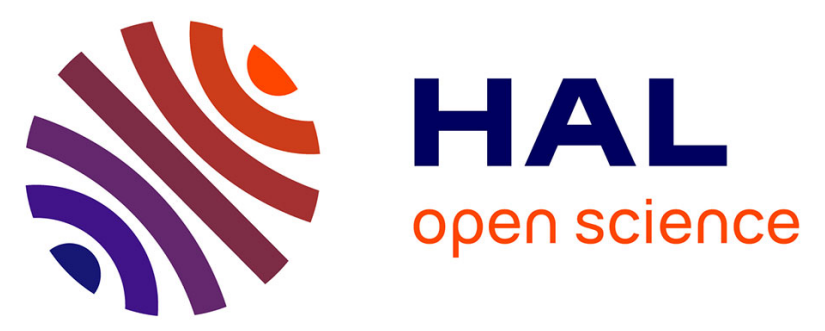

\title{
Reversing Reactivity: Stereoselective Desulfurative 1,2- trans-O-Glycosylation of Anomeric Thiosugars with Carboxylic Acids under Copper or Cobalt Catalysis
}

Nedjwa Bennai, Amélie Chabrier, Maha Fatthalla, Christine Tran, Expédite Yen-Pon, Mohamed Belkadi, Mouad Alami, Laurence Grimaud, Samir Messaoudi

\section{To cite this version:}

Nedjwa Bennai, Amélie Chabrier, Maha Fatthalla, Christine Tran, Expédite Yen-Pon, et al.. Reversing Reactivity: Stereoselective Desulfurative 1,2- trans-O-Glycosylation of Anomeric Thiosugars with Carboxylic Acids under Copper or Cobalt Catalysis. Journal of Organic Chemistry, 2020, 85 (14), pp.8893-8909. 10.1021/acs.joc.0c00766 . hal-03036397

\section{HAL Id: hal-03036397 https://hal.science/hal-03036397}

Submitted on 2 Dec 2020

HAL is a multi-disciplinary open access archive for the deposit and dissemination of scientific research documents, whether they are published or not. The documents may come from teaching and research institutions in France or abroad, or from public or private research centers.
L'archive ouverte pluridisciplinaire HAL, est destinée au dépôt et à la diffusion de documents scientifiques de niveau recherche, publiés ou non, émanant des établissements d'enseignement et de recherche français ou étrangers, des laboratoires publics ou privés. 


\title{
Reversing reactivity: stereoselective desulfurative 1,2-trans- O-glycosylation of anomeric thiosugars with carboxylic acids under copper or cobalt catalysis
}

\author{
Nedjwa Bennai, ${ }^{[a][b] \dagger}$ Amélie Chabrier, ${ }^{[a] \dagger}$ Maha I. Fatthalla,${ }^{[a][c]}$ Christine Tran, ${ }^{[a]}$ Expédite Yen-Pon, ${ }^{[a]}$ \\ Mohamed Belkadi, ${ }^{[\mathrm{b}]}$ Mouâd Alami, ${ }^{[\mathrm{a}]}$ Laurence Grimaud ${ }^{[\mathrm{d}]}$ and Samir Messaoudi ${ }^{*[\mathrm{a}]}$

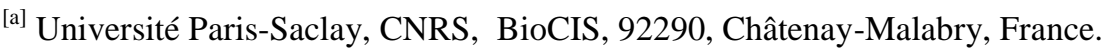 \\ ${ }^{\text {b] }}$ Université des sciences et de la technologie d'Oran-Mohamed-Boudiaf, Algeria \\ ${ }^{[c]}$ Department of Chemistry, Faculty of Science, Helwan University, 11795 Ain Helwan, Cairo, Egypt \\ ${ }^{[d]}$ Laboratoire des biomolécules (LBM), Sorbonne Université - Ecole Normale Supérieure - CNRS, 24 rue Lhomond, 75005 , \\ Paris, France. \\ $\dagger$ contributed equally.
}

KEYWORDS. thiosugars $\bullet \beta-\mathrm{O}$-glycosylation $\bullet \beta$-O-glycosyl esters $\bullet$ copper $\bullet$ cobalt $\bullet$ carbohydrates

\begin{abstract}
We have discovered a new mode of reactivity of 1-thiosugars in the presence of $\mathrm{Cu}$ (II) or Co(II) for a stereoselective $\mathrm{O}$-glycosylation reaction. The process involves the use of a catalytic amount of $\mathrm{Cu}(\mathrm{acac})_{2}$ or $\mathrm{Co}(\mathrm{acac})_{2}$ and $\mathrm{Ag}_{2} \mathrm{CO}_{3}$ as an oxidant in $\alpha, \alpha, \alpha$-trifluorotoluene (TFT). Moreover, this protocol turned out to have a broad scope, allowing to prepare a wide range of complex substituted $O$-glycoside esters in good to excellent yields with an exclusive 1,2-trans-selectivity. The late-stage modification of pharmaceuticals by this method was also demonstrated. To get a closer insight into the reaction mechanism, cyclic voltammetry (CV) was performed.
\end{abstract}

Introduction

Sugars are a very important family of biomolecules that play a pivotal role in living organisms. Their inherent structural complexity enables them to interact with myriad of biological receptors. ${ }^{1}$ To understand the role of saccharides in biological systems, chemists need to prepare well-defined chemical glycosides. $\beta$-Acyl $O$-glycosides (AGs) is one of the common motif found in natural products or bioactive compounds such as phyllanthoside, ${ }^{2}$ neurosporaxanthin, ${ }^{3}$ cephasinenoside, ${ }^{4}$ macrophylloside $D^{5}$, celecoxib acyl- $\beta$-D-glucuronide metabolite $^{6}$ (Figure 1) and the immunoadjuvant QS-21A . Numerous $^{7}$ members of the ellagitannin family also have a 1acylglycoside unit, such as sanguiin $\mathrm{H}-4$ and sanguiin $\mathrm{H}-5 .{ }^{8}$ The use of AGs as surfactants also appeared to be an interesting strategy to increase the lignocellulosic biomass value.' Recently mannosamine lipid esters ( $\mathrm{Ac}_{4} \mathrm{ManNAz}$ lipid ester, Figure 1) were used as chemical tools for metabolic cell labeling strategy. ${ }^{10}$ Moreover, $\beta$-1-acyl glucuronides are the major metabolites of most carboxylic acid containing drugs. ${ }^{11}$ Several $\beta$-1-acyl glucosides were also identified as metabolites of bile acids and important bio-markers for patients with hepatic diseases. ${ }^{12}$ More importantly, glycoacylation has been pointed out as an attractive pathway to target cancer cells exploiting the Warburg effect. ${ }^{13}$ This antitumor strategy that gained much more attention in the last decades, is based on the use of several glucose-based conjugates to more specifically deliver the attached drug to cancer cells. There have been many examples of glucose-conjugated drugs in preclinical or clinical evaluation (paclitaxel, ${ }^{14}$ adriamycin, ${ }^{15}$ DNA alkylating agent

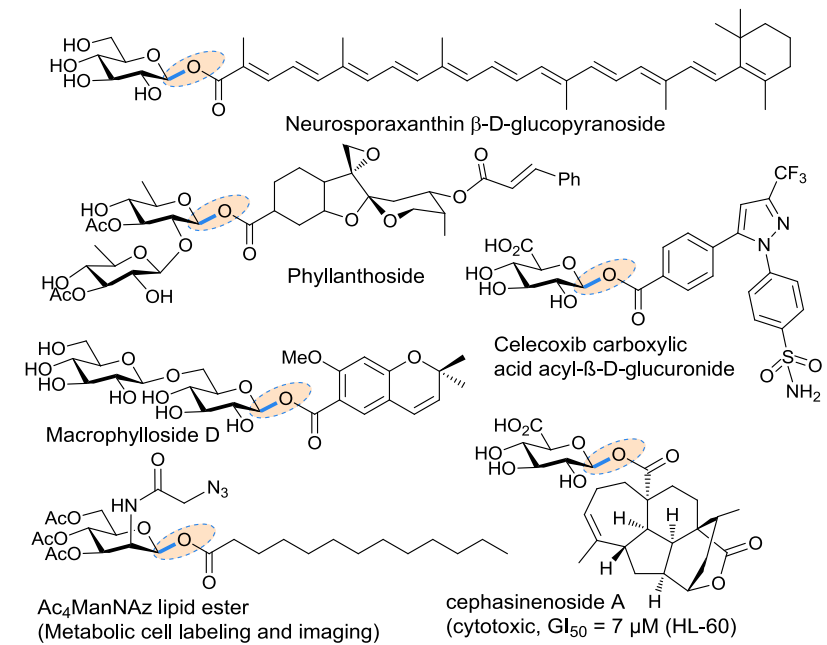

Figure 1. Examples of natural products and bioactive compounds bearing a 1-acyl sugar moiety.

including chlorambucil, ${ }^{16}$ platinum $^{17}$ and cyclophosphamide $^{18}$ ).

Despite the high added-value of these substrates, a general catalytic-stereoselective acylation method of sugars has not been yet developed. Thus, it has become critical to stereoselectively prepare $\beta$-1-acyl glycosides. In vertebrates, acyl $O$-glucuronide are synthesized by the conjugation of carboxylic acids with uridine diphosphate glucuronic acid (UDPGA) mediated by various glucuronosyl transferases (UDPGTs) (Figure 2). ${ }^{19}$ From a synthetic point-of-view, usually, these 


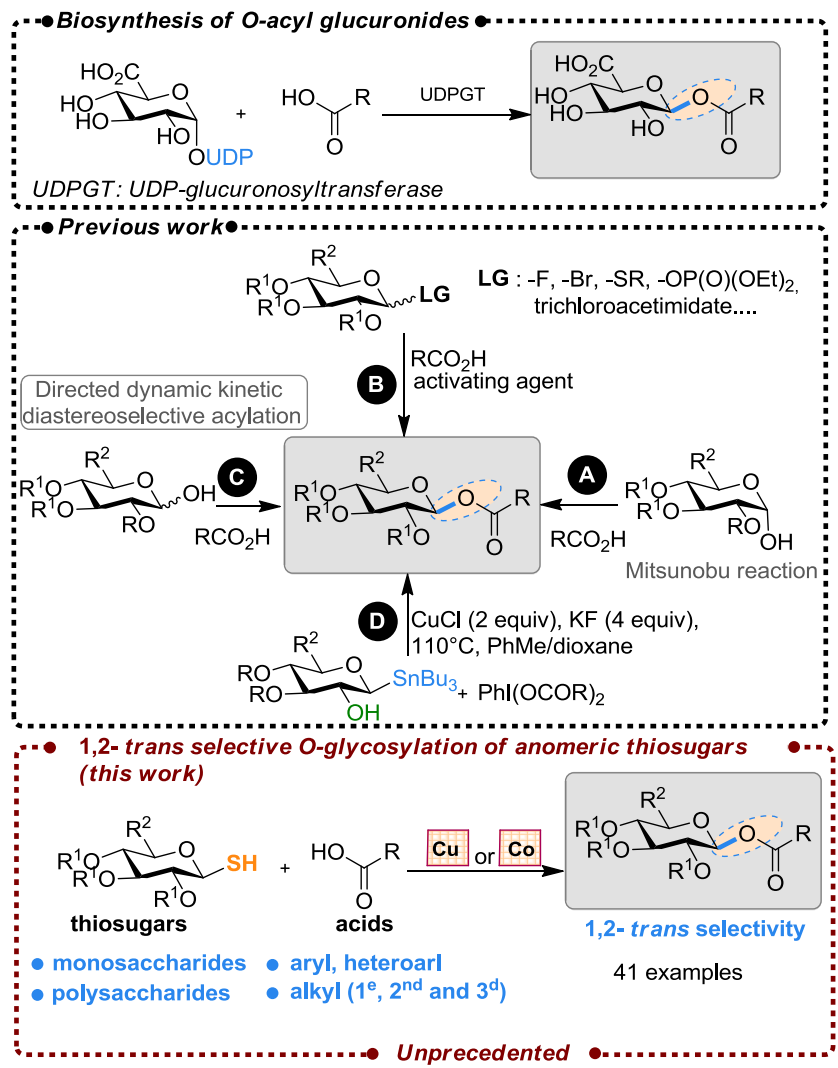

Figure 2. Strategies for $O$-glycosylation to access to acyl-Oglycosides.

derivatives were prepared by treating benzoic acid derivatives with sugar lactols through an $\mathrm{S}_{\mathrm{N}} 2$ Mitsunobu reaction ${ }^{20}$ (Figure 2, path A). However a mixture of anomers were obtained in several cases. Some specific acyl glucuronides can be prepared through $\beta$-acylation of allyl glucuronate with carboxylic acids catalyzed by $\mathrm{HATU}^{21}$ and then mild deprotection after treatment with $\mathrm{Pd}\left(\mathrm{PPh}_{3}\right)_{4}$ in the presence of morpholine. However, the use of glycosyl lactols in all these strategies remains problematic because of the difficulty to prepare well-defined $\alpha$ - or $\beta$-anomers. Another way to prepare acyl-O-glycosides is the use of glycosyl donors such as glycocosyl halides, thioglycosides, glycosyl diethyl phosphates and glycosyl trichloroacetimidates as partners in the glycosylation reaction with carboxylic acids ${ }^{22}$ (Figure 2, path B). However, these methods suffer from several drawbacks such as the limited scope to non-functionalized substrates, the need for lengthy multisteps preparation of the donor, and the low stereoselectivity of $\mathrm{C} 1$ conformation.

One different approach toward acyl-glycoside is the directed dynamic kinetic stereoselective acylation of anomeric hydroxyl groups by using a chiral catalyst ${ }^{23}$ (Figure 2, path C). Recently, Walczak group reported an elegant method to acylate glycosyl stannane donors ${ }^{24}$ (Path D). This copper-mediated $O$ glycosylation reaction involved the coupling of anomeric stannane nucleophiles with carboxylic acids resulting in exclusive anomeric selectivities. Although this method showed a broad scope, it however requires a multi-steps procedures to prepare the 1-stanylated sugar substrates. Moreover, the toxicity of the organic tin species remain always a major concern. Because of all these drawbacks, developing complementary alternatives are highly desirable for this seemingly simple transformation. In this work we showed for the first time, that thiosugars and acids can be joined together through a desulfurative 1,2-trans-glycosylation process to afford stereoselectively a variety of functionalized acyl- 1,2-transglycosides. One of the advantages of this approach in addition to the broad scope tolerance, is the availability of thiosugars: these substrates are commercially available or may be synthesized easily from the peracetylated precurssors in three-steps.

During the course of investigating the reactivity of various thiosugars toward $\mathrm{C}-\mathrm{H}$ activation processes ${ }^{26}$ we subjected tetra-O-acetylated-1-thio- $\beta$-D-glucopyranose 1a and aliphatic amide $\mathbf{4 a}$ to the $\mathrm{Cu}$-catalyzed functionalization of unactivated $\mathrm{Csp}^{3}-\mathrm{H}$ bonds under previously reported conditions. ${ }^{27}$ Rather than obtaining the expected $\mathrm{Csp}^{3}-\mathrm{S}$ bond formation (compound $\mathbf{6 a}$ ), an $O$-glycosylation process was serendipitously discovered leading to compound $\mathbf{7 a}$ in $10 \%$ yield as a pure $\beta$ isomer (Scheme 1).

Scheme 1: Unexpected $O$-glycosylation observed when using $\beta$-thioglucose 1a with benzoic acid $\mathbf{5 a}$.

Early observation: Unexpected O-glycosylation observed when using thiosugar in $\mathrm{C}\left(\mathrm{sp}^{3}\right)-\mathrm{H}$ bond activation in the presence of $\mathrm{PhCO}_{2} \mathrm{Na}$

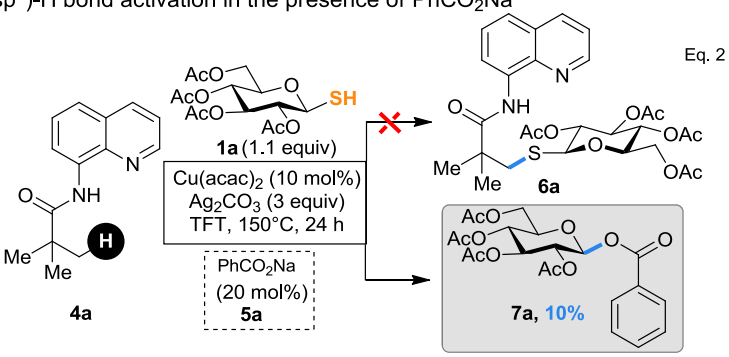

We hypothesized that formation of $7 \mathbf{a}$ resulted from reaction of sodium benzoate, which was used in a catalytic amount (20 mol \%), with the $\beta$-thioglucose 1a. We therefore decided to explore the viability of this unusual $O$-glycosylation of thiosugars. Herein, we report the optimization of this reaction which appears as a new method for the 1,2-transstereoselective $O$-glycosylation of thiosugars.

Results and Discussion

When conducting the reaction of 1a with 1 equivalent of benzoic acid $\mathbf{5 a}$ in the absence of the amide $4 \mathbf{a}, O$-glycosylation was still observed and the $\beta-O$-acyl glucoside 7a was isolated in $29 \%$ yield (Table 1, entry 2). The exact structure of $\mathbf{7 a}$, including its 1,2-diaxial geometry, was determined by $1 \mathrm{D}$ and 2D NMR spectroscopy and unambiguously confirmed by crystal structure analysis (Figure S10 in SI). Then, an optimization study was carried out using $\beta$-thioglucose 1a and benzoic acid 5a as substrates (details can be found in Table S1 in the Supporting Information). To understand the observed $O$ glycosylation reaction and to verify that the outcome was derived from a catalytic process, several control studies were performed (Table 1). These studies led us to identify the best reaction conditions as the use of $5 \mathrm{~mol} \% \mathrm{Cu}(\mathrm{acac})_{2}$ and 3 equivalents of $\mathrm{Ag}_{2} \mathrm{CO}_{3}$ in trifluorotoluene (TFT, $0.1 \mathrm{M}$ ) at 130 ${ }^{\circ} \mathrm{C}$ under microwave irradiation for 15 minutes. Under these conditions, the desired phenyl acylglycoside $\mathbf{7 a}$, was isolated in $79 \%$ yield (Table 1, entry 1). Running the reaction in oil bath at $130{ }^{\circ} \mathrm{C}$ for $1 \mathrm{~h}$ instead of microwave irradiation led to the desired product in only $45 \%$ yield (entry 3 ). Moreover, performing the reaction in oil bath at $150^{\circ} \mathrm{C}$ for $1 \mathrm{~h}$ furnished 7a in $74 \%$ yield (entry 4). Interestingly, we observed during the optimization that the copper catalyst $\mathrm{Cu}(\mathrm{acac})_{2}$ can be replaced by $\mathrm{Co}(\mathrm{acac})_{2}$ without affecting the yield (77\% yield, entry 8). We also confirmed that $\mathrm{Ag}_{2} \mathrm{CO}_{3}$ is required to accomplish $O$-glycosylation; without $\mathrm{Ag}_{2} \mathrm{CO}_{3}$ (entry 5,6) or 
performing the reaction with other bases $\left(\mathrm{K}_{2} \mathrm{CO}_{3}\right.$ or $\left.\mathrm{Cs}_{2} \mathrm{CO}_{3}\right)$ instead of silver carbonate, no reaction occurred (entries 6 and 11). Interestingly, control experiment showed also that this reaction could be performed without catalyst, but 7a was isolated in a lower yield (52\%, entry 7$)$. In addition, reaction with $\mathrm{Cu}(\mathrm{I})$ was less efficient and gave product $\mathbf{7 a}$ in a yield similar to the reaction without catalyst (46\%, entry 9). We also checked whether the copper salt could act as a Lewis acid by performing the reaction in the presence of a catalytic amount of the Lewis acid $\mathrm{AlCl}_{3}(5 \mathrm{~mol} \%)$ instead of the copper catalyst $\mathrm{Cu}(\mathrm{acac})_{2}$ (entry 10). However under these conditions, only $59 \%$ yield of the desired product were obtained, suggesting that this pathway plays only a minor role. Finally, when the reaction was performed in the poorly absorbing toluene solvant, compound 7a was obtained in a lower yield $(67 \%$ instead $79 \%$ with TFT, entry 12 vs entry 1 ). This difference maybe is due to the particular properties of trifluorotoluene (dielectric constants and dipole moments) which are more adaptable to the microwave activation mode.

Table 1 Reaction conditions optimization for the $O$-glycosylation of tetra- $O$-acetylated-1-thio- $\beta$-D-glucopyranose $1 \mathrm{a}$ with phenylbenzoïc acid 5a. ${ }^{a}$

\begin{tabular}{|c|c|c|}
\hline & 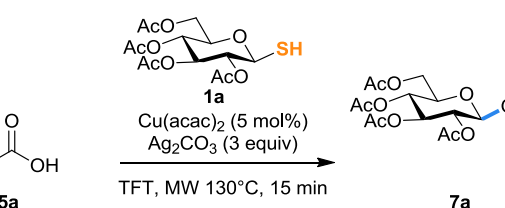 & \\
\hline Entry & Deviation from the standard conditions & $\begin{array}{l}\text { Yield } \\
(\%)^{b}\end{array}$ \\
\hline 1 & none & 79 \\
\hline 2 & $12 \mathrm{~h}$ at $150{ }^{\circ} \mathrm{C}$ (oil bath) & 29 \\
\hline 3 & $1 \mathrm{~h}$ at $130^{\circ} \mathrm{C}$ (oil bath) & 45 \\
\hline 4 & $1 \mathrm{~h}$ at $150{ }^{\circ} \mathrm{C}$ (oil bath) & 74 \\
\hline 5 & without $\mathrm{Cu}$-catalyst and $\mathrm{Ag}_{2} \mathrm{CO}_{3}$ & 0 \\
\hline 6 & without $\mathrm{Ag}_{2} \mathrm{CO}_{3}$ & 0 \\
\hline 7 & without $\mathrm{Cu}$-catalyst & 52 \\
\hline 8 & $\mathrm{Co}(\mathrm{acac})_{2}$ instead of $\mathrm{Cu}(\mathrm{acac})_{2}$ & $77^{c}$ \\
\hline 9 & $\mathrm{CuI}$ instead of $\mathrm{Cu}(\mathrm{acac})_{2}$ & 46 \\
\hline 10 & $\begin{array}{c}\mathrm{AlCl}_{3}(5 \mathrm{~mol} \%) \text { Lewis acid instead } \\
\text { of } \mathrm{Cu}(\mathrm{acac})_{2}\end{array}$ & 59 \\
\hline 11 & $\mathrm{~K}_{2} \mathrm{CO}_{3}$ or $\mathrm{Cs}_{2} \mathrm{CO}_{3}$ instead of $\mathrm{Ag}_{2} \mathrm{CO}_{3}$ & 0 \\
\hline 12 & Toluene instead of TFT & 67 \\
\hline
\end{tabular}

TFT $=$ trifluorotoluene ${ }^{a}$ Conditions: 1a (1.5 equiv), 5a ( 1 equiv), metal source ( $5 \mathrm{~mol} \%)$, base ( 3 equiv), TFT $[0.1 \mathrm{M}]$ were heated in a sealed tube under argon atmosphere. ${ }^{b}$ Yield of isolated $7 \mathbf{a} .{ }^{c} 1 \mathrm{~h}$ reaction time under thermal heating (at $150^{\circ} \mathrm{C}$ ).

Prompted by these exciting results, we subsequently investigated the substrate scope for the catalytic $O$-glycosylation of tetra- $O$-acetylated-1-thio- $\beta$-D-glucopyranose 1 a with various commercial carboxylic acids 5a-z, 5aa-ac using either $\mathrm{Cu}(\mathrm{acac})_{2}$ or $\mathrm{Co}(\mathrm{acac})_{2}$. In most examples, yields arising from $\mathrm{Cu}$ - and Co-catalysis are provided, otherwise, in other cases, only one catalyst was used. Overall, the method works well and tolerates a large variety of acid partners (Table 2), and comparable yields were obtained with both copper and cobalt catalysts (Table 2). Benzoïc acids having electron-donating groups (5b-h) led to the formation of corresponding glycosyl esters $\mathbf{7 b}$-h in good yields, up to $85 \%$. Delightfully, this protocol was efficient starting from benzoïc acids bearing free hydroxyl and amino groups on the aromatic ring (compounds
Table 2. Scope of commercial carboxylic acids $\mathbf{5 a - z}$ in the $O$ glycosylation of $\beta$-thioglucose 1a

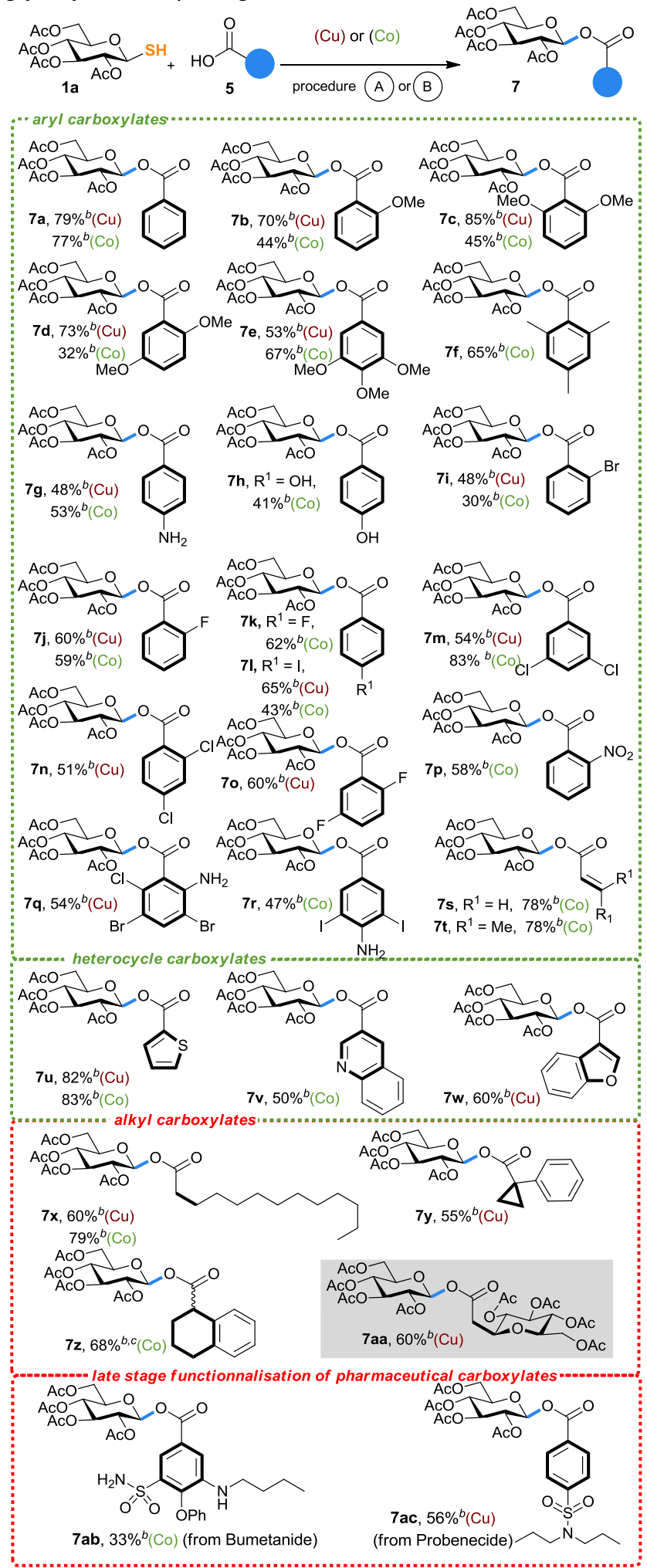

[a] Reactions conditions: procedure (A) with $\mathrm{Cu}$ : carboxylic acids 5 (1 equiv), $O$-acetylated 1 -thio- $\beta$-D-glucose $1 \mathrm{a}$ (1.5 equiv), $\mathrm{Cu}(\mathrm{acac})_{2}(5 \mathrm{~mol}$ $\%), \mathrm{Ag}_{2} \mathrm{CO}_{3}$ (3 equiv), TFT $[0.1 \mathrm{M}]$ in a sealed tube under argon at $130^{\circ} \mathrm{C}$ under microwave irradiation for 15 min. Procedure (B) with Co: carboxylic acids 5 (1 equiv), $O$-acetylated 1-thio- $\beta$-D-glucose 1a (1.5 equiv), $\mathrm{Co}(\mathrm{acac})_{2}$ (5 or $\left.10 \mathrm{~mol} \%\right), \mathrm{Ag}_{2} \mathrm{CO}_{3}$ (3 equiv), TFT $[0.1 \mathrm{M}]$ in a sealed tube under argon at $150{ }^{\circ} \mathrm{C}$ for 1 hour under thermal conditions. ${ }^{b}$ yield for the isolated product. ${ }^{c}$ diastereomeric mixture $1: 1$ calculated by ${ }^{1} \mathrm{H}$ NMR. 
$\mathbf{7 g}, \mathbf{7 h}, \mathbf{7 q}$ and $\mathbf{7 r}$ ). Likewise, 1-thio- $\beta$-D-glucose 1a was readily coupled with carboxylic acids derivatives bearing electronwithdrawing group (e.g., $\left.-\mathrm{NO}_{2},-\mathrm{F},-\mathrm{Cl},-\mathrm{Br},-\mathrm{I}\right)$ to give the $O$-glycosylated products 7i-r, which may be useful for further regioselective cross-coupling reaction concerning the halogenated substrates (Table 2). Vinyl carboxylic acids $\mathbf{5 s , t}$ are also well tolerated in this coupling leading to the acrylic $O$-acyl glycosides $\mathbf{7 s , t}$ in $78 \%$ yield. Importantly, heterocyclic acids such as thiophene, quinoleine and benzofuran reacted well with 1a, leading to products $\mathbf{7 u - w}$ in $83 \%, 50 \%$ and $60 \%$ yields, respectively. Finally, the synthetic potential of this protocol was well illustrated by its application on substrates containing alkyl groups. Thus, the tridecanoic acid $\mathbf{5 x}$, cyclopropyl carboxylic acid $\mathbf{5 y}$ as well as tetrahydronaphthalene-1- carboxylic $\mathbf{5 z}$ were good partners leading to glycosyl esters $\mathbf{7} \mathbf{x}-\mathbf{z}$ in good to excellent yields (55$79 \%)$. Interestingly, the disaccharide ester $7 \mathbf{a a}$ was obtained through this approach in $60 \%$ yield when the sugar carboxylic acid $\mathbf{5 a a}$ was used.

After having demonstrated an excellent reactivity with simple substrates, we then examined if the $O$-glycosylation reaction could be extended to drug-like small molecules through a late stage modification process. In this context, Bumetanide $\left(\right.$ Bumex $\left.^{\circledR}\right)$, a marked-loop diuretic used to treat heart failure, and probenecide (benemide ${ }^{\circledR}$ ), an uricosuric agent that inhibits the renal excretion, were successfully converted in one step into the corresponding $\beta$-acylglucosyl analogues 7ab and 7ac in synthetically useful yields (33\% and $56 \%$ yields, respectively). These new acyl glycosides may be considered as useful tool metabolites for studying their potential role in drug toxicity. They can be also deacetylated by using literature methods. ${ }^{28}$

To further demonstrate the generality of this reaction, we applied the optimized conditions to carboxylic acid, $\mathbf{5 c}$ and $\mathbf{5 m}$ and structurally diverse mono-thiosugars $\mathbf{1 b}-\mathbf{g}$ (Table 3 ). This $O$-glycosylation appeared to be not limited to 1 -thio- $\beta$ - $\mathrm{D}$ glucose: thiosugars derived from $\beta$-D-galactopyranose (1b), and $\beta$-D-fucose (1d) were also coupled with $\mathbf{5 c}$ or $\mathbf{5 m}$ to provide the corresponding $\beta$ - $O$-glycosylated esters $\mathbf{8 a}, \mathbf{b}, \mathbf{8 d}$ in good yields from either $\mathrm{Cu}$ - or Co-catalysts. Of note, the coupling of $\beta$-D-mannopyranose (1c) led as expected, to the $\alpha-O$ glycosylated ester $\mathbf{3 c}$ in which we observed the inversion of the anomeric configuration. In addition, this methodology tolerates other protecting groups such $\mathrm{OBz}$ in the disarmed donor (1e) and OMe ether (1f). Importantly, in the case of thioGlc(OMe $)_{4}(\mathbf{1 f})$, a $1: 1$ mixture of both $\alpha / \beta$ anomers was obtained indicating that the anomeric stereochemistry is controlled by the neighboring participant group at the $\mathrm{C} 2$ position. Glycosyl donors protected with tert-butyldimethylsilyl (TBS) group were well known to have superior reactivity and selectivity than other sugar donors. ${ }^{29}$ This enhanced reactivity/selectivity was correlated to the stereoelectronic effects associated with the conformation change induced by the silyl groups. Inspired by this above literature, we were wondering whether the selectivity in our glycosylation reaction will be impacted by this conformation change. To this end, we prepared the conformationally super armed OTBS-protected thiosugar (19) and we carried out the $\mathrm{Cu}$-catalyzed glycosylation reaction in the presence of phenylbenzoic acid. Interestingly, under our standard conditions the desired ${ }^{1} C_{4}$ glycoside $\mathbf{8 g}$ was obtained in good 70\% yield and 2:1 $\beta$-anomeric selectivity although no participating group was installed at $\mathrm{C}-2$ position. This result evidenced the effect of conformation change on the glycosylation selectivity. One can note that unprotected thiosugars are not tolerated under our reaction conditions.

Table 3. Scope of 1 thio- $\beta$-glycosyls $\mathbf{1 b - g}{ }^{a}$

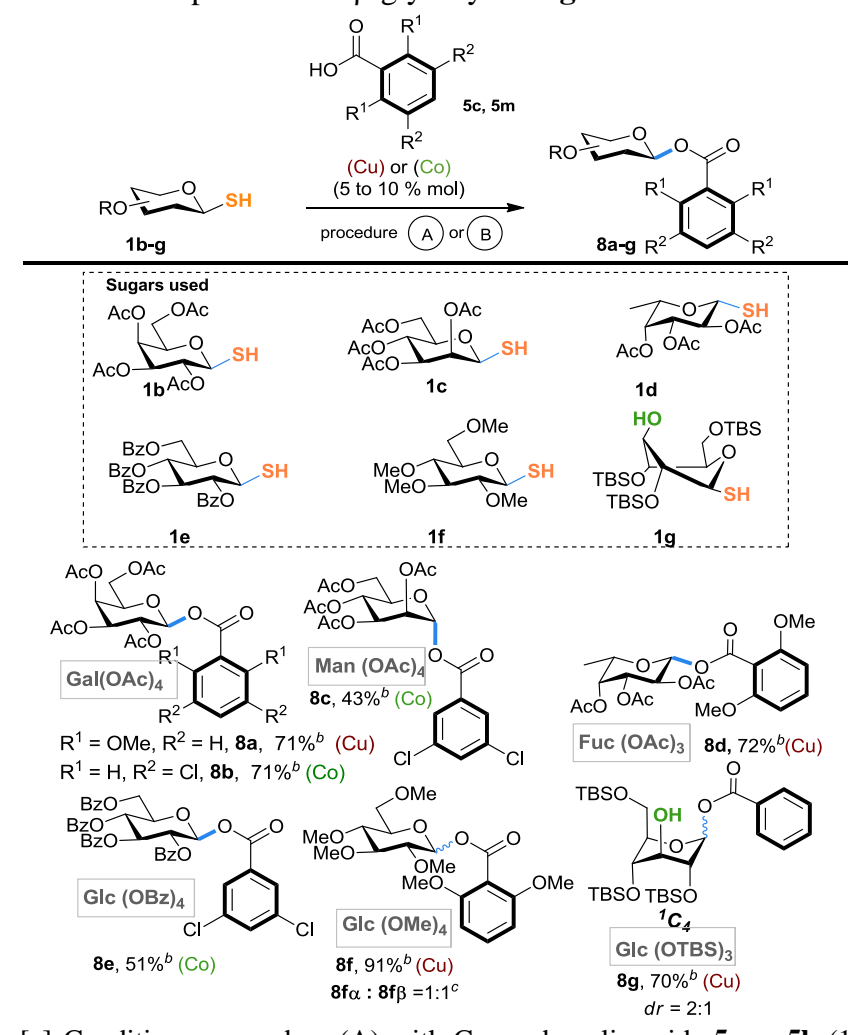

[a] Conditions: procedure (A) with $\mathrm{Cu}$ : carboxylic acids $\mathbf{5 c}$ or $\mathbf{5 k}(1$ equiv), thiosugars $\mathbf{1 b}-\mathbf{g}$ (1.5 equiv), $\mathrm{Cu}(\mathrm{acac})_{2}(5 \mathrm{~mol} \%), \mathrm{Ag}_{2} \mathrm{CO}_{3}(3$ equiv), TFT $[0.1 \mathrm{M}]$ in a sealed tube under argon at $130{ }^{\circ} \mathrm{C}$ under microwave irradiation for $15 \mathrm{~min}$. Procedure (B) with Co: carboxylic acids 5 ( 1 equiv), $O$-acetylated 1 -thio- $\beta$-D-glucose 1a (1.5 equiv), $\mathrm{Co}(\mathrm{acac})_{2}(10 \mathrm{~mol} \%), \mathrm{Ag}_{2} \mathrm{CO}_{3}$ (3 equiv), TFT [0.1 M] in a sealed tube under argon at $150{ }^{\circ} \mathrm{C}$ for 1 hour. ${ }^{b}$ yields of isolated product. ${ }^{c}$ diastereomeric mixture 1:1 calculated by ${ }^{1} \mathrm{H}$ NMR.

Having demonstrated the efficacy of our method with various thiosugars, we next turned our attention to the validation of the method with respect to more complex and biologically relevant saccharides (Scheme 2). We were pleased to find that diand trisaccharide derivatives cellobiose $\mathbf{1 h}$ and maltotriose $\mathbf{1 i}$, readily undergo $O$-glycosylation in this transformation. The desired $O$-acyl glycosides $\mathbf{8 h}$,i were isolated in $58 \%$ and $45 \%$ yields, respectively. Moreover, the $O$-acyl trisaccharide $\mathbf{8 j}, \mathbf{k}$ which are commonly used in bacterial imaging ${ }^{30}$ were isolated in synthetically useful yields.

To broaden the synthetic applications of this method, we carried out studies on whether the iodinated substrate 71 could be used as a suitable platform for introducing molecular diversity via cross coupling reactions. Thus, the glycosyl ester 9a bearing both $\mathrm{C}-\mathrm{O}$ and $\mathrm{C}-\mathrm{S} \beta$-glycosidic bonds was easily prepared through this $O$-glycosylation approach (Scheme $3 \mathrm{~A}$ ) followed by the functionalization of the $\mathrm{C}-\mathrm{I}$ bond by the coupling with the thioGluNAc $\mathbf{1 j}$ under our previously reported Pd-catalyzed methodology. ${ }^{31}$ We have also shown that this process was effective replacing thiosugar by a commercial NHAc-Cys, delivering the glycoamino acid $9 \mathrm{~b}$ in $68 \%$ yield (Scheme $3 \mathrm{~B}$ ).

With these encouraging results in hand, we next turned our attention to examine the reactivity of other thiols such as alkylthiols instead of thiosugars. Pleasantly, reaction of the commercially available $N$-acetylcysteamine with benzoic acid 
Scheme 2. Scope of complex 1 thio- $\beta$-glycosyls $\mathbf{1 h}, \mathbf{i}^{a}$

$=\mathrm{Glc}$
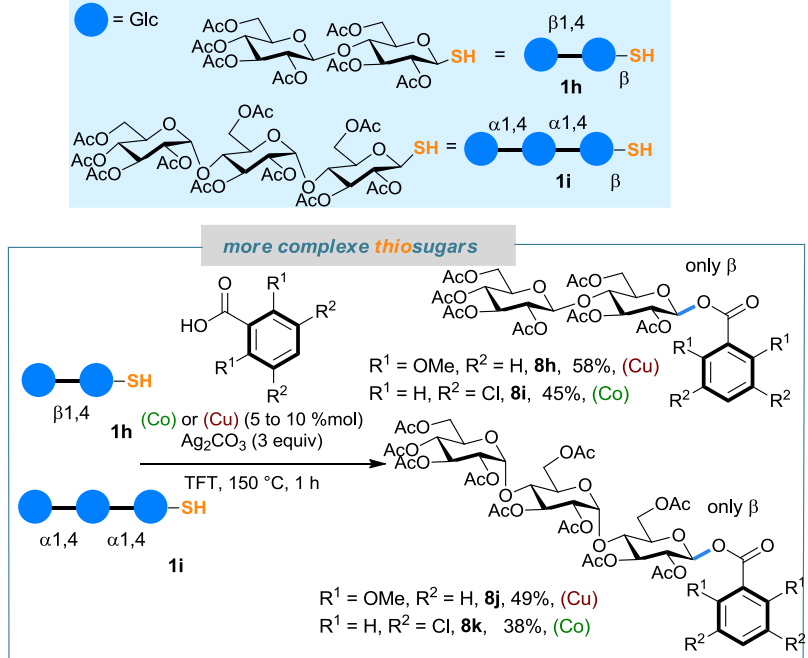

[a] Conditions: procedure (A) with $\mathrm{Cu}$ : carboxylic acids $\mathbf{5 c}$ or $\mathbf{5 m}(1$ equiv), thiosugars $\mathbf{1 h}, \mathbf{i}$ (1.5 equiv), $\mathrm{Cu}(\mathrm{acac})_{2}$ (5 mol \%), $\mathrm{Ag}_{2} \mathrm{CO}_{3}$ (3 equiv), TFT $[0.1 \mathrm{M}]$ in a sealed tube under argon at $130{ }^{\circ} \mathrm{C}$ under microwave irradiation for 15 min. Procedure (B) with Co: carboxylic acids 5 ( 1 equiv), $O$-acetylated 1 -thio- $\beta$-D-glucose $1 \mathrm{a}$ (1.5 equiv), $\mathrm{Co}(\mathrm{acac})_{2}(10 \mathrm{~mol} \%), \mathrm{Ag}_{2} \mathrm{CO}_{3}$ (3 equiv), TFT $[0.1 \mathrm{M}]$ in a sealed tube under argon at $150{ }^{\circ} \mathrm{C}$ for 1 hour. ${ }^{b}$ yields of isolated product.

$\mathbf{5 m}$ provided the ester product $\mathbf{1 0 a}$ in a $68 \%$ yield (Scheme $3 C$ ). The study of reactivity of a large variety of alkylthiols in this reaction is currently under progress in our group.

Scheme 3. Application of the methodology to molecular diversity

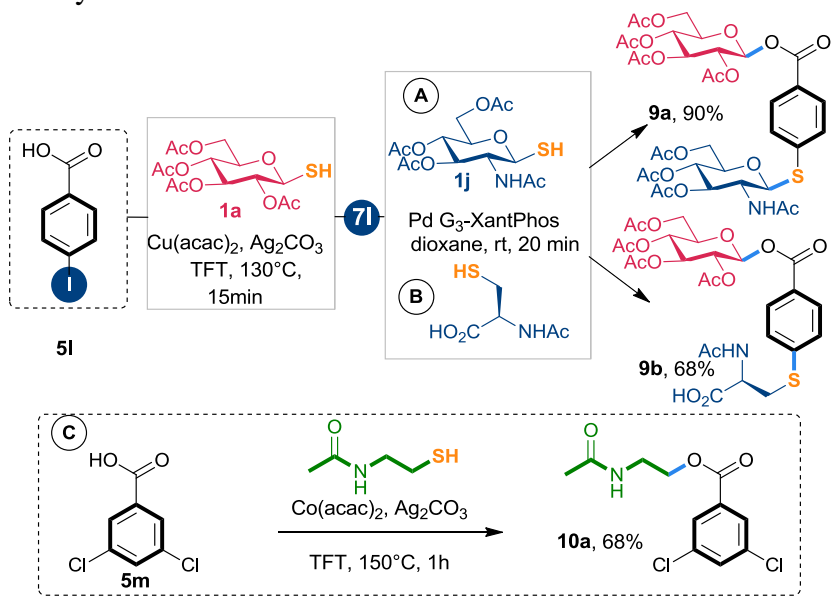

[a] Conditions: path A: $O$-acetylated 1-thio- $\beta$-D-glucose 1a $(1.5$ equiv), iodophenylbenzoic acid $\mathbf{5 l}$ (1 equiv), $\mathrm{Cu}(\mathrm{acac})_{2}(10 \mathrm{~mol} \%)$, $\mathrm{Ag}_{2} \mathrm{CO}_{3}$ (3 equiv), TFT $[0.1 \mathrm{M}]$ in a sealed tube under argon at 150 ${ }^{\circ} \mathrm{C}$ for $15 \mathrm{~min}$ under MW irradiation. path $\mathrm{B}: \mathbf{7 j}$ (1 equiv), $\mathbf{1 j}$ or NHAcCys (1.2 equiv), PdG3 XantPhos ( 5 mol \%), $\mathrm{Et}_{3} \mathrm{~N}$ ( 3 equiv), Dioxane $[0.1 \mathrm{M}]$ at $\mathrm{rt}$ for $20 \mathrm{~min}$. path C: a commercially available $N$ acetylcysteamine (1.5 equiv), benzoic acid $5 \mathrm{~m}$ (1 equiv), $\mathrm{Co}(\mathrm{acac})_{2}$ (10 mol \%), $\mathrm{Ag}_{2} \mathrm{CO}_{3}$ (3 equiv), TFT $[0.1 \mathrm{M}]$ in a sealed tube under argon at $150{ }^{\circ} \mathrm{C}$ for 1 hour.

To gain further insight into the origin of this unconventional thiol- to-acid two nucleophiles coupling, we performed the $\mathrm{C}(13)$ isotope incorporation experiment conditions by using a ${ }^{13} \mathrm{C}-\alpha$ labeled benzoic acid (99 atom $\%{ }^{13} \mathrm{C}$ ) (Scheme 4 , eq 1 ). The incorporation of ${ }^{13} \mathrm{C}$ in the phenylglycosyl ester 7a proves unambiguously that the ester-group comes from the acid function and not from a decarboxylative/carboxylation sequence with $\mathrm{CO}_{2}$ arising from the thermal decomposition of silver carbonate. $^{32}$

Scheme 4. Mechanistic investigations.

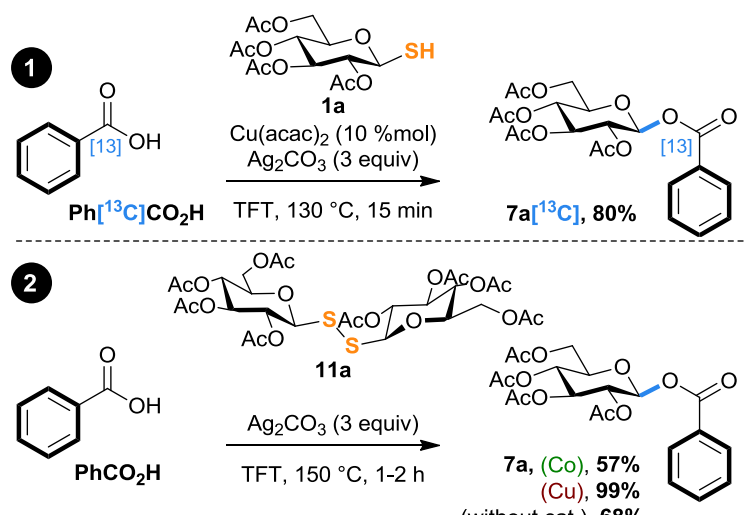

(without cat.), 68\%

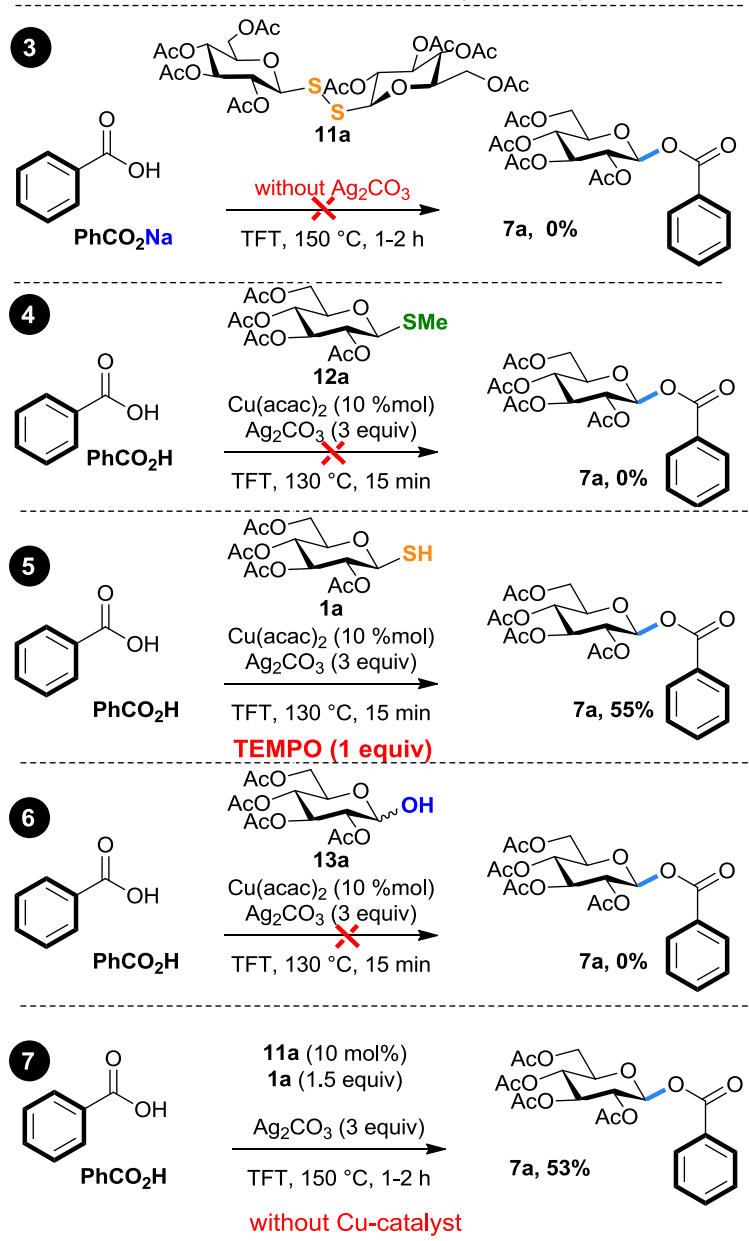

[a] Conditions: $O$-acetylated 1-thio- $\beta$-D-glucose 1a or its dimer 11a or S-methylated 12a or 13a (1.5 equiv), benzoic acid $\mathbf{2 a}$ or sodium benzoate (1 equiv), $\mathrm{Cu}(\mathrm{acac})_{2}(5 \mathrm{~mol} \%)$ or $\mathrm{Co}(\mathrm{acac})_{2}(10 \mathrm{~mol} \%)$, $\mathrm{Ag}_{2} \mathrm{CO}_{3}$ (3 equiv), solvent $[0.1 \mathrm{M}]$ in a sealed tube under argon.

Because the formation of the disulfide 11a (Scheme 4, eq.2) was observed during all the reactions (isolated and characterized), we were wondering if $\mathbf{1 1 a}$ must be regarded as a byproduct which may hamper the glycosylation process, or in contrary may be involved in the reaction pathway. In this context, we performed a series of control experiments with the disulfide 11a (Scheme 4, eq.2). Thus, heating 11a with benzo- 
ic acid $2 \mathbf{a}$ at $150{ }^{\circ} \mathrm{C}$ for $1 \mathrm{~h}$ in the presence of silver carbonate furnished the desired $\beta-O$-glycoside $7 \mathbf{a}$ in a $57 \%$ yield when the Co-catalyst was used, and $99 \%$ yield with $\mathrm{Cu}(\mathrm{acac})_{2}$ as a catalyst (Scheme 4, eq. 2). Interestingly, omitting to add the catalyst led also to the same product 7a in $68 \%$ yield. This result clearly indicates that disulfide 11a may be involved in the catalytic cycle. In addition, performing the reaction of $\mathbf{1 1 a}$ with sodium benzoate in TFT at $150{ }^{\circ} \mathrm{C}$ did not furnish $7 \mathbf{a}$ and only the starting material was recovered unchanged (Scheme 4, eq.3). This result indicates that a pathway including a simple nucleophilic substitution is discarded. In another way, the glycosylation reaction was completely inhibited when the thiol function of the sugar was protected (S-methyl glucose 12a) (Scheme 4, eq. 4). It was also found that addition of the radical inhibitor TEMPO, had no significant effect on the reaction, suggesting that a radical species may not be involved in the catalytic cycle (Scheme 4, eq. 5). Finally, the importance of the sulfhydryl $(-\mathrm{SH})$ group was further demonstrated by the Figure 3. Cyclic voltammetry $(\mathrm{CV})$ experiments

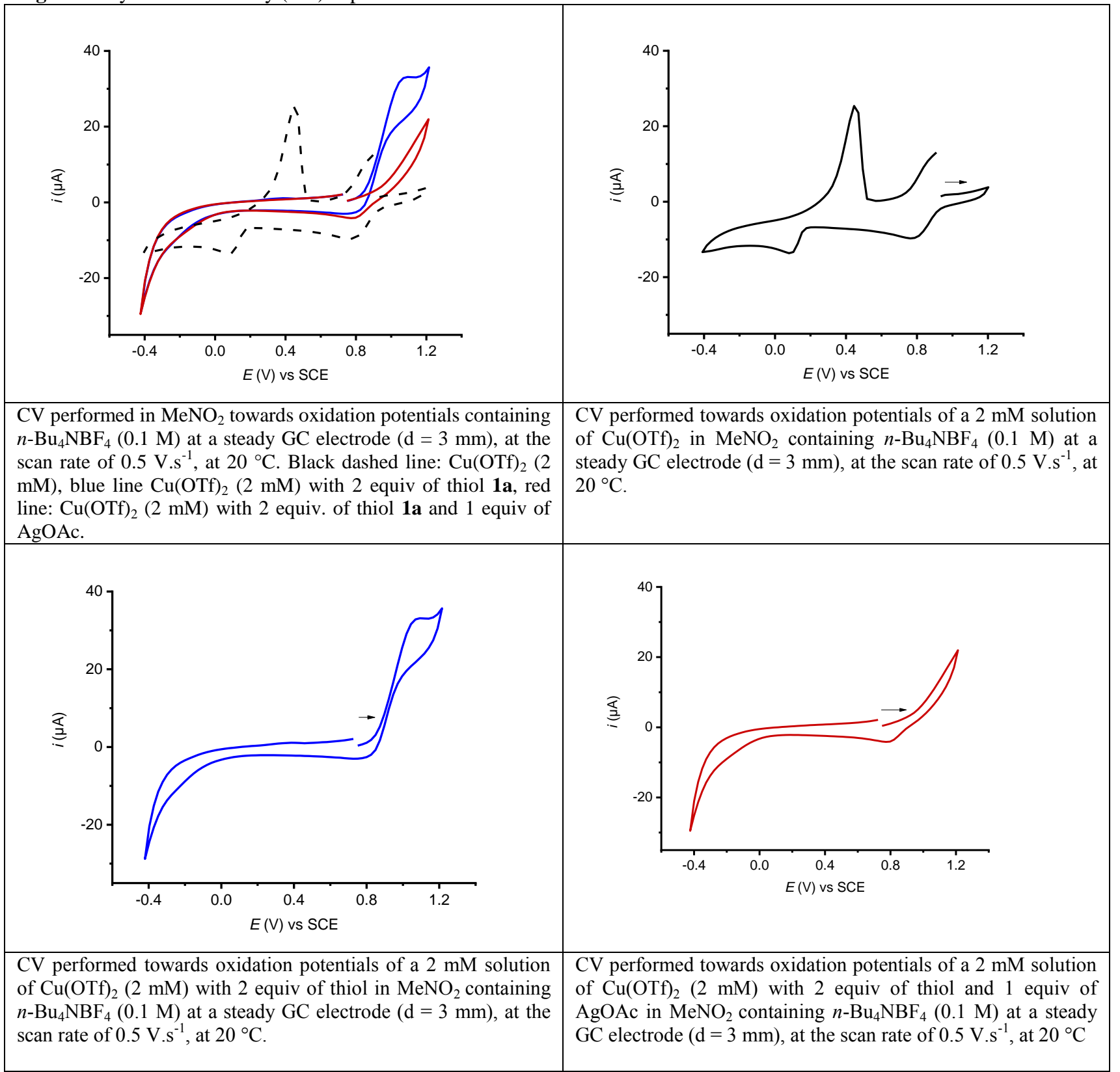

result with glycosyl lactol 13a as a sugar donor (Scheme 4, eq. $6)$. Under the standard conditions, no desired product was observed by using 13a.

The lack of any precedent for oxidative addition of thiols to $\mathrm{Cu}$-catalyst makes a traditional oxidative addition-reductive elimination pathway for this new reaction unlikely. To get a closer insight into the reaction mechanism, cyclic voltammetry $(\mathrm{CV})$ was performed (see $\mathrm{SI}) . \mathrm{Cu}(\mathrm{OTf})_{2}$ was selected as $\mathrm{Cu}(\mathrm{II})$ sources and as expected no characteristic oxidation peak could be detected towards oxidation potentials as shown with the dashed line CV (Figure 3). Just after addition of 2 equiv. of thiosugar 1a, the zero current potential of the solution was shifted by $180 \mathrm{mV}$ towards negative potentials and an oxidation peak at $+1 \mathrm{~V} v s$ SCE clearly evidenced the formation of $\mathrm{Cu}(\mathrm{I})$ as thiol is not electroactive in this range of potentials (see SI, Figure S1). A similar behavior was observed starting with $\mathrm{Cu}(\mathrm{acac})_{2}$ but the $\mathrm{CV}$ of the metal salt alone is less informative (see SI, Figure S1). The formation of $\mathrm{Cu}(\mathrm{I})$ under 
these conditions is in agreement with previous reports in the literature. ${ }^{33}$ After addition of 1 equiv of AgOAc, this oxidation peak completely disappears and a black solid precipitated, which was identified to be $\mathrm{Ag}_{2} \mathrm{~S} .{ }^{34}$ No further changes could be detected in the presence of a larger excess of $\mathbf{1 a}$ and AgOAc even in the presence of sodium benzoate (see SI, Figure S2). One can be noted that the disulfide is not electroactive in the range of potentials scanned for this study nor in the presence of a stoichiometric amount of $\mathrm{Cu}$ (II) (see SI, Figure S3).

Based on all these experiments, we propose the following mechanistic path (Figure 4). The first step involves the oxidation of thiosugar 1a by the $\mathrm{Cu}$ (II) catalyst to give the corresponding disulfide 11a (Path.1) which has a great affinity for silver. We propose that the strong complexation with silver in complex (II) weakened the carbon-sulfur anomeric bond and generated a good leaving group allowing the formation of the acetoxonium ion. The latter is trapped by the carboxylate to give the desired product 7 . Along this process, we propose that a second silver cation was involved to form the sulfonium (IV) which can be reduced by $\mathrm{Cu}$ (I) to regenerate $\mathrm{Cu}$ (II) and one thiol with formation of silver sulfide $\left(\mathrm{Ag}_{2} \mathrm{~S}\right)$ which precipitated out from the solution (see supporting information). The intermediate (IV) might regenerate 11 a by reaction with thiosugar 1a without the need of $\mathrm{Cu}$-catalyst. This step led also to the formation of the silver sulfide $\left(\mathrm{Ag}_{2} \mathrm{~S}\right)$.

Regarding the result obtained in the absence of the catalyst (Table 1, entry 7), we proposed a concomitant pathway (Path.2, Figure 3) mediated by $\mathrm{Ag}_{2} \mathrm{CO}_{3}$. The disulfide 11a might be formed in the presence of silver carbonate as an oxidant. Following a strong coordination of a second silver cation $\left(\mathrm{Ag}^{+}\right)$, the complex (II) led to the formation of an acetoxonium ion intermediate (if having a neighboring participating group at position 2). In the presence of carboxylate nucleophiles, the 1,2-trans-glycoside ester (7) could be formed producing the complex (III). This later evolves to (IV) through a second coordination with silver ion, and finally might react with 1a to regenerate the disulfide key intermediate 11a and the formation of the $\operatorname{Ag}_{2} \mathrm{~S}$. This hypothesis is in agreement with the result highlighted in Scheme 4, Eq.3; when

Figure 4. Proposed mechanism

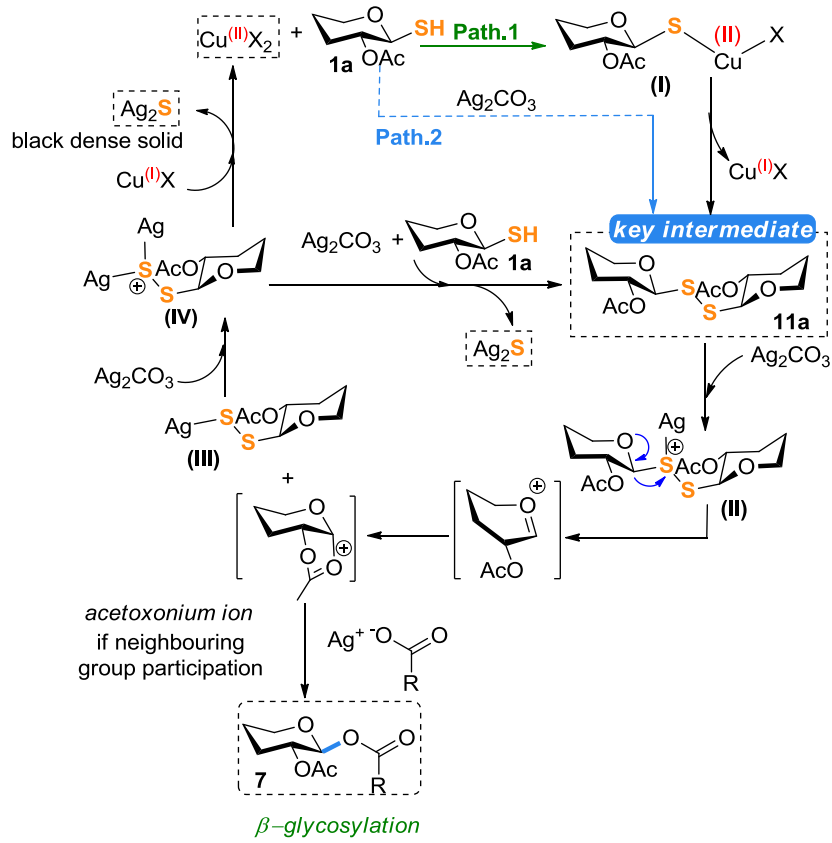

the reaction of $\mathbf{2 a}$ was performed with the disulfide 11a in the presence of only $\mathrm{Ag}_{2} \mathrm{CO}_{3}$ (yield of $7 \mathbf{a}=68 \%$ ), thus suggesting that this path may be involved in the mechanism. However, when performed with a catalytic amount $(10 \mathrm{~mol} \%)$ of the disulfide $11 \mathbf{a}$ and 1.5 equiv. of $\mathbf{1 a}$ in the presence of $\mathbf{2 a}$ and $\mathrm{Ag}_{2} \mathrm{CO}_{3}$, without adding the $\mathrm{Cu}$-catalyst (Scheme 4, eq. 7), the reaction proceeded with only $53 \%$ yield of $7 \mathbf{a}$ (see supporting information). The improvement observed in the presence of copper salt led us to propose an alternative path for the evolution of $\mathbf{I V}$. The latter can react with $\mathrm{Cu}(\mathrm{I})$ to release $\mathrm{Ag}_{2} \mathrm{~S}$ regenerating the $\mathrm{Cu}(\mathrm{II})$ catalyst.

In conclusion, we reported an unconventional thiol- to-acid two nucleophiles coupling method for the diastereoselective synthesis of 1,2-trans-O-glycosylated esters through a $\mathrm{Cu}^{\text {(II) }}$ - or $\mathrm{Co}^{\text {(II) }}$-catalyzed activation of glycosyl thiols. This approach tolerates a wide range of functional groups, offers a unique strategy to access 1,2-trans- $O$-glycosylated esters, and streamlines synthesis of pharmaceutically important compounds. Furthermore, efforts to expand the scope of this transformation are undergoing.

\section{EXPERIMENTAL SECTION}

\section{General Procedure}

The coupling products were all identified by usual physical methods, e.g., ${ }^{1} \mathrm{H}$ NMR, ${ }^{13} \mathrm{C}$ NMR, IR, MS. ${ }^{1} \mathrm{H}$ and ${ }^{13} \mathrm{C} \mathrm{NMR}$ spectra were measured in $\mathrm{CDCl}_{3}$, with a Bruker Avance-300. ${ }^{1} \mathrm{H}$ chemical shifts are reported in ppm from an internal standard TMS or of residual chloroform (7.26 ppm). The following abbreviations are used: $\mathrm{m}$ (multiplet), s (singlet), bs (broad singlet), d (doublet), t (triplet), dd (doublet of doublet), td (triplet of doublet), q (quadruplet), qui (quintuplet), sex (sextuplet). ${ }^{13} \mathrm{C}$ chemical shift are reported in ppm from central peak of deuteriochloroform (77.14). IR spectra were measured on a Bruker Vector 22 spectrophotometer and are reported in wave numbers $\left(\mathrm{cm}^{-1}\right)$. MS were recorded on a Micromass spectrometer. Analytical TLC was performed on Merck precoated silica gel 60F plates. Merck silica gel $60(0.015-$ $0.040 \mathrm{~mm}$ ) was used for column chromatography. Melting points were recorded on a Büchi B-450 apparatus (accuracy: up to $100{ }^{\circ} \mathrm{C}\left( \pm 0.3{ }^{\circ} \mathrm{C}\right)$; up to $250{ }^{\circ} \mathrm{C}\left( \pm 0.5^{\circ} \mathrm{C}\right)$; up to $400{ }^{\circ} \mathrm{C}$ 
$\left( \pm 0.8^{\circ} \mathrm{C}\right)$. High resolution mass spectra (HR-MS) were recorded on a Bruker MicroTOF spectrometer, using ESI or APCI with methanol as the carrier solvent. Biotage - microwave reaction vial (2-5 $\mathrm{mL}$, ref.351521) and (10-5 mL, ref.354833) for large scale were used. Nominal and exact $m / z$ values are reported in Daltons. Unless otherwise noted, other materials are obtained from commercial suppliers were used without further purification. Solvents used were used as received without further purification. Thiosugars were prepared following the literature procedure. ${ }^{25,31}$

\section{General Procedure (A) for Cu-Catalyzed glycosylation with 1-thiosugars}

A flame-dried microwave reaction vial $(2-5 \mathrm{~mL})$ was charged with $\mathrm{Cu}(\mathrm{acac})_{2}(5 \mathrm{~mol} \%), \mathrm{Ag}_{2} \mathrm{CO}_{3}$ (0.53 mmol, 3.0 equiv.), commercial acid carboxylic ( $0.19 \mathrm{mmol}, 1.0$ equiv.) and the thiosugar $(0.29 \mathrm{mmol}, 1.5$ equiv. $)$. Then, the reaction mixture was flushed under Argon for 10 minutes. Trifluorotoluene $(2 \mathrm{~mL})$ was added and the tube was sealed with a Teflon screwcap, and the mixture was stirred at $130{ }^{\circ} \mathrm{C}$ under microwave irradiation until completion. The resulting suspension was cooled to room temperature. The reaction mixture was then filtered through a small pad of Celite and rinsed three times with DCM:MeOH 9:1 (3x10 mL). After concentration under reduced pressure, the residue was purified by silica gel column chromatography to afford the desired product.

\subsection{General Procedure (B) for Co-Catalyzed glycosylation with 1-thiosugars}

A flame-dried sealed tube was charged with $\mathrm{Co}(\mathrm{acac})_{2}(10$ mol\%), $\mathrm{Ag}_{2} \mathrm{CO}_{3}$ (0.53 mmol, 3.0 equiv.), commercial acid carboxylic (0.19 mmol, 1.0 equiv.) and the thiosugar $(0.29$ mmol, 1.5 equiv.).Then, the reaction mixture was flushed under Argon for 10 minutes. Trifluorotoluene $(2 \mathrm{~mL})$ was added and the tube was sealed with a Teflon screwcap, and the mixture was stirred at $150{ }^{\circ} \mathrm{C}$ until completion. The resulting suspension was cooled to room temperature. The reaction mixture was then filtered through a small pad of Celite and rinsed three times with EtOAc $(3 \times 10 \mathrm{~mL})$. After concentration under reduced pressure, the residue was purified by silica gel column chromatography to afford the desired product.

\section{NMR Data for the glycosyl esters 7a-z, 7aa, 7ab, 7ac, 8af}

\section{$\beta$-D-Glucopyranose, 2,3,4,6-tetraacetate 1-benzoate [7a]} Compound 7a was prepared by using both general procedures (A) (reaction time: $15 \mathrm{~min}$ ), and (B) (reaction time: $1 \mathrm{~h}$ ), with commercial benzoic acid 5a (23.4 $\mathrm{mg}, 0.19 \mathrm{mmol}, 1.0$ equiv.) and $1 \mathrm{a}$ (104.9 mg, $0.29 \mathrm{mmol}, 1.5$ equiv.), which afforded the product as a white solid (with $\mathrm{Cu}$-catalyst $0.15 \mathrm{mmol}, 79 \%$ yield, with Co-catalyst $0.142 \mathrm{mmol}, 77 \%$ yield) after column chromatography EtOAc/Cyclohexane 3:7);

1 mmol scale protocol: A flame-dried microwave reaction vial (10-20 mL) was charged with $\mathrm{Cu}(\mathrm{acac})_{2}(5 \mathrm{~mol} \%)$, $\mathrm{Ag}_{2} \mathrm{CO}_{3}$ (3 mmol, 3.0 equiv.), commercial acid carboxylic (1 mmol, 1.0 equiv.) and the thiosugar (1.5 mmol, 1.5 equiv.).Then, the reaction mixture was flushed under Argon for 10 minutes. Trifluorotoluene $(10 \mathrm{~mL})$ was added and the tube was sealed with a Teflon screwcap, and the mixture was stirred at $130{ }^{\circ} \mathrm{C}$ under microwave irradiation until completion. The resulting suspension was cooled to room tempera- ture. The reaction mixture was then filtered through a small pad of Celite and rinsed three times with DCM:MeOH 9:1 $(3 \times 10 \mathrm{~mL})$. After concentration under reduced pressure, the residue was purified by silica gel column chromatography to afford the desired product $7 \mathbf{a}$ in $75 \%$ yield. $\mathbf{T L C} \mathbf{R}_{\mathbf{f}}=0.54$ (EtOAc/Cyclohexane, 1:1, $\left.\mathrm{SiO}_{2}\right)$; mp: $132.3-134.3{ }^{\circ} \mathrm{C}$; $[\boldsymbol{\alpha}]_{\mathbf{D}}{ }^{19}=-16.2\left(\mathrm{c}, 1.00\right.$ in $\left.\mathrm{CHCl}_{3}\right)$; IR (neat, $\left.\mathbf{~ c m}^{-1}\right)$ 2964, 2905, 1759, 1367, 1243, 1086, 1066, 1033; ${ }^{\mathbf{1}} \mathbf{H}$ NMR (300 MHz, $\left.\mathbf{C D C l}_{3}\right) \boldsymbol{\delta}(\mathbf{p p m}) 8.03(\mathrm{~d}, J=7.6 \mathrm{~Hz}, 2 \mathrm{H}), 7.60(\mathrm{t}, J=7.3 \mathrm{~Hz}$, $1 \mathrm{H}), 7.45(\mathrm{t}, J=7.7 \mathrm{~Hz}, 2 \mathrm{H}), 5.92(\mathrm{dd}, J=5.6,2.5 \mathrm{~Hz}, 1 \mathrm{H})$, $5.34(\mathrm{dd}, J=5.7,3.1 \mathrm{~Hz}, 2 \mathrm{H}), 5.25-5.10(\mathrm{~m}, 1 \mathrm{H}), 4.32(\mathrm{dd}, J$ $=12.5,4.4 \mathrm{~Hz}, 1 \mathrm{H}), 4.13(\mathrm{dd}, J=12.6,2.3 \mathrm{~Hz}, 1 \mathrm{H}), 3.94$ (ddd, $J=9.9,4.5,2.3 \mathrm{~Hz}, 1 \mathrm{H}), 2.06(\mathrm{~s}, 3 \mathrm{H}), 2.04(\mathrm{~s}, 3 \mathrm{H}), 2.03(\mathrm{~s}$, 3H), $1.98(\mathrm{~s}, 3 \mathrm{H}) ;{ }^{13} \mathbf{C}\{\mathbf{1 H}\}$ NMR (75 MHz, $\left.\mathbf{C D C l}_{3}\right) \boldsymbol{\delta}$ (ppm) $170.7\left(\mathrm{C}_{\mathrm{q}}\right), 170.2\left(\mathrm{C}_{\mathrm{q}}\right), 169.5\left(\mathrm{C}_{\mathrm{q}}\right), 169.4\left(\mathrm{C}_{\mathrm{q}}\right), 164.6\left(\mathrm{C}_{\mathrm{q}}\right)$, $134.1(\mathrm{CH}), 130.3(2 \times \mathrm{CH}), 128.7(2 \times \mathrm{CH}), 128.5\left(\mathrm{C}_{\mathrm{q}}\right), 92.4$ $(\mathrm{CH}), 72.9(\mathrm{CH}), 72.8(\mathrm{CH}), 70.3(\mathrm{CH}), 68.0(\mathrm{CH}), 61.6$ $\left(\mathrm{CH}_{2}\right), 20.8\left(\mathrm{CH}_{3}\right), 20.7\left(3 \times \mathrm{CH}_{3}\right) ;$ HRMS (ESI) $(\mathbf{M}+\mathbf{~ N a})^{+}$ $\mathrm{m} / \mathrm{z}$ calculated for $\mathrm{C}_{21} \mathrm{H}_{24} \mathrm{O}_{11} \mathrm{Na} 475.1211$, found 475.1198 .

\section{$\beta$-D-Glucopyranose, $\quad$ 2,3,4,6-tetraacetate $\quad 1-(2$} methoxybenzoate) [7b]

Compound $\mathbf{7 b}$ was prepared by using both general procedures (A) (reaction time: $15 \mathrm{~min}$ ), and (B) (reaction time: $1 \mathrm{~h}$ ), with commercial 2-methoxybenzoic acid $\mathbf{5 b}(29.2 \mathrm{mg}, 0.19 \mathrm{mmol}$, 1.0 equiv.) and $1 \mathrm{a}$ (104.9 mg, $0.29 \mathrm{mmol}, 1.5$ equiv.), which afforded the product as a white solid (with $\mathrm{Cu}$-catalyst 0.133 mmol, $70 \%$ yield, with Co-catalyst $0.0836 \mathrm{mmol}, 44 \%$ yield) after column chromatography EtOAc/Cyclohexane 3:7): TLC $\mathbf{R}_{\mathbf{f}}=0.36\left(\right.$ EtOAc/Cyclohexane, $\left.1: 1, \mathrm{SiO}_{2}\right) ; \mathbf{m p}: 91.9-93.0$ ${ }^{\circ} \mathrm{C} ;[\boldsymbol{\alpha}]_{\mathbf{D}}{ }^{19}=-12.7$ (c, 1.00 in $\mathrm{CHCl}_{3}$ ); IR (neat, $\mathbf{c m}^{-1}$ ) 2974, 2920, 1758, 1601, 1492, 1366, 1206, 1079, 1037; ${ }^{1} \mathbf{H}$ NMR (300 MHz, CDCl $\mathbf{~}_{3} \boldsymbol{\delta}$ (ppm) $7.86(\mathrm{~d}, J=8.2 \mathrm{~Hz}, 1 \mathrm{H}), 7.52(\mathrm{t}$, $J=7.9 \mathrm{~Hz}, 1 \mathrm{H}), 6.99(\mathrm{t}, J=7.2 \mathrm{~Hz}, 2 \mathrm{H}), 5.95(\mathrm{~d}, J=8.0 \mathrm{~Hz}$, $1 \mathrm{H}), 5.32(\mathrm{dd}, J=5.4,3.3 \mathrm{~Hz}, 2 \mathrm{H}), 5.23-5.14(\mathrm{~m}, 1 \mathrm{H}), 4.31$ (dd, $J=12.5,4.4 \mathrm{~Hz}, 1 \mathrm{H}), 4.12(\mathrm{dd}, J=12.5,2.1 \mathrm{~Hz}, 1 \mathrm{H})$, $3.95-3.91(\mathrm{~m}, 1 \mathrm{H}), 3.90$ (s, 3H), 2.06 (s, 3H), $2.04(\mathrm{~s}, 3 \mathrm{H})$, $2.03(\mathrm{~s}, 3 \mathrm{H}), 2.00(\mathrm{~s}, 3 \mathrm{H}) ;{ }^{13} \mathbf{C}\{\mathbf{1 H}\} \mathbf{N M R}\left(\mathbf{7 5} \mathbf{~ M H z}, \mathbf{C D C l}_{3}\right) \boldsymbol{\delta}$ (ppm) $170.8\left(\mathrm{C}_{\mathrm{q}}\right), 170.3\left(\mathrm{C}_{\mathrm{q}}\right), 169.6\left(\mathrm{C}_{\mathrm{q}}\right), 169.5\left(\mathrm{C}_{\mathrm{q}}\right), 163.5$ $\left(\mathrm{C}_{\mathrm{q}}\right), 160.4\left(\mathrm{C}_{\mathrm{q}}\right), 135.1(\mathrm{CH}), 132.7(\mathrm{CH}), 120.4(\mathrm{CH}), 117.6$ $\left(\mathrm{C}_{\mathrm{q}}\right), 112.2(\mathrm{CH}), 92.0(\mathrm{CH}), 73.0(\mathrm{CH}), 72.9(\mathrm{CH}), 70.4(\mathrm{CH})$, $68.1(\mathrm{CH}), 61.7\left(\mathrm{CH}_{2}\right), 56.0\left(\mathrm{CH}_{3}\right), 20.8\left(\mathrm{CH}_{3}\right), 20.7(3 \mathrm{x}$ $\left.\mathrm{CH}_{3}\right)$; HRMS (ESI) (M + Na) ${ }^{+} \mathrm{m} / \mathrm{z}$ calculated for $\mathrm{C}_{22} \mathrm{H}_{26} \mathrm{O}_{12} \mathrm{Na}$ 505.1322, found 505.1324.

\section{$\beta$-D-Glucopyranose, \\ 2,3,4,6-tetraacetate \\ $1-(2,6-$} dimethoxybenzoate) [7c]

Compound 7c was prepared by using both general procedures (A) (reaction time: $15 \mathrm{~min}$ ), and (B) (reaction time: $1 \mathrm{~h}$ ), with commercial 2,6-dimethoxybenzoic acid 5c $(35.0 \mathrm{mg}, 0.19$ mmol, 1.0 equiv.) and 1 a (104.9 mg, $0.29 \mathrm{mmol}, 1.5$ equiv.), which afforded the product as a white solid (with $\mathrm{Cu}$-catalyst $0.185 \mathrm{mmol}, 70 \%$ yield, with Co-catalyst $0.0855 \mathrm{mmol}, 45 \%$ yield) after column chromatography EtOAc/Cyclohexane 3:7): TLC $\mathbf{R}_{\mathbf{f}}=0.32\left(\right.$ EtOAc/Cyclohexane, $\left.1: 1, \mathrm{SiO}_{2}\right)$; mp: $185.7-$ $187.2{ }^{\circ} \mathrm{C} ;[\boldsymbol{\alpha}]_{\mathbf{D}}{ }^{\mathbf{2 0}}=-4.7\left(\mathrm{c}, 1.00\right.$ in $\left.\mathrm{CHCl}_{3}\right)$; IR (neat, $\mathbf{c m}^{-\mathbf{1}}$ ) 2954, 2841, 1759, 1597, 1477, 1366, 1258, 1235, 1133, 1024; ${ }^{1} \mathbf{H}$ NMR (300 MHz, $\left.\mathbf{C D C l}_{3}\right) \boldsymbol{\delta}$ (ppm) $7.30(\mathrm{~d}, J=8.4 \mathrm{~Hz}$, $1 \mathrm{H}), 6.54(\mathrm{~s}, 1 \mathrm{H}), 6.52(\mathrm{~s}, 1 \mathrm{H}), 6.07(\mathrm{~d}, J=8.2 \mathrm{~Hz}, 1 \mathrm{H}), 5.34-$ $5.25(\mathrm{~m}, 1 \mathrm{H}), 5.23-5.13(\mathrm{~m}, 2 \mathrm{H}), 4.33(\mathrm{dd}, J=12.4,4.5 \mathrm{~Hz}$, $1 \mathrm{H}), 4.19-4.10(\mathrm{~m}, 1 \mathrm{H}), 3.90$ (ddd, $J=9.9,4.6,2.4 \mathrm{~Hz}, 1 \mathrm{H})$, $3.78(\mathrm{~s}, 6 \mathrm{H}), 2.07$ (s, 3H), $2.05(\mathrm{~s}, 3 \mathrm{H}), 2.03$ (s, 3H), $2.01(\mathrm{~s}$, 
$3 \mathrm{H}) ;{ }^{13} \mathbf{C}\{\mathbf{1 H}\}$ NMR $\left(\mathbf{7 5} \mathbf{~ M H z}, \mathbf{C D C l}_{\mathbf{3}}\right) \boldsymbol{\delta}(\mathbf{p p m}) 170.8\left(\mathrm{C}_{\mathrm{q}}\right)$, $170.3\left(\mathrm{C}_{\mathrm{q}}\right), 169.5\left(\mathrm{C}_{\mathrm{q}}\right), 169.3\left(\mathrm{C}_{\mathrm{q}}\right), 164.3\left(\mathrm{C}_{\mathrm{q}}\right), 157.9\left(2 \times \mathrm{C}_{\mathrm{q}}\right)$, $132.1(\mathrm{CH}), 111.3\left(\mathrm{C}_{\mathrm{q}}\right), 104.1(2 \times \mathrm{CH}), 92.2(\mathrm{CH}), 73.2(\mathrm{CH})$, $73.1(\mathrm{CH}), 70.7(\mathrm{CH}), 68.0(\mathrm{CH}), 61.8\left(\mathrm{CH}_{2}\right), 56.1\left(2 \times \mathrm{CH}_{3}\right)$, $20.8\left(\mathrm{CH}_{3}\right), 20.7\left(3 \times \mathrm{CH}_{3}\right) ; \mathbf{H R M S}($ ESI $)(\mathbf{M}+\mathbf{N a})^{+} \mathrm{m} / \mathrm{z}$ calculated for $\mathrm{C}_{23} \mathrm{H}_{28} \mathrm{O}_{13} \mathrm{Na} 535.1428$, found 535.1431.

\section{$\beta$-D-Glucopyranose, $\quad$ 2,3,4,6-tetraacetate $\quad 1-(2,5-$} dimethoxybenzoate) [7d]

Compound 7d was prepared by using both general procedures (A) (reaction time: $15 \mathrm{~min}$ ), and (B) (reaction time: 1h), with commercial 2,5-dimethoxybenzoic acid 5d $(35.0 \mathrm{mg}, 0.19$ mmol, 1.0 equiv.) and $\mathbf{1 a}(104.9 \mathrm{mg}, 0.29 \mathrm{mmol}, 1.5$ equiv.), which afforded the product as a white solid (with $\mathrm{Cu}$-catalyst $0.138 \mathrm{mmol}, 70 \%$ yield, with Co-catalyst $0.060 \mathrm{mmol}, 32 \%$ yield) after column chromatography EtOAc/Cyclohexane 3:7): TLC $\mathbf{R}_{\mathbf{f}}=0.25\left(\right.$ EtOAc/Cyclohexane, $\left.1: 1, \mathrm{SiO}_{2}\right)$; mp: $126.0-$ $127.2{ }^{\circ} \mathrm{C} ;[\boldsymbol{\alpha}]_{\mathbf{D}}{ }^{20}=-2.4\left(\mathrm{c}, 1.00\right.$ in $\left.\mathrm{CHCl}_{3}\right) ;$ IR $\left(\right.$ neat, $\left.\mathbf{~ c m}^{-1}\right)$ 2964, 2929, 1757, 1502, 1367, 1238, 1080, 1039; ${ }^{1} \mathbf{H}$ NMR $\left(300 \mathbf{M H z}, \mathbf{C D C l}_{3}\right) \boldsymbol{\delta}$ (ppm) $7.39(\mathrm{~d}, J=3.2 \mathrm{~Hz}, 1 \mathrm{H}), 7.08$ (dd, $J=9.1,3.2 \mathrm{~Hz}, 1 \mathrm{H}), 6.92(\mathrm{~d}, J=9.1 \mathrm{~Hz}, 1 \mathrm{H}), 5.93$ (dd, $J$ $=5.2,2.9 \mathrm{~Hz}, 1 \mathrm{H}), 5.32(\mathrm{dd}, J=5.6,3.2 \mathrm{~Hz}, 2 \mathrm{H}), 5.24-5.14$ $(\mathrm{m}, 1 \mathrm{H}), 4.32(\mathrm{dd}, J=12.5,4.5 \mathrm{~Hz}, 1 \mathrm{H}), 4.13(\mathrm{dd}, J=12.5$, $2.4 \mathrm{~Hz}, 1 \mathrm{H}), 3.92(\mathrm{ddd}, J=10.1,4.4,2.1 \mathrm{~Hz}, 1 \mathrm{H}), 3.86(\mathrm{~s}$, $3 \mathrm{H}), 3.80$ (s, 3H), 2.07 (s, 3H), 2.04 (s, 3H), 2.03 (s, 3H), 2.01 $(\mathrm{s}, 3 \mathrm{H}) ;{ }^{13} \mathbf{C}\{\mathbf{1 H}\}$ NMR $\left(\mathbf{7 5} \mathbf{~ M H z}, \mathbf{C D C l}_{3}\right) \boldsymbol{\delta}(\mathbf{p p m}) 170.8\left(\mathrm{C}_{\mathrm{q}}\right)$, $170.2\left(\mathrm{C}_{\mathrm{q}}\right), 169.6\left(\mathrm{C}_{\mathrm{q}}\right), 169.5\left(\mathrm{C}_{\mathrm{q}}\right), 163.2\left(\mathrm{C}_{\mathrm{q}}\right), 154.9\left(\mathrm{C}_{\mathrm{q}}\right)$, $153.1\left(\mathrm{C}_{\mathrm{q}}\right), 121.9(\mathrm{CH}), 117.8\left(\mathrm{C}_{\mathrm{q}}\right), 116.1(\mathrm{CH}), 113.9(\mathrm{CH})$, $92.1(\mathrm{CH}), 72.9(2 \times \mathrm{CH}), 70.4(\mathrm{CH}), 68.1(\mathrm{CH}), 61.7\left(\mathrm{CH}_{2}\right)$, $56.6\left(\mathrm{CH}_{3}\right), 56.0\left(\mathrm{CH}_{3}\right), 20.9\left(\mathrm{CH}_{3}\right), 20.8\left(3 \times \mathrm{CH}_{3}\right)$; HRMS (ESI) $(\mathbf{M}+\mathbf{N a})^{+} \mathrm{m} / z$ calculated for $\mathrm{C}_{23} \mathrm{H}_{28} \mathrm{O}_{13} \mathrm{Na} 535.1428$, found 535.1434 .

\section{$\beta$-D-Glucopyranose, $\quad 2,3,4,6$-tetraacetate $\quad 1-(3,4,5-$ trimethoxybenzoate) [7e]}

Compound 7e was prepared by using both general procedures (A) (reaction time: $15 \mathrm{~min}$ ), and (B) (reaction time: $1 \mathrm{~h}$ ), with commercial 3,4,5-trimethoxybenzoic acid 5e $(40.8 \mathrm{mg}$, $0.19 \mathrm{mmol}, 1.0$ equiv.) and $1 \mathrm{1a}(104.9 \mathrm{mg}, 0.29 \mathrm{mmol}, 1.5$ equiv.), which afforded the product as a white solid (with $\mathrm{Cu}$ catalyst $0.100 \mathrm{mmol}, 53 \%$ yield, with Co-catalyst $0.119 \mathrm{mmol}$, $67 \%$ yield) after column chromatography EtOAc/Cyclohexane 3:7): $\mathbf{T L C} \mathbf{R}_{\mathbf{f}}=0.40\left(\right.$ EtOAc/Cyclohexane, 1:1, $\left.\mathrm{SiO}_{2}\right)$; $\mathbf{m p}$ : $89.7-90.7{ }^{\circ} \mathrm{C} ;[\boldsymbol{\alpha}]_{\mathbf{D}}{ }^{20}=-26.1\left(\mathrm{c}, 1.00\right.$ in $\left.\mathrm{CHCl}_{3}\right) ; \mathbf{I R}$ (neat, $\mathbf{c m}^{-1}$ ) 3496, 2949, 2841, 1757, 1590, 1504, 1336, 1206, 1126, 1031; ${ }^{1} \mathbf{H}$ NMR (300 MHz, $\left.\mathbf{C D C l}_{3}\right) \boldsymbol{\delta}$ (ppm) 7.29 (s, 2H), $5.85(\mathrm{dd}, J=5.6,2.4 \mathrm{~Hz}, 1 \mathrm{H}), 5.37-5.30(\mathrm{~m}, 2 \mathrm{H}), 5.26-5.12$ (m, 1H), 4.34 (dd, $J=12.5,4.4 \mathrm{~Hz}, 1 \mathrm{H}), 4.17-4.08(\mathrm{~m}, 1 \mathrm{H})$, $3.99-3.91(\mathrm{~m}, 1 \mathrm{H}), 3.90(\mathrm{~s}, 3 \mathrm{H}), 3.89(\mathrm{~s}, 6 \mathrm{H}), 2.06(\mathrm{~s}, 3 \mathrm{H})$, $2.04(\mathrm{~s}, 3 \mathrm{H}), 2.03(\mathrm{~s}, 3 \mathrm{H}), 1.98(\mathrm{~s}, 3 \mathrm{H}) ;{ }^{13} \mathbf{C}\{\mathbf{1 H}\}$ NMR $(\mathbf{7 5}$ MHz, $\left.\mathbf{C D C l}_{3}\right) \boldsymbol{\delta}$ (ppm) $170.7\left(\mathrm{C}_{\mathrm{q}}\right), 170.1\left(\mathrm{C}_{\mathrm{q}}\right), 169.6\left(\mathrm{C}_{\mathrm{q}}\right)$, $169.5\left(\mathrm{C}_{\mathrm{q}}\right), 164.2\left(\mathrm{C}_{\mathrm{q}}\right), 153.2\left(2 \times \mathrm{C}_{\mathrm{q}}\right), 143.2\left(\mathrm{C}_{\mathrm{q}}\right), 123.3\left(\mathrm{C}_{\mathrm{q}}\right)$, $107.5(2 \times \mathrm{CH}), 92.6(\mathrm{CH}), 72.9(\mathrm{CH}), 72.6(\mathrm{CH}), 70.3(\mathrm{CH})$, $68.1(\mathrm{CH}), 61.6\left(\mathrm{CH}_{2}\right), 61.1\left(\mathrm{CH}_{3}\right), 56.4\left(2 \times \mathrm{CH}_{3}\right), 20.8$ $\left(\mathrm{CH}_{3}\right), 20.7\left(3 \times \mathrm{CH}_{3}\right) ;$ HRMS (ESI) $(\mathbf{M}+\mathbf{N a})^{+} \mathrm{m} / z$ calculated for $\mathrm{C}_{24} \mathrm{H}_{30} \mathrm{O}_{14} \mathrm{Na} 565.1528$, found 565.1534.

\section{$\beta$-D-Glucopyranose, $\quad$ 2,3,4,6-tetraacetate $\quad 1-(2,4,6$ -} trimethylbenzoate) $[\mathbf{7 f}]$

Compound 7f was prepared by using the general procedure (B) (reaction time: 1h), with commercial 2,4,6-trimethylbenzoic acid $\mathbf{5 f}(31.5 \mathrm{mg}, 0.19 \mathrm{mmol}, 1.0$ equiv.) and $1 \mathrm{a}$ (104.9 mg, $0.29 \mathrm{mmol}, 1.5$ equiv.), which af- forded the product as a white solid $(61.2 \mathrm{mg}, 65 \%$ yield after column chromatography EtOAc/Cyclohexane 3:7); TLC $\mathbf{R}_{\mathbf{f}}=$ 0.60 (EtOAc/Cyclohexane, $1: 1, \mathrm{SiO}_{2}$ ); mp: $136.2-137.5^{\circ} \mathrm{C}$; $\left[\mathbf{\alpha}_{\mathbf{D}}{ }^{\mathbf{2 0}}=-8.2\left(\mathrm{c}, 1.00\right.\right.$ in $\left.\mathrm{CHCl}_{3}\right) ;$ IR (neat, $\mathbf{~ c m}^{-1}$ ) 2969, 2920, 1757, 1612, 1366, 1239, 1167, 1060, 1036; ${ }^{1}$ H NMR (300 MHz, $\left.\mathbf{C D C l}_{3}\right) \boldsymbol{\delta}(\mathbf{p p m}) 6.84(\mathrm{~s}, 2 \mathrm{H}), 5.99(\mathrm{~d}, J=8.1 \mathrm{~Hz}, 1 \mathrm{H})$, $5.34-5.25(\mathrm{~m}, 1 \mathrm{H}), 5.18(\mathrm{dd}, J=20.4,9.0 \mathrm{~Hz}, 2 \mathrm{H}), 4.32(\mathrm{dd}$, $J=12.4,4.7 \mathrm{~Hz}, 1 \mathrm{H}), 4.19-4.11(\mathrm{~m}, 1 \mathrm{H}), 3.95-3.87(\mathrm{~m}$, $1 \mathrm{H}), 2.27$ (s, 3H), 2.26 (s, 6H), 2.07 (s, 3H), 2.04 (s, 3H), 2.02 $(\mathrm{s}, 3 \mathrm{H}), 2.01(\mathrm{~s}, 3 \mathrm{H}) ;{ }^{13} \mathbf{C}\{\mathbf{1 H}\}$ NMR $\left(\mathbf{7 5} \mathbf{~ M H z}, \mathbf{C D C l}_{3}\right) \delta$ (ppm) $170.7\left(\mathrm{C}_{\mathrm{q}}\right), 170.3\left(\mathrm{C}_{\mathrm{q}}\right), 169.5\left(\mathrm{C}_{\mathrm{q}}\right), 169.2\left(\mathrm{C}_{\mathrm{q}}\right), 168.1$ $\left(\mathrm{C}_{\mathrm{q}}\right), 140.3\left(\mathrm{C}_{\mathrm{q}}\right), 135.7\left(2 \times \mathrm{C}_{\mathrm{q}}\right), 129.1\left(\mathrm{C}_{\mathrm{q}}\right), 128.7(2 \times \mathrm{CH})$, $91.9(\mathrm{CH}), 73.3(\mathrm{CH}), 72.9(\mathrm{CH}), 70.3(\mathrm{CH}), 68.1(\mathrm{CH}), 61.7$ $\left(\mathrm{CH}_{2}\right), 21.3\left(\mathrm{CH}_{3}\right), 20.8\left(\mathrm{CH}_{3}\right), 20.7\left(3 \times \mathrm{CH}_{3}\right), 19.9\left(2 \times \mathrm{CH}_{3}\right)$; HRMS (ESI) $(\mathbf{M}+\mathbf{N a})^{+} \mathrm{m} / z$ calculated for $\mathrm{C}_{24} \mathrm{H}_{30} \mathrm{O}_{11} \mathrm{Na}$ 517.1686, found 517.1678; Elemental Analysis calculated: C, $58.29 \%$;, $6.12 \%$; O, $35.59 \%$, found: C, $58.07 \%$;, 6.17 $\% ; \mathrm{O}, 35.03 \%$.

\section{$\beta$-D-Glucopyranose, 2,3,4,6-tetraacetate 1-(4-aminobenzoate)} $[7 \mathrm{~g}]$

Compound 7g was prepared by using both general procedures (A) (reaction time: $15 \mathrm{~min}$ ), and (B) (reaction time: 1h), with commercial 4-aminolbenzoic acid $\mathbf{5 g}(26.3 \mathrm{mg}, 0.19$ mmol, 1.0 equiv.) and $1 \mathrm{a}$ (104.9 mg, $0.29 \mathrm{mmol}, 1.5$ equiv.), which afforded the product as a white solid (with $\mathrm{Cu}$-catalyst $0.145 \mathrm{mmol}$, 50\% yield, with Co-catalyst $0.1 \mathrm{mmol}, 53 \%$ yield) after column chromatography EtOAc/Cyclohexane 3:7): TLC $\mathbf{R}_{\mathbf{f}}=0.28\left(\right.$ EtOAc/Cyclohexane, $\left.1: 1, \mathrm{SiO}_{2}\right)$; mp: $138.2-$ $140.4{ }^{\circ} \mathrm{C} ;[\boldsymbol{\alpha}]_{\mathbf{D}}{ }^{19}=-5.6\left(\mathrm{c}, 1.00\right.$ in $\left.\mathrm{CHCl}_{3}\right)$; IR (neat, $\mathbf{~ c m}^{-\mathbf{1}}$ ) 3487, 3384, 2964, 2924, 1758, 1602, 1366, 1210, 1065, 1037; ${ }^{1} \mathbf{H}$ NMR (300 MHz, $\left.\mathbf{C D C l}_{3}\right) \boldsymbol{\delta}$ (ppm) $7.84(\mathrm{~d}, J=8.7 \mathrm{~Hz}$, $2 \mathrm{H}), 6.63(\mathrm{~d}, J=8.7 \mathrm{~Hz}, 2 \mathrm{H}), 5.88(\mathrm{dd}, J=6.0,2.4 \mathrm{~Hz}, 1 \mathrm{H})$, $5.36-5.27(\mathrm{~m}, 2 \mathrm{H}), 5.23-5.12(\mathrm{~m}, 1 \mathrm{H}), 4.32(\mathrm{dd}, J=12.7$, $4.2 \mathrm{~Hz}, 1 \mathrm{H}), 4.14$ (d, $J=2.9 \mathrm{~Hz}, 1 \mathrm{H}), 3.92$ (ddd, $J=10.3,4.4$, $2.2 \mathrm{~Hz}, 1 \mathrm{H}), 2.07$ (s, 3H), 2.04 (s, 3H), 2.04 (s, 3H), 1.98 (s, $3 \mathrm{H})$, no $\mathrm{NH}_{2}$ protons observed; ${ }^{13} \mathbf{C}\{1 \mathbf{H}\}$ NMR (75 MHz, $\left.\mathbf{C D C l}_{3}\right) \boldsymbol{\delta}(\mathbf{p p m}) 170.8\left(\mathrm{C}_{\mathrm{q}}\right), 170.2\left(\mathrm{C}_{\mathrm{q}}\right), 169.6\left(2 \times \mathrm{C}_{\mathrm{q}}\right), 164.5$ $\left(\mathrm{C}_{\mathrm{q}}\right), 151.9\left(\mathrm{C}_{\mathrm{q}}\right), 132.6(2 \times \mathrm{CH}), 117.6\left(\mathrm{C}_{\mathrm{q}}\right), 114.0(2 \times \mathrm{CH})$, $92.1(\mathrm{CH}), 72.9(\mathrm{CH}), 72.8(\mathrm{CH}), 70.4(\mathrm{CH}), 68.1(\mathrm{CH}), 61.7$ $\left(\mathrm{CH}_{2}\right), 20.8\left(\mathrm{CH}_{3}\right), 20.7\left(3 \times \mathrm{CH}_{3}\right)$; HRMS (ESI) $(\mathbf{M}+\mathbf{N a})^{+}$ $m / z$ calculated for $\mathrm{C}_{21} \mathrm{H}_{25} \mathrm{NO}_{11} \mathrm{Na} 490.1325$, found 490.1327 .

\section{$\beta$-D-Glucopyranose, 2,3,4,6-tetraacetate $\quad$ 1-(4-} hydroxybenzoate) [7h]

Compound $\mathbf{7 h}$ was prepared by using the general procedure (B) (reaction time: 1h), with commercial 4-hydroxylbenzoic acid $\mathbf{5 h}$ (26.5 mg, 0.19 mmol, 1.0 equiv.) and $\mathbf{1 a}^{[25]}$ (104.9 mg, 0.29 mmol, 1.5 equiv.), which afforded the product as a white solid (37.2 mg, $41 \%$ yield after column chromatography EtOAc/Cyclohexane 3:7); TLC $\quad \mathbf{R}_{\mathbf{f}}=0.28$ (EtOAc/Cyclohexane, 1:1, $\mathrm{SiO}_{2}$ ); mp: 178.3 - $179.6{ }^{\circ} \mathrm{C}$; $[\boldsymbol{\alpha}]_{\mathbf{D}}{ }^{19}=-7.3\left(\mathrm{c}, 1.00\right.$ in $\left.\mathrm{CHCl}_{3}\right)$; IR (neat, $\mathbf{~ c m}^{-1}$ ) 3418, 2969, 2910, 1757, 1609, 1367, 1212, 1067, 1038; ${ }^{\mathbf{1}} \mathbf{H}$ NMR (300 MHz, $\left.\mathbf{C D C l}_{3}\right) \boldsymbol{\delta}$ (ppm) $7.93(\mathrm{dd}, J=7.0,4.8 \mathrm{~Hz}, 2 \mathrm{H}), 6.85$ $(\mathrm{dd}, J=7.0,4.8 \mathrm{~Hz}, 2 \mathrm{H}), 5.90(\mathrm{dd}, J=5.8,2.5 \mathrm{~Hz}, 1 \mathrm{H}), 5.39-$ $5.27(\mathrm{~m}, 2 \mathrm{H}), 5.26-5.11(\mathrm{~m}, 1 \mathrm{H}), 4.33(\mathrm{dd}, J=12.5,4.3 \mathrm{~Hz}$, $1 \mathrm{H}), 4.14$ (dd, $J=12.5,2.4 \mathrm{~Hz}, 1 \mathrm{H}), 3.94$ (ddd, $J=10.1,4.4$, $2.2 \mathrm{~Hz}, 1 \mathrm{H}), 2.07$ (s, 3H), 2.05 (s, 3H), 2.04 (s, 3H), 1.99 (s, $3 \mathrm{H})$, no $\mathrm{OH}$ proton observed; ${ }^{13} \mathbf{C}\{\mathbf{1 H}\}$ NMR (75 MHz, $\left.\mathbf{C D C l}_{3}\right) \boldsymbol{\delta}(\mathbf{p p m}) 170.9\left(\mathrm{C}_{\mathrm{q}}\right), 170.3\left(\mathrm{C}_{\mathrm{q}}\right), 169.8\left(\mathrm{C}_{\mathrm{q}}\right), 169.7$ $\left(\mathrm{C}_{\mathrm{q}}\right), 164.3\left(\mathrm{C}_{\mathrm{q}}\right), 161.1\left(\mathrm{C}_{\mathrm{q}}\right), 132.8(2 \times \mathrm{CH}), 120.8\left(\mathrm{C}_{\mathrm{q}}\right), 115.6$ 
(2 x CH), $92.3(\mathrm{CH}), 72.9(\mathrm{CH}), 72.8(\mathrm{CH}), 70.4(\mathrm{CH}), 68.1$ $(\mathrm{CH}), 61.7\left(\mathrm{CH}_{2}\right), 20.9\left(\mathrm{CH}_{3}\right), 20.7\left(3 \times \mathrm{CH}_{3}\right)$; HRMS (ESI) $(\mathbf{M}+\mathbf{N a})^{+} \mathrm{m} / \mathrm{z}$ calculated for $\mathrm{C}_{21} \mathrm{H}_{24} \mathrm{O}_{12} \mathrm{Na} 491.1165$, found 491.1174 .

\section{$\beta$-D-Glucopyranose, 2,3,4,6-tetraacetate 1-(2-bromobenzoate) [7i]}

Compound 7i was prepared by using both general procedures (A) (reaction time: $15 \mathrm{~min}$ ), and (B) (reaction time: 1h), with commercial 2-bromobenzoic acid $\mathbf{5 i}(38.6 \mathrm{mg}, 0.19 \mathrm{mmol}, 1.0$ equiv.) and 1a (104.9 mg, $0.29 \mathrm{mmol}, 1.5$ equiv.), which afforded the product as a white solid (with $\mathrm{Cu}$-catalyst 0.091 mmol, $48 \%$ yield, with Co-catalyst $0.057 \mathrm{mmol}, 30 \%$ yield) after column chromatography EtOAc/Cyclohexane 3:7): TLC $\mathbf{R}_{\mathbf{f}}=0.47\left(\right.$ EtOAc/Cyclohexane, $\left.1: 1, \mathrm{SiO}_{2}\right) ; \mathbf{m p}: 103.3-105.2$ ${ }^{\circ} \mathrm{C} ;[\boldsymbol{\alpha}]_{\mathbf{D}}{ }^{\mathbf{2 0}}=-13.6\left(\mathrm{c}, 1.00\right.$ in $\left.\mathrm{CHCl}_{3}\right)$; IR (neat, $\mathbf{c m}^{-\mathbf{1}}$ ) 2959, 1758, 1590, 1433, 1366, 1235, 1070, 1031; ${ }^{1}$ H NMR (300 $\left.\mathbf{M H z}, \mathbf{C D C l}_{3}\right) \boldsymbol{\delta}$ (ppm) $7.93-7.83(\mathrm{~m}, 1 \mathrm{H}), 7.75-7.61(\mathrm{~m}$, $1 \mathrm{H}), 7.38(\mathrm{dd}, J=6.8,3.5 \mathrm{~Hz}, 2 \mathrm{H}), 6.03-5.90(\mathrm{~m}, 1 \mathrm{H}), 5.37$ $-5.26(\mathrm{~m}, 2 \mathrm{H}), 5.26-5.09(\mathrm{~m}, 1 \mathrm{H}), 4.34(\mathrm{dd}, J=12.5,4.4$ $\mathrm{Hz}, 1 \mathrm{H}), 4.14$ (dd, J = 12.6, $2.2 \mathrm{~Hz}, 1 \mathrm{H}$ ), 3.93 (ddd, $J=12.1$, 4.4, $2.1 \mathrm{~Hz}, 1 \mathrm{H}), 2.08$ (s, 3H), 2.05 (s, 3H), 2.03 (s, 3H), 2.02 $(\mathrm{s}, 3 \mathrm{H}) ;{ }^{13} \mathbf{C}\{\mathbf{1 H}\}$ NMR (75 MHz, $\left.\mathbf{C D C l}_{3}\right) \boldsymbol{\delta}(\mathbf{p p m}) 170.8\left(\mathrm{C}_{\mathrm{q}}\right)$, $170.2\left(\mathrm{C}_{\mathrm{q}}\right), 169.5\left(2 \times \mathrm{C}_{\mathrm{q}}\right), 163.4\left(\mathrm{C}_{\mathrm{q}}\right), 135.1(\mathrm{CH}), 133.9$ $(\mathrm{CH}), 132.5(\mathrm{CH}), 129.5\left(\mathrm{C}_{\mathrm{q}}\right), 127.6(\mathrm{CH}), 123.0\left(\mathrm{C}_{\mathrm{q}}\right), 92.6$ $(\mathrm{CH}), 73.0(\mathrm{CH}), 72.9(\mathrm{CH}), 70.3(\mathrm{CH}), 67.9(\mathrm{CH}), 61.6$ $\left(\mathrm{CH}_{2}\right), 20.9\left(\mathrm{CH}_{3}\right), 20.8\left(\mathrm{CH}_{3}\right), 20.7\left(2 \times \mathrm{CH}_{3}\right)$; HRMS (ESI) $(\mathbf{M}+\mathbf{N a})^{+} \mathrm{m} / z$ calculated for $\mathrm{C}_{21} \mathrm{H}_{23} \mathrm{BrO}_{11} \mathrm{Na} 553.0321$, found 553.0327 .

$\beta$-D-Glucopyranose, 2,3,4,6-tetraacetate 1-(2-fluorobenzoate) [7j]

Compound $7 \mathbf{j}$ was prepared by using both general procedures (A) (reaction time: $15 \mathrm{~min}$ ), and (B) (reaction time: $1 \mathrm{~h}$ ), with commercial 2-fluorobenzoic acid 5j (26.9 mg, $0.19 \mathrm{mmol}, 1.0$ equiv.) and 1a (104.9 mg, $0.29 \mathrm{mmol}, 1.5$ equiv.), which afforded the product as a white solid (with $\mathrm{Cu}$-catalyst 0.114 mmol, $60 \%$ yield, with Co-catalyst $0.112 \mathrm{mmol}, 59 \%$ yield) after column chromatography EtOAc/Cyclohexane 3:7): TLC $\mathbf{R}_{\mathbf{f}}=0.46\left(\right.$ EtOAc/Cyclohexane, $\left.1: 1, \mathrm{SiO}_{2}\right) ; \mathbf{m p}: 116.6-118.0$ ${ }^{\circ} \mathrm{C} ;[\boldsymbol{\alpha}]_{\mathbf{D}}{ }^{\mathbf{2 0}}=-14.0\left(\mathrm{c}, 1.00\right.$ in $\mathrm{CHCl}_{3}$ ); IR (neat, $\mathbf{c m}^{-1}$ ) 3496, 2954, 1745, 1613, 1491, 1367, 1240, 1081, $1036 ;{ }^{1} \mathbf{H}$ NMR $\left(300 \mathbf{M H z}_{1} \mathbf{C D C l}_{3}\right) \boldsymbol{\delta}$ (ppm) $7.92(\mathrm{t}, J=7.1 \mathrm{~Hz}, 1 \mathrm{H}), 7.56(\mathrm{dd}$, $J=12.6,7.2 \mathrm{~Hz}, 1 \mathrm{H}), 7.18(\mathrm{dt}, J=19.2,8.6 \mathrm{~Hz}, 2 \mathrm{H}), 5.93(\mathrm{dd}$, $J=6.0,0.9 \mathrm{~Hz}, 1 \mathrm{H}), 5.38-5.26(\mathrm{~m}, 2 \mathrm{H}), 5.26-5.11(\mathrm{~m}, 1 \mathrm{H})$, $4.32(\mathrm{dd}, J=12.5,4.3 \mathrm{~Hz}, 1 \mathrm{H}), 4.14(\mathrm{~d}, J=12.3 \mathrm{~Hz}, 1 \mathrm{H}), 4.03$ $-3.84(\mathrm{~m}, 1 \mathrm{H}), 2.07(\mathrm{~s}, 3 \mathrm{H}), 2.04(\mathrm{~s}, 3 \mathrm{H}), 2.03(\mathrm{~s}, 3 \mathrm{H}), 2.01(\mathrm{~s}$, $3 \mathrm{H}) ;{ }^{13} \mathbf{C}\{1 \mathrm{H}\}$ NMR (75 MHz, $\left.\mathbf{C D C l}_{3}\right) \boldsymbol{\delta}$ (ppm) $170.7\left(\mathrm{C}_{\mathrm{q}}\right)$, $170.2\left(\mathrm{C}_{\mathrm{q}}\right), 169.5\left(\mathrm{C}_{\mathrm{q}}\right), 169.4\left(\mathrm{C}_{\mathrm{q}}\right), 162.6\left(\mathrm{~d}, J_{C F}=261.0 \mathrm{~Hz}\right.$, $\left.\mathrm{C}_{\mathrm{q}}\right), 162.0\left(\mathrm{~d}, J_{C F}=3.8 \mathrm{~Hz}, \mathrm{C}_{\mathrm{q}}\right), 135.7\left(\mathrm{~d}, J_{C F}=9.0 \mathrm{~Hz}, \mathrm{CH}\right)$, $132.5(\mathrm{CH}), 124.3\left(\mathrm{~d}, J_{C F}=3.0 \mathrm{~Hz}, \mathrm{CH}\right), 117.3\left(\mathrm{C}_{\mathrm{q}}\right), 117.3(\mathrm{~d}$, $\left.J_{C F}=21.8 \mathrm{~Hz}, \mathrm{CH}\right), 92.4(\mathrm{CH}), 73.0(\mathrm{CH}), 72.8(\mathrm{CH}), 70.2$ $(\mathrm{CH}), 68.0(\mathrm{CH}), 61.6\left(\mathrm{CH}_{2}\right), 20.8\left(\mathrm{CH}_{3}\right), 20.72\left(3 \mathrm{x} \mathrm{CH}_{3}\right) ;{ }^{19} \mathbf{F}$ NMR (376 MHz, $\mathbf{C D C l}_{3}$ ) $\delta$ (ppm) - 108.3 (CF); HRMS (ESI) $(\mathbf{M}+\mathbf{N a})^{+} \mathrm{m} / z$ calculated for $\mathrm{C}_{21} \mathrm{H}_{23} \mathrm{FO}_{11} \mathrm{Na} 493.1122$, found 493.1126 .

\section{$\beta$-D-Glucopyranose, 2,3,4,6-tetraacetate 1-(4-fluorobenzoate)} $[7 \mathrm{k}]$

Compound 7k was prepared by using the general procedure (B) (reaction time: 1h), with commercial 4-fluorobenzoic acid 5k $(26.9 \mathrm{mg}, 0.19 \mathrm{mmol}, 1.0$ equiv.) and $\mathbf{1 a}(104.9 \mathrm{mg}, 0.29$ mmol, 1.5 equiv.), which afforded the product as a white solid
(55.5 mg, 62\% yield after column chromatography EtOAc/Cyclohexane 3:7); TLC $\quad \mathbf{R}_{\mathbf{f}}=0.57$ (EtOAc/Cyclohexane, 1:1, $\mathrm{SiO}_{2}$ ); mp: $157.3-159.7{ }^{\circ} \mathrm{C}$; $[\boldsymbol{\alpha}]_{\mathbf{D}}{ }^{\mathbf{2 0}}=-11.0\left(\mathrm{c}, 1.00\right.$ in $\left.\mathrm{CHCl}_{3}\right)$; IR (neat, $\left.\mathbf{c m}^{-\mathbf{1}}\right)$ 2964, 2924, 1759, 1604, 1509, 1366, 1242, 1067, 1034; ${ }^{1} \mathbf{H}$ NMR (300 MHz, $\left.\mathbf{C D C l}_{3}\right) \boldsymbol{\delta}(\mathbf{p p m}) 8.05(\mathrm{dd}, J=8.8,5.4 \mathrm{~Hz}, 2 \mathrm{H}), 7.13(\mathrm{t}$, $J=8.6 \mathrm{~Hz}, 2 \mathrm{H}), 5.90(\mathrm{dd}, J=4.9,3.3 \mathrm{~Hz}, 1 \mathrm{H}), 5.33(\mathrm{dd}, J=$ $5.6,3.1 \mathrm{~Hz}, 2 \mathrm{H}), 5.27-5.09(\mathrm{~m}, 1 \mathrm{H}), 4.32(\mathrm{dd}, J=12.5,4.4$ $\mathrm{Hz}, 1 \mathrm{H}), 4.13$ (dd, $J=12.6,2.4 \mathrm{~Hz}, 1 \mathrm{H}$ ), 3.93 (ddd, $J=9.9$, 4.5, $2.2 \mathrm{~Hz}, 1 \mathrm{H}), 2.07$ (s, 3H), 2.04 (s, 3H), 2.04 (s, 3H), 1.98 $(\mathrm{s}, 3 \mathrm{H}) ;{ }^{13} \mathbf{C}\{\mathbf{1 H}\}$ NMR (75 MHz, $\left.\mathbf{C D C l}_{3}\right) \boldsymbol{\delta}(\mathbf{p p m}) 170.7\left(\mathrm{C}_{\mathrm{q}}\right)$, $170.2\left(\mathrm{C}_{\mathrm{q}}\right), 169.6\left(\mathrm{C}_{\mathrm{q}}\right), 169.5\left(\mathrm{C}_{\mathrm{q}}\right), 166.5\left(\mathrm{~d}, J_{C F}=254.3 \mathrm{~Hz}\right.$, $\left.\mathrm{C}_{\mathrm{q}}\right), 163.7\left(\mathrm{C}_{\mathrm{q}}\right), 133.0\left(\mathrm{~d}, J_{C F}=9.0 \mathrm{~Hz}, 2 \times \mathrm{CH}\right), 124.8\left(\mathrm{~d}, J_{C F}\right.$ $\left.=3.0 \mathrm{~Hz}, \mathrm{C}_{\mathrm{q}}\right), 116.1\left(\mathrm{~d}, J_{C F}=21.8 \mathrm{~Hz}, 2 \times \mathrm{CH}\right), 92.5(\mathrm{CH})$, $72.9(\mathrm{CH}), 72.7(\mathrm{CH}), 70.3(\mathrm{CH}), 68.0(\mathrm{CH}), 61.6\left(\mathrm{CH}_{2}\right), 20.8$ $\left(\mathrm{CH}_{3}\right), 20.7\left(3 \times \mathrm{CH}_{3}\right) ;{ }^{19} \mathbf{F}$ NMR (376 MHz, $\left.\mathbf{C D C l}_{3}\right) \delta$ (ppm) - 103.4 (CF); HRMS (ESI) $(\mathbf{M}+\mathbf{N a})^{+} \mathrm{m} / z$ calculated for $\mathrm{C}_{21} \mathrm{H}_{23} \mathrm{FO}_{11} \mathrm{Na}$ 493.1122, found 493.1120.

$\beta$-D-Glucopyranose, 2,3,4,6-tetraacetate 1-(4-iodobenzoate) [71]

Compound 7l was prepared by using both general procedures (A) (reaction time: $15 \mathrm{~min}$ ), and (B) (reaction time: $1 \mathrm{~h}$ ), with commercial 4-iodobenzoic acid $\mathbf{5 l}(47.6 \mathrm{mg}, 0.19 \mathrm{mmol}, 1.0$ equiv.) and 1a (104.9 mg, $0.29 \mathrm{mmol}, 1.5$ equiv.), which afforded the product as a white solid (with $\mathrm{Cu}$-catalyst 0.123 mmol, $65 \%$ yield, with Co-catalyst $0.081 \mathrm{mmol}, 43 \%$ yield) after column chromatography EtOAc/Cyclohexane 3:7): TLC $\mathbf{R}_{\mathbf{f}}=0.59\left(\right.$ EtOAc/Cyclohexane, $\left.1: 1, \mathrm{SiO}_{2}\right) ; \mathbf{m p :} 149.0-151.6$ ${ }^{\circ} \mathrm{C} ;[\boldsymbol{\alpha}]_{\mathbf{D}}{ }^{19}=-21.9\left(\mathrm{c}, 1.00\right.$ in $\left.\mathrm{CHCl}_{3}\right)$; IR (neat, $\mathbf{c m}^{-\mathbf{1}}$ ) 2949, 1746, 1586, 1366, 1229, 1085, 1069; ${ }^{\mathbf{1}} \mathbf{H}$ NMR (300 MHz, $\left.\mathbf{C D C l}_{3}\right) \boldsymbol{\delta}$ (ppm) $7.83(\mathrm{dd}, J=8.5,2.1 \mathrm{~Hz}, 2 \mathrm{H}), 7.73(\mathrm{dd}, J=$ $8.5,2.0 \mathrm{~Hz}, 2 \mathrm{H}), 5.89$ (d, $J=6.8 \mathrm{~Hz}, 1 \mathrm{H}), 5.38-5.26(\mathrm{~m}, 2 \mathrm{H})$, $5.26-5.11(\mathrm{~m}, 1 \mathrm{H}), 4.32(\mathrm{dd}, J=12.6,4.5 \mathrm{~Hz}, 1 \mathrm{H}), 4.13(\mathrm{dd}$, $J=12.5,2.1 \mathrm{~Hz}, 1 \mathrm{H}), 3.93(\mathrm{ddd}, J=10.0,4.6,2.3 \mathrm{~Hz}, 1 \mathrm{H})$, 2.07 (s, 3H), 2.05 (s, 3H), 2.04 (s, 3H), 1.98 (s, 3H); ${ }^{13} \mathbf{C}\{\mathbf{1 H}\}$ NMR (75 MHz, $\left.\mathbf{C D C l}_{3}\right) \boldsymbol{\delta}$ (ppm) $170.7\left(\mathrm{C}_{\mathrm{q}}\right), 170.2\left(\mathrm{C}_{\mathrm{q}}\right)$, $169.6\left(\mathrm{C}_{\mathrm{q}}\right), 169.5\left(\mathrm{C}_{\mathrm{q}}\right), 164.3\left(\mathrm{C}_{\mathrm{q}}\right), 138.2(2 \times \mathrm{CH}), 131.6(2 \mathrm{x}$ $\mathrm{CH}), 128.0\left(\mathrm{C}_{\mathrm{q}}\right), 102.4\left(\mathrm{C}_{\mathrm{q}}\right), 92.6(\mathrm{CH}), 73.0(\mathrm{CH}), 72.7(\mathrm{CH})$, $70.2(\mathrm{CH}), 68.0(\mathrm{CH}), 61.5\left(\mathrm{CH}_{2}\right), 20.8\left(\mathrm{CH}_{3}\right), 20.7\left(3 \times \mathrm{CH}_{3}\right)$; HRMS (ESI) $(\mathbf{M}+\mathbf{N a})^{+} \mathrm{m} / z$ calculated for $\mathrm{C}_{21} \mathrm{H}_{23} \mathrm{IO}_{11} \mathrm{Na}$ 601.0183 , found 601.0183 .

\section{$\beta$-D-Glucopyranose, $\quad$ 2,3,4,6-tetraacetate $\quad 1-(3,5-$ dichlorobenzoate) [7m]}

Compound 7m was prepared by using both general procedures (A) (reaction time: $15 \mathrm{~min}$ ), and (B) (reaction time: $1 \mathrm{~h}$ ), with commercial 3,5-dichlorobenzoic acid 5m (36.7 mg, 0.19 mmol, 1.0 equiv.) and 1a (104.9 mg, $0.29 \mathrm{mmol}, 1.5$ equiv.), which afforded the product as a white solid (with $\mathrm{Cu}$-catalyst $0.104 \mathrm{mmol}, 55 \%$ yield, with Co-catalyst $0.157 \mathrm{mmol}, 83 \%$ yield) after column chromatography EtOAc/Cyclohexane 3:7): TLC $\mathbf{R}_{\mathbf{f}}=0.59\left(\right.$ EtOAc/Cyclohexane, $\left.1: 1, \mathrm{SiO}_{2}\right)$; mp: $87.8-$ $89.5{ }^{\circ} \mathrm{C} ;[\boldsymbol{\alpha}]_{\mathbf{D}}{ }^{20}=-12.7$ (c, 1.00 in $\mathrm{CHCl}_{3}$ ); IR (neat, $\mathbf{~ c m}^{-\mathbf{1}}$ ) 2964, 2920, 1747, 1571, 1426, 1366, 1207, 1073, 1030; ${ }^{1} \mathbf{H}$ NMR (300 MHz, CDCl M $^{\delta}$ (ppm) 7.89 (d, $\left.J=2.1 \mathrm{~Hz}, 2 \mathrm{H}\right)$, $7.57(\mathrm{t}, J=2.1 \mathrm{~Hz}, 1 \mathrm{H}), 5.91(\mathrm{dd}, J=5.0,2.7 \mathrm{~Hz}, 1 \mathrm{H}), 5.39-$ $5.25(\mathrm{~m}, 2 \mathrm{H}), 5.17(\mathrm{ddd}, J=9.3,6.0,3.1 \mathrm{~Hz}, 1 \mathrm{H}), 4.30(\mathrm{dd}, J$ $=12.5,4.5 \mathrm{~Hz}, 1 \mathrm{H}), 4.12(\mathrm{dd}, J=12.6,2.3 \mathrm{~Hz}, 1 \mathrm{H}), 3.92(\mathrm{ddd}$, $J=10.1,4.6,2.3 \mathrm{~Hz}, 1 \mathrm{H}), 2.06(\mathrm{~s}, 3 \mathrm{H}), 2.04(\mathrm{~s}, 3 \mathrm{H}), 2.03(\mathrm{~s}$, $3 \mathrm{H}), 1.99$ (s, 3H); ${ }^{\mathbf{1 3}} \mathbf{C}\{\mathbf{1 H}\}$ NMR (75 MHz, $\left.\mathbf{C D C l}_{3}\right) \boldsymbol{\delta}$ (ppm) $170.7\left(\mathrm{C}_{\mathrm{q}}\right), 170.2\left(\mathrm{C}_{\mathrm{q}}\right), 169.5\left(\mathrm{C}_{\mathrm{q}}\right), 169.3\left(\mathrm{C}_{\mathrm{q}}\right), 162.5\left(\mathrm{C}_{\mathrm{q}}\right)$, $135.7\left(2 \times \mathrm{C}_{\mathrm{q}}\right), 133.9(\mathrm{CH}), 131.4\left(\mathrm{C}_{\mathrm{q}}\right), 128.6(2 \times \mathrm{CH}), 92.9$ 
$(\mathrm{CH}), 73.0(\mathrm{CH}), 72.6(\mathrm{CH}), 70.3(\mathrm{CH}), 67.9(\mathrm{CH}), 61.5$ $\left(\mathrm{CH}_{2}\right), 20.8\left(\mathrm{CH}_{3}\right), 20.7\left(3 \mathrm{x} \mathrm{CH}_{3}\right) ;$ HRMS (ESI) $(\mathbf{M}+\mathbf{N a})^{+}$ $m / z$ calculated for $\mathrm{C}_{21} \mathrm{H}_{22} \mathrm{Cl}_{2} \mathrm{O}_{11} \mathrm{Na}$ 543.0437, found 543.0432.

$\beta$-D-Glucopyranose, $\quad$ 2,3,4,6-tetraacetate $\quad 1-(2,4$ dichlorobenzoate) [7n]

Compound $\mathbf{7 n}$ was prepared by using the general procedure (A) (reaction time: 15min), with commercial 2,3-dichlorobenzoic acid $\mathbf{5 n}$ (36.7 $\mathrm{mg}, 0.19 \mathrm{mmol}, 1.0$ equiv.) and $1 \mathrm{a}$ (104.9 $\mathrm{mg}, 0.29 \mathrm{mmol}, 1.5$ equiv.), which afforded the product as a amorphos solid $(0.097 \mathrm{mmol}, 55 \%$ yield after column chromatography EtOAc/Cyclohexane 4:6); $\mathbf{R}_{\mathbf{f}}(40 \%$ EtOAc/Cyclohexane $)=0.50 ;[\boldsymbol{\alpha}]_{\mathbf{D}}{ }^{25}=-37.27$ (c 0,011 $\mathrm{CH}_{2} \mathrm{Cl}_{2}$ ); IR (neat, $\mathbf{c m}^{-\mathbf{1}}$ ) 3460, 1745, 1711, 1584, 1376, 1207, 1071, 1021; ${ }^{\mathbf{H}} \mathbf{H}$ NMR (300 MHz, $\left.\mathbf{C D C l}_{3}\right) \delta$ (ppm) $7.85(\mathrm{~d}, J$ $=8.5 \mathrm{~Hz}, 1 \mathrm{H}), 7.49-7.45(\mathrm{~m}, J=1.4 \mathrm{~Hz}, 1 \mathrm{H}), 7.31(\mathrm{~d}, J=8.5$ $\mathrm{Hz}, 1 \mathrm{H}), 5.92(\mathrm{~d}, J=7.5 \mathrm{~Hz}, 1 \mathrm{H}), 5.29(\mathrm{t}, J=6.6 \mathrm{~Hz}, 2 \mathrm{H})$, $5.16(\mathrm{t}, J=9.3 \mathrm{~Hz}, 1 \mathrm{H}), 4.31(\mathrm{dd}, J=12.5,4.3 \mathrm{~Hz}, 1 \mathrm{H}), 4.15-$ $4.07(\mathrm{~m}, 1 \mathrm{H}), 3.95-3.88(\mathrm{~m}, 1 \mathrm{H}), 2.05(\mathrm{~s}, 3 \mathrm{H}), 2.02(\mathrm{~s}, 3 \mathrm{H})$, $2.01(\mathrm{~s}, 3 \mathrm{H}), 1.99(\mathrm{~s}, 3 \mathrm{H}) ;{ }^{13} \mathbf{C}\{\mathbf{1 H}\} \mathbf{N M R}\left(\mathbf{7 5} \mathbf{~ M H z}, \mathbf{C D C l}_{\mathbf{3}}\right) \delta$ (ppm) $170.52\left(\mathrm{C}_{\mathrm{q}}\right), \quad 170.00\left(\mathrm{C}_{\mathrm{q}}\right), \quad 169.35\left(\mathrm{C}_{\mathrm{q}}\right), \quad 169.29\left(\mathrm{C}_{\mathrm{q}}\right)$, $161.96\left(\mathrm{C}_{\mathrm{q}}\right), 139.68\left(\mathrm{C}_{\mathrm{q}}\right), 136.07\left(\mathrm{C}_{\mathrm{q}}\right), 133.26(\mathrm{CH}), 131.41(\mathrm{CH})$, 127.30(CH), 125.77( $\left(\mathrm{C}_{\mathrm{q}}\right), 92.45(\mathrm{CH}), 72.86(\mathrm{CH}), 72.63(\mathrm{CH})$, 70.12(CH), 67.73(CH), 61.37 $\left(\mathrm{CH}_{2}\right), 20.66\left(\mathrm{CH}_{3}\right), 20.54(3 \mathrm{x}$ $\left.\mathrm{CH}_{3}\right)$; HRMS (ESI) $(\mathbf{M}+\mathbf{N a})^{+} \mathrm{m} / \mathrm{z}$ calculated for $\mathrm{C}_{21} \mathrm{H}_{22} \mathrm{O}_{11} \mathrm{NaCl}_{2} \mathrm{Na}$ : 543.0437, found 543.0432.

\section{$\beta$-D-Glucopyranose, 2,3,4,6-tetraacetate $\quad 1-(2,5$ - difluorobenzoate) [7o]}

Compound 7o was prepared by using the general procedure $\mathbf{A}$ (reaction time: 15min), with commercial 2,5-difluorobenzoic acid 50 (36.7 mg, $0.19 \mathrm{mmol}, 1.0$ equiv.) and 1a (104.9 mg, $0.29 \mathrm{mmol}, 1.5$ equiv.), which afforded the product as a white solid $(0.118$ mmol, $62 \%$ yield after column chromatography EtOAc/Cyclohexane 4:6); $\mathbf{R}_{\mathbf{f}}(40 \%$ EtOAc/Cyclohexane $)=$ 0.39 ; mp: $105-112{ }^{\circ} \mathrm{C} ;[\boldsymbol{\alpha}]_{\mathbf{D}}{ }^{25}=-37.73\left(\mathrm{c} 0,0053\right.$ in $\left.\mathrm{CH}_{2} \mathrm{Cl}_{2}\right)$; IR (neat, $\mathbf{c m}^{-1}$ ) 1758, 1739, 1496, 1381, 1209, 1185, 1077, 1035; ${ }^{1} \mathbf{H}$ NMR (300 $\left.\mathbf{M H z}, \mathbf{C D C l}_{3}\right) \delta(\mathbf{p p m}) 7.64-7.55(\mathrm{~m}$, $1 \mathrm{H}), 7.30-7.21(\mathrm{~m}, 1 \mathrm{H}), 7.16-7.10(\mathrm{~m}, J=9.3,4.1 \mathrm{~Hz}, 1 \mathrm{H})$, $5.91(\mathrm{~d}, J=7.4 \mathrm{~Hz}, 1 \mathrm{H}), 5.33-5.27(\mathrm{~m}, J=3.4 \mathrm{~Hz}, 2 \mathrm{H}), 5.21$ $-5.12(\mathrm{~m}, J=9.5 \mathrm{~Hz}, 1 \mathrm{H}), 4.30(\mathrm{dd}, J=12.5,4.4 \mathrm{~Hz}, 1 \mathrm{H})$, $4.13(\mathrm{~d}, J=12.1 \mathrm{~Hz}, 1 \mathrm{H}), 3.96-3.89(\mathrm{~m}, J=9.8 \mathrm{~Hz}, 1 \mathrm{H})$, $2.06(\mathrm{~s}, 3 \mathrm{H}), 2.03-2.00(\mathrm{~m}, 9 \mathrm{H}) ;{ }^{13} \mathbf{C}\{\mathbf{1 H}\}$ NMR (75 MHz,

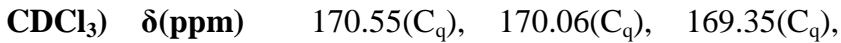
$169.15\left(\mathrm{C}_{\mathrm{q}}\right), 161.00\left(\mathrm{C}_{\mathrm{q}}\right), 160.15\left(\mathrm{C}_{\mathrm{q}}\right), 159.59\left(\mathrm{C}_{\mathrm{q}}\right), 156.31\left(\mathrm{C}_{\mathrm{q}}\right)$, 122.29(CH), $118.55(\mathrm{CH}), \quad 118.21(\mathrm{CH}), \quad 92.50(\mathrm{CH})$, $72.85(\mathrm{CH}), 72.59(\mathrm{CH}), 70.00(\mathrm{CH}), \quad 67.75(\mathrm{CH}), 61.40\left(\mathrm{CH}_{2}\right)$, 20.65 $\left(\mathrm{CH}_{3}\right), 20.55\left(2 \times \mathrm{CH}_{3}\right), 20.48\left(\mathrm{CH}_{3}\right) ;{ }^{19} \mathbf{F}$ NMR (376 $\left.\mathbf{M H z}, \mathbf{C D C l}_{3}\right) \boldsymbol{\delta}$ (ppm) -113.91(CF), -117.23 (CF) ; HRMS (ESI) $(\mathbf{M}+\mathbf{N a})^{+} \mathrm{m} / z$ calculated for $\mathrm{C}_{21} \mathrm{H}_{22} \mathrm{O}_{11} \mathrm{~F}_{2} \mathrm{Na}$ : 511.1022, found 511.1028 .

\section{$\beta$-D-Glucopyranose, 2,3,4,6-tetraacetate 1-(2-nitrobenzoate)} [7p]

Compound $\mathbf{7 p}$ was prepared by using the general procedure (B) (reaction time: 1h), with commercial 2-nitrobenzoic acid $\mathbf{5 p}$ (32.1 mg, 0.19 mmol, 1.0 equiv.) and $\mathbf{1 a}(104.9 \mathrm{mg}, 0.29$ mmol, 1.5 equiv.), which afforded the product as a white solid (55.0 mg, 58\% yield after column chromatography EtOAc/Cyclohexane 3:7); $\quad$ TLC $\quad \mathbf{R}_{\mathbf{f}}=0.36$ (EtOAc/Cyclohexane, $\left.1: 1, \mathrm{SiO}_{2}\right)$; $\mathbf{~ m p : ~} 74.9-75.6{ }^{\circ} \mathrm{C} ;[\boldsymbol{\alpha}]_{\mathbf{D}}{ }^{20}$ $=-8.5\left(\mathrm{c}, 1.00\right.$ in $\left.\mathrm{CHCl}_{3}\right)$; IR (neat, $\mathbf{~ c m}^{-1}$ ) 2954, 2929, 1757,
1536, 1367, 1351, 1242, 1063, 1035; ${ }^{\mathbf{1}} \mathbf{H}$ NMR (300 MHz, $\left.\mathbf{C D C l}_{3}\right) \boldsymbol{\delta}$ (ppm) $7.96(\mathrm{~d}, J=7.4 \mathrm{~Hz}, 1 \mathrm{H}), 7.78-7.61(\mathrm{~m}, 3 \mathrm{H})$, $5.93(\mathrm{~d}, J=8.2 \mathrm{~Hz}, 1 \mathrm{H}), 5.32(\mathrm{t}, J=9.4 \mathrm{~Hz}, 1 \mathrm{H}), 5.16(\mathrm{dd}, J=$ 20.6, $9.1 \mathrm{~Hz}, 2 \mathrm{H}), 4.33$ (dd, $J=12.5,4.4 \mathrm{~Hz}, 1 \mathrm{H}), 4.15$ (dd, $J$ $=12.0,1.8 \mathrm{~Hz}, 1 \mathrm{H}), 4.02-3.82(\mathrm{~m}, 1 \mathrm{H}), 2.11(\mathrm{~s}, 3 \mathrm{H}), 2.08(\mathrm{~s}$, 3H), $2.04(\mathrm{~s}, 3 \mathrm{H}), 2.01(\mathrm{~s}, 3 \mathrm{H}) ;{ }^{13} \mathbf{C}\{\mathbf{1 H}\}$ NMR (75 MHz, $\left.\mathbf{C D C l}_{3}\right) \boldsymbol{\delta}(\mathbf{p p m}) 170.7\left(\mathrm{C}_{\mathrm{q}}\right), 170.2\left(\mathrm{C}_{\mathrm{q}}\right), 169.7\left(\mathrm{C}_{\mathrm{q}}\right), 169.5$ $\left(\mathrm{C}_{\mathrm{q}}\right), 163.9\left(\mathrm{C}_{\mathrm{q}}\right), 147.8\left(\mathrm{C}_{\mathrm{q}}\right), 133.4(\mathrm{CH}), 132.5(\mathrm{CH}), 130.2$ $(\mathrm{CH}), 126.5\left(\mathrm{C}_{\mathrm{q}}\right), 124.3(\mathrm{CH}), 93.1(\mathrm{CH}), 73.0(\mathrm{CH}), 72.8$ $(\mathrm{CH}), 69.9(\mathrm{CH}), 67.8(\mathrm{CH}), 61.5\left(\mathrm{CH}_{2}\right), 20.8\left(2 \times \mathrm{CH}_{3}\right), 20.7$ $\left(2 \mathrm{x} \mathrm{CH}_{3}\right) ; \mathbf{H R M S}$ (ESI) $(\mathbf{M}+\mathbf{N a})^{+} \mathrm{m} / \mathrm{z}$ calculated for $\mathrm{C}_{21} \mathrm{H}_{23} \mathrm{NO}_{13} \mathrm{Na}$ 520.1067, found 520.1066.

$\beta$-D-Glucopyranose, 2,3,4,6-tetraacetate 1 -(2-amino, 3,5dibromo, 6-chlorobenzoate) [7q]

Compound $\mathbf{7 q}$ was prepared by using the general procedure (A) (reaction time: $15 \mathrm{~min}$ ), with commercial 2-amino-3,5dibromo-6-chlorobenzoic acid $\mathbf{5 q}(63.24 \mathrm{mg}, 0.19 \mathrm{mmol}, 1.0$ equiv.) and $1 \mathrm{a}$ (104.9 $\mathrm{mg}, 0.29 \mathrm{mmol}, 1.5$ equiv.), which afforded the product as a white solid $(0.09 \mathrm{mmol}, 47 \%$ yield after column chromatography EtOAc/Cyclohexane 4:6); $\mathbf{R}_{\mathbf{f}}$ $(40 \%$ EtOAc/Cyclohexane $)=0.38 ; \mathbf{~ m p : ~} 213-219{ }^{\circ} \mathrm{C} ;[\boldsymbol{\alpha}]_{\mathbf{D}}{ }^{25}=$ -19.6 (c 0,0051 in $\mathrm{CH}_{2} \mathrm{Cl}_{2}$ ); IR (neat, $\mathbf{c m}^{-1}$ ) 3371, 2925, 1756, 1730, 1442, 1368, 1245, 1111, 1083, 1032; ${ }^{\mathbf{1}} \mathbf{H}$ NMR (300 MHz, $\left.\mathbf{C D C l}_{3}\right) \delta(\mathbf{p p m}) 7.73(\mathrm{~s}, 1 \mathrm{H}), 6.00(\mathrm{~d}, J=7.9 \mathrm{~Hz}, 1 \mathrm{H})$, $5.27(\mathrm{t}, 1 \mathrm{H}), 5.21(\mathrm{~d}, J=9.3 \mathrm{~Hz}, 1 \mathrm{H}), 5.15(\mathrm{~d}, J=5.8 \mathrm{~Hz}, 2 \mathrm{H})$, $4.30(\mathrm{dd}, J=12.5,4.6 \mathrm{~Hz}, 1 \mathrm{H}), 4.19-4.08(\mathrm{~m}, 2 \mathrm{H}), 3.95-$ $3.87(\mathrm{~m}, 1 \mathrm{H}), 2.06(\mathrm{~s}, 3 \mathrm{H}), 2.03(\mathrm{~s}, 6 \mathrm{H}), 2.00(\mathrm{~s}, 3 \mathrm{H}) ;{ }^{13} \mathbf{C}\{\mathbf{1 H}\}$ NMR (75 MHz, $\left.\mathbf{C D C l}_{3}\right) \delta(\mathbf{p p m}) 170.53\left(\mathrm{C}_{\mathrm{q}}\right), 170.06\left(\mathrm{C}_{\mathrm{q}}\right)$, 169.27( $\left(\mathrm{C}_{\mathrm{q}}\right), 169.16\left(\mathrm{C}_{\mathrm{q}}\right), 163.88\left(\mathrm{C}_{\mathrm{q}}\right), 144.48\left(\mathrm{C}_{\mathrm{q}}\right), 138.34(\mathrm{CH})$, 132.37 $\left(\mathrm{C}_{\mathrm{q}}\right), 115.83\left(\mathrm{C}_{\mathrm{q}}\right), 110.31\left(\mathrm{C}_{\mathrm{q}}\right), 109.00\left(\mathrm{C}_{\mathrm{q}}\right), 92.71(\mathrm{CH})$, 73.05(CH), 72.75(CH), 69.99(CH), 67.69(CH), 61.32( $\left.\mathrm{CH}_{2}\right)$, 20.67 $\left(\mathrm{CH}_{3}\right), 20.54\left(3 \times \mathrm{CH}_{3}\right)$; HRMS (ESI) $(\mathbf{M}+\mathbf{N a})^{+} \mathrm{m} / \mathrm{z}$ calculated for $\mathrm{C}_{21} \mathrm{H}_{22} \mathrm{NO}_{11} \mathrm{ClBr}_{2} \mathrm{Na}$ : 679.9140, found 679.9109.

$\beta$-D-Glucopyranose, 2,3,4,6-tetraacetate 1-(3,5-diiodo, 4 aminobenzoate) [7r]

Compound $\mathbf{7 r}$ was prepared by using the general procedure (B) (reaction time: 1h), with commercial 4-amino-3,5diiodobenzoic acid $\mathbf{5 r}(74.7 \mathrm{mg}, 0.19 \mathrm{mmol}, 1.0$ equiv. $)$ and $\mathbf{1 a}$ (104.9 mg, $0.29 \mathrm{mmol}, 1.5$ equiv.), which afforded the product as a white solid $(64.4 \mathrm{mg}, 47 \%$ yield after column chromatography EtOAc/Cyclohexane 3:7); TLC $\quad \mathbf{R}_{\mathbf{f}}=0.52$ (EtOAc/Cyclohexane, 1:1, $\left.\mathrm{SiO}_{2}\right)$; $\mathbf{m p :} 151.8-152.5{ }^{\circ} \mathrm{C}$; $[\boldsymbol{\alpha}]_{\mathbf{D}}{ }^{\mathbf{2 0}}=-3.0$ (c, 1.00 in $\mathrm{CHCl}_{3}$ ); IR (neat, $\mathbf{c m}^{-1}$ ) 3467, 3359, 2959, 1757, 1610, 1360, 1201, 1060, 1034; ${ }^{\mathbf{1}} \mathbf{H}$ NMR (300 $\left.\mathbf{M H z}, \mathbf{C D C l}_{3}\right) \boldsymbol{\delta}$ (ppm) $8.28(\mathrm{~s}, 2 \mathrm{H}), 5.89(\mathrm{~d}, J=7.4 \mathrm{~Hz}, 1 \mathrm{H})$, $5.34-5.26(\mathrm{~m}, 1 \mathrm{H}), 5.22-5.10(\mathrm{~m}, 2 \mathrm{H}), 4.31(\mathrm{dd}, J=12.5$, $4.3 \mathrm{~Hz}, 1 \mathrm{H}$ ), 4.12 (ddd, $J=12.5,1.2,0.6 \mathrm{~Hz}, 1 \mathrm{H}), 3.91$ (ddd, $J$ $=6.3,3.8,1.5 \mathrm{~Hz}, 1 \mathrm{H}), 2.08(\mathrm{~s}, 3 \mathrm{H}), 2.05(\mathrm{~s}, 3 \mathrm{H}), 2.04(\mathrm{~s}, 3 \mathrm{H})$, $1.99(\mathrm{~s}, 3 \mathrm{H})$, no $\mathrm{NH}_{2}$ protons observed; ${ }^{13} \mathbf{C}\{\mathbf{1 H}\}$ NMR (75 MHz, $\left.\mathbf{C D C l}_{3}\right) \boldsymbol{\delta}$ (ppm) $170.7\left(\mathrm{C}_{\mathrm{q}}\right), 170.2\left(\mathrm{C}_{\mathrm{q}}\right), 169.5\left(\mathrm{C}_{\mathrm{q}}\right)$, $169.4\left(\mathrm{C}_{\mathrm{q}}\right), 169.1\left(\mathrm{C}_{\mathrm{q}}\right), 161.9\left(\mathrm{C}_{\mathrm{q}}\right), 150.7\left(2 \times \mathrm{C}_{\mathrm{q}}\right), 141.7(2 \mathrm{x}$ $\mathrm{CH}), 120.4\left(\mathrm{C}_{\mathrm{q}}\right), 92.3(\mathrm{CH}), 72.8(\mathrm{CH}), 72.7(\mathrm{CH}), 70.3(\mathrm{CH})$, $67.9(\mathrm{CH}), 61.6\left(\mathrm{CH}_{2}\right), 20.8\left(\mathrm{CH}_{3}\right), 20.7\left(3 \times \mathrm{CH}_{3}\right)$; HRMS (ESI) $(\mathbf{M}+\mathbf{N a})^{+} \mathrm{m} / z$ calculated for $\mathrm{C}_{21} \mathrm{H}_{23} \mathrm{I}_{2} \mathrm{NO}_{11} \mathrm{Na} 741.9258$, found 741.9264 .

$\beta$-D-Glucopyranose, 2,3,4,6-tetraacetate 1-acrylate [7s] Compound $\mathbf{7 s}$ was prepared by using the general procedure (B) (reaction time: $1 \mathrm{~h}$ ), with commercial acrylic acid $\mathbf{5 s}(13.8$ 
$\mathrm{mg}, 0.19 \mathrm{mmol}, 1.0$ equiv.) and $1 \mathbf{a}(104.9 \mathrm{mg}, 0.29 \mathrm{mmol}, 1.5$ equiv.), which afforded the product as a white solid $(60.2 \mathrm{mg}$, $78 \%$ yield after column chromatography EtOAc/Cyclohexane 3:7); $\mathbf{T L C} \mathbf{R}_{\mathbf{f}}=0.56\left(\right.$ EtOAc/Cyclohexane, $\left.1: 1, \mathrm{SiO}_{2}\right)$; mp: $107.9-109.4{ }^{\circ} \mathrm{C} ;[\boldsymbol{\alpha}]_{\mathbf{D}}{ }^{\mathbf{2 0}}=-2.7\left(\mathrm{c}, 1.00\right.$ in $\left.\mathrm{CHCl}_{3}\right)$; IR (neat, $\left.\mathbf{c m}^{-1}\right)$ 3457, 2949, 1746, 1368, 1229, 1219, 1079, $1037 ;{ }^{1} \mathbf{H}$ NMR (300 MHz, CDCl $)_{3} \delta$ (ppm) $6.50(\mathrm{~d}, J=17.0 \mathrm{~Hz}, 1 \mathrm{H})$, $6.11(\mathrm{dd}, J=17.1,10.5 \mathrm{~Hz}, 1 \mathrm{H}), 5.96(\mathrm{~d}, J=10.5 \mathrm{~Hz}, 1 \mathrm{H})$, $5.78(\mathrm{~d}, J=8.0 \mathrm{~Hz}, 1 \mathrm{H}), 5.36-5.03(\mathrm{~m}, 3 \mathrm{H}), 4.30(\mathrm{dd}, J=$ $12.5,4.5 \mathrm{~Hz}, 1 \mathrm{H}), 4.11$ (dd, $J=12.4,2.3 \mathrm{~Hz}, 1 \mathrm{H}), 3.87$ (ddd, $J$ $=10.1,4.6,2.3 \mathrm{~Hz}, 1 \mathrm{H}), 2.08(\mathrm{~s}, 3 \mathrm{H}), 2.03(\mathrm{~s}, 3 \mathrm{H}), 2.02(\mathrm{~s}$, 3H), 2.01 (s, 3H); ${ }^{13} \mathbf{C}\{\mathbf{1 H}\}$ NMR (75 MHz, $\mathbf{C D C l}_{3}$ ) $\delta$ (ppm) $170.7\left(\mathrm{C}_{\mathrm{q}}\right), 170.2\left(\mathrm{C}_{\mathrm{q}}\right), 169.5\left(\mathrm{C}_{\mathrm{q}}\right), 169.4\left(\mathrm{C}_{\mathrm{q}}\right), 163.9\left(\mathrm{C}_{\mathrm{q}}\right)$, $133.7\left(\mathrm{CH}_{2}\right), 127.2(\mathrm{CH}), 92.1(\mathrm{CH}), 72.9(\mathrm{CH}), 72.8(\mathrm{CH})$, $70.3(\mathrm{CH}), 68.0(\mathrm{CH}), 61.6\left(\mathrm{CH}_{2}\right), 20.8\left(\mathrm{CH}_{3}\right), 20.7\left(3 \times \mathrm{CH}_{3}\right)$; HRMS (ESI) $(\mathbf{M}+\mathbf{N a})^{+} \mathrm{m} / z$, calculated for $\mathrm{C}_{17} \mathrm{H}_{22} \mathrm{O}_{11} \mathrm{Na}$ 425.1060, found 425.1058 .

\section{$\beta$-D-Glucopyranose, $\quad$ 2,3,4,6-tetraacetate $\quad 1$-(3-methyl-2- butenoate) [7t]}

Compound 7t was prepared by using the general procedure (B) (reaction time: 1h), with commercial 3,3-dimethylacrylic acid $\mathbf{5 t}$ (19.2 mg, $0.19 \mathrm{mmol}, 1.0$ equiv.) and $\mathbf{1 a}$ (104.9 mg, 0.29 mmol, 1.5 equiv.), which afforded the product as an amorphous solid $(64.3 \mathrm{mg}, 78 \%$ yield after column chromatography EtOAc/Cyclohexane $3: 7) ; \quad$ TLC $\quad \mathbf{R}_{\mathbf{f}}=0.46$ (EtOAc/Cyclohexane, $\left.1: 1, \mathrm{SiO}_{2}\right) ;[\boldsymbol{\alpha}]_{\mathbf{D}}{ }^{\mathbf{2 0}}=-4.9(\mathrm{c}, 1.00 \mathrm{in}$ $\mathrm{CHCl}_{3}$ ); IR (neat, $\mathbf{c m}^{-1}$ ) 3487, 2964, 1757, 1646, 1434, 1367, 1212, 1081, 1035 ; ${ }^{\mathbf{1}} \mathbf{H}$ NMR (300 MHz, $\mathbf{C D C l}_{\mathbf{3}}$ ) $\boldsymbol{\delta}$ (ppm) 5.74 $(\mathrm{d}, J=8.3 \mathrm{~Hz}, 1 \mathrm{H}), 5.67(\mathrm{~s}, 1 \mathrm{H}), 5.32-5.21(\mathrm{~m}, 1 \mathrm{H}), 5.14(\mathrm{dt}$, $J=9.5,6.8 \mathrm{~Hz}, 2 \mathrm{H}), 4.28(\mathrm{dd}, J=12.5,4.4 \mathrm{~Hz}, 1 \mathrm{H}), 4.10(\mathrm{dd}$, $J=12.5,2.2 \mathrm{~Hz}, 1 \mathrm{H}), 3.84(\mathrm{ddd}, J=10.0,4.6,2.1 \mathrm{~Hz}, 1 \mathrm{H})$, $2.17(\mathrm{~s}, 3 \mathrm{H}), 2.06(\mathrm{~s}, 3 \mathrm{H}), 2.02(\mathrm{~s}, 3 \mathrm{H}), 2.00(\mathrm{~s}, 6 \mathrm{H}), 1.91(\mathrm{~s}$, $3 \mathrm{H}) ;{ }^{13} \mathbf{C}\{1 \mathrm{H}\}$ NMR (75 MHz, $\left.\mathbf{C D C l}_{3}\right) \delta$ (ppm) $170.7\left(\mathrm{C}_{\mathrm{q}}\right)$, $170.2\left(\mathrm{C}_{\mathrm{q}}\right), 169.5\left(\mathrm{C}_{\mathrm{q}}\right), 169.4\left(\mathrm{C}_{\mathrm{q}}\right), 164.1\left(\mathrm{C}_{\mathrm{q}}\right), 161.4\left(\mathrm{C}_{\mathrm{q}}\right)$, $114.7(\mathrm{CH}), 91.3(\mathrm{CH}), 73.0(\mathrm{CH}), 72.7(\mathrm{CH}), 70.4(\mathrm{CH}), 68.0$ (CH), $61.6\left(\mathrm{CH}_{2}\right), 27.8\left(\mathrm{CH}_{3}\right), 20.8\left(\mathrm{CH}_{3}\right), 20.7\left(4 \times \mathrm{CH}_{3}\right)$; HRMS (ESI) $(\mathbf{M}+\mathbf{N a})^{+} \mathrm{m} / z$ calculated for $\mathrm{C}_{19} \mathrm{H}_{26} \mathrm{O}_{11} \mathrm{Na}$ 453.1373, found 453.1378 .

\section{$\beta$-D-Glucopyranose, 2,3,4,6-tetraacetate $\quad 1$-(2- thiophenecarboxylate) [7u]}

Compound $\mathbf{7 u}$ was prepared by using both general procedures (A) (reaction time: $15 \mathrm{~min}$ ) with commercial 2-thiophenecarboxylic acid $\mathbf{5 u}(24.6 \mathrm{mg}, 0.19 \mathrm{mmol}, 1.0$ equiv.) and 1a (104.9 mg, $0.29 \mathrm{mmol}, 1.5$ equiv.), which afforded the product as a white solid (with $\mathrm{Cu}$-catalyst 0.156 mmol, $82 \%$ yield, with Co-catalyst $0.157 \mathrm{mmol}, 83 \%$ yield) after column chromatography EtOAc/Cyclohexane 3:7); TLC $\mathbf{R}_{\mathbf{f}}=0.50\left(\right.$ EtOAc/Cyclohexane, $\left.1: 1, \mathrm{SiO}_{2}\right) ; \mathbf{m p}: 115.5-116.7$ ${ }^{\circ} \mathrm{C} ;\left[\boldsymbol{\alpha}_{\mathbf{D}}{ }^{\mathbf{2 0}}=-20.8\left(\mathrm{c}, 1.00\right.\right.$ in $\mathrm{CHCl}_{3}$ ); IR (neat, $\mathbf{c m}^{-\mathbf{1}}$ ) 2959, 1758, 1524, 1416, 1364, 1221, 1065, 1037; ${ }^{\mathbf{1}} \mathbf{H}$ NMR (300 MHz, $\left.\mathbf{C D C l}_{3}\right) \boldsymbol{\delta}$ (ppm) $7.85(\mathrm{~d}, J=4.1 \mathrm{~Hz}, 1 \mathrm{H}), 7.64(\mathrm{~d}, J=$ $4.8 \mathrm{~Hz}, 1 \mathrm{H}), 7.18-7.04(\mathrm{~m}, 1 \mathrm{H}), 5.92-5.81(\mathrm{~m}, 1 \mathrm{H}), 5.36-$ $5.27(\mathrm{~m}, 2 \mathrm{H}), 5.25-5.11(\mathrm{~m}, 1 \mathrm{H}), 4.32(\mathrm{dd}, J=12.5,4.6 \mathrm{~Hz}$, $1 \mathrm{H}), 4.13$ (dd, $J=12.6,2.3 \mathrm{~Hz}, 1 \mathrm{H}), 3.93$ (ddd, $J=9.8,4.5$, $2.3 \mathrm{~Hz}, 1 \mathrm{H}), 2.07$ (s, 3H), $2.04(\mathrm{~s}, 3 \mathrm{H}), 2.03$ (s, 3H), $2.00(\mathrm{~s}$, $3 \mathrm{H}) ;{ }^{13} \mathbf{C}\{1 \mathrm{H}\}$ NMR (75 MHz, $\left.\mathbf{C D C l}_{3}\right) \delta(\mathbf{p p m}) 170.8\left(\mathrm{C}_{\mathrm{q}}\right)$, $170.2\left(\mathrm{C}_{\mathrm{q}}\right), 169.6\left(\mathrm{C}_{\mathrm{q}}\right), 169.4\left(\mathrm{C}_{\mathrm{q}}\right), 160.1\left(\mathrm{C}_{\mathrm{q}}\right), 135.3(\mathrm{CH})$, $134.4(\mathrm{CH}), 131.9\left(\mathrm{C}_{\mathrm{q}}\right), 128.3(\mathrm{CH}), 92.5(\mathrm{CH}), 72.9(\mathrm{CH})$, $72.7(\mathrm{CH}), 70.2(\mathrm{CH}), 68.0(\mathrm{CH}), 61.6\left(\mathrm{CH}_{2}\right), 20.8\left(\mathrm{CH}_{3}\right), 20.7$
(3 $\left.\mathrm{x} \mathrm{CH}_{3}\right)$; HRMS (ESI) $(\mathbf{M}+\mathbf{N a})^{+} \mathrm{m} / \mathrm{z}$ calculated for $\mathrm{C}_{19} \mathrm{H}_{22} \mathrm{O}_{11} \mathrm{SNa} 481.0781$, found 481.0783.

$\beta$-D-Glucopyranose, 2,3,4,6-tetraacetate $\quad 1$-(3quinolinecarboxylate) [7v]

Compound $\mathbf{7 v}$ was prepared by using the general procedure (B) (reaction time: 1h), with commercial 3-quinolinecarboxylic acid $\mathbf{5 v}(33.3 \mathrm{mg}, 0.19 \mathrm{mmol}, 1.0$ equiv.) and $1 \mathrm{a}$ (104.9 $\mathrm{mg}, 0.29 \mathrm{mmol}, 1.5$ equiv.), which afforded the product as a white solid $(48.2 \mathrm{mg}, 50 \%$ yield after column chromatography EtOAc/Cyclohexane 3:7); TLC $\mathbf{R}_{\mathbf{f}}=$ 0.27 (EtOAc/Cyclohexane, $1: 1, \mathrm{SiO}_{2}$ ); $\mathbf{m p}: 190.0-192.2{ }^{\circ} \mathrm{C}$; $[\boldsymbol{\alpha}]_{\mathbf{D}}{ }^{\mathbf{2 0}}=-23.9\left(\mathrm{c}, 1.00\right.$ in $\left.\mathrm{CHCl}_{3}\right)$; IR (neat, $\mathbf{~ c m}^{-\mathbf{1}}$ ) 3492, 2959, 1757, 1621, 1367, 1213, 1077, 1034; ${ }^{\mathbf{1}} \mathbf{H}$ NMR (300 MHz, $\left.\mathbf{C D C l}_{3}\right) \boldsymbol{\delta}(\mathbf{p p m}) 9.42(\mathrm{~d}, J=2.3 \mathrm{~Hz}, 1 \mathrm{H}), 8.84(\mathrm{~d}, J=2.4 \mathrm{~Hz}$, $1 \mathrm{H}), 8.16(\mathrm{~d}, J=8.5 \mathrm{~Hz}, 1 \mathrm{H}), 7.96(\mathrm{~d}, J=7.9 \mathrm{~Hz}, 1 \mathrm{H}), 7.92-$ $7.81(\mathrm{~m}, 1 \mathrm{H}), 7.64(\mathrm{t}, J=7.5 \mathrm{~Hz}, 1 \mathrm{H}), 6.01(\mathrm{dd}, J=5.3,2.6$ $\mathrm{Hz}, 1 \mathrm{H}), 5.44-5.31(\mathrm{~m}, 2 \mathrm{H}), 5.22$ (ddd, $J=9.3,6.2,2.9 \mathrm{~Hz}$, $1 \mathrm{H}), 4.34(\mathrm{dd}, J=12.6,4.5 \mathrm{~Hz}, 1 \mathrm{H}), 4.15(\mathrm{dd}, J=12.5,2.4$ Hz, 1H), 3.98 (ddd, $J=10.1,4.6,2.3 \mathrm{~Hz}, 1 \mathrm{H}), 2.07$ (s, 3H), $2.05(\mathrm{~s}, 3 \mathrm{H}), 2.04(\mathrm{~s}, 3 \mathrm{H}), 1.98(\mathrm{~s}, 3 \mathrm{H}) ;{ }^{13} \mathbf{C}\{\mathbf{1 H}\}$ NMR (75 MHz, $\left.\mathbf{C D C l}_{3}\right) \boldsymbol{\delta}$ (ppm) $170.7\left(\mathrm{C}_{\mathrm{q}}\right), 170.2\left(\mathrm{C}_{\mathrm{q}}\right), 169.5\left(\mathrm{C}_{\mathrm{q}}\right)$, $169.4\left(\mathrm{C}_{\mathrm{q}}\right), 163.6\left(\mathrm{C}_{\mathrm{q}}\right), 150.3\left(\mathrm{C}_{\mathrm{q}}\right), 150.1(\mathrm{CH}), 139.8(\mathrm{CH})$, $132.7(\mathrm{CH}), 129.7(\mathrm{CH}), 129.5(\mathrm{CH}), 127.9(\mathrm{CH}), 126.8\left(\mathrm{C}_{\mathrm{q}}\right)$, $121.4\left(\mathrm{C}_{\mathrm{q}}\right), 92.7(\mathrm{CH}), 73.0(\mathrm{CH}), 72.7(\mathrm{CH}), 70.3(\mathrm{CH}), 67.9$ $(\mathrm{CH}), 61.6\left(\mathrm{CH}_{2}\right), 20.8\left(\mathrm{CH}_{3}\right), 20.7\left(3 \times \mathrm{CH}_{3}\right)$; HRMS (ESI) $(\mathbf{M}+\mathbf{N a})^{+} \mathrm{m} / z$ calculated for $\mathrm{C}_{24} \mathrm{H}_{25} \mathrm{NO}_{11} \mathrm{Na} 526.1320$, found 526.1328 .

\section{$\beta$-D-Glucopyranose, 2,3,4,6-tetraacetate $\quad 1-(3-$ benzofurancarboxylate) [7w]}

Compound $\mathbf{7 w}$ was prepared by using the general procedure $\mathbf{A}$ (reaction time: $15 \mathrm{~min}$ ), with commercial benzofuran-3carboxylic acid $\mathbf{5 w}(31.1 \mathrm{mg}, 0.19 \mathrm{mmol}, 1.0$ equiv.) and 1a (104.9 mg, $0.29 \mathrm{mmol}, 1.5$ equiv.), which afforded the product as a white solid $(0.114 \mathrm{mmol}, 60 \%$ yield after column chromatography EtOAc/Cyclohexane 4:6); $\quad \mathbf{R}_{\mathbf{f}} \quad(40 \%$ EtOAc/Cyclohexane $)=0.8 ; \mathbf{m p ~} 122-128{ }^{\circ} \mathrm{C} ;[\boldsymbol{\alpha}]_{\mathbf{D}}{ }^{25}=-58.33$ (c 0,006 $\mathrm{CH}_{2} \mathrm{Cl}_{2}$ ); IR (neat, $\mathbf{c m}^{-1}$ ) 2923, 2361, 1738, 1260, 1221, 1072, 1035, 797; ${ }^{\mathbf{1}} \mathbf{H}$ NMR (300 MHz, $\left.\mathbf{C D C l}_{\mathbf{3}}\right) \delta$ (ppm) $8.30(\mathrm{~s}, 1 \mathrm{H}), 8.07-8.00(\mathrm{~m}, J=6.0,3.1 \mathrm{~Hz}, 1 \mathrm{H}), 7.56-7.52$ $(\mathrm{m}, J=6.1,3.1 \mathrm{~Hz}, 1 \mathrm{H}), 7.40-7.35(\mathrm{~m}, J=6.0,3.2 \mathrm{~Hz}, 2 \mathrm{H})$, $5.95(\mathrm{dd}, J=5.8,2.3 \mathrm{~Hz}, 1 \mathrm{H}), 5.35(\mathrm{dd}, J=5.9,2.8 \mathrm{~Hz}, 2 \mathrm{H})$, $5.25-5.16(\mathrm{~m}, J=9.6,6.5,3.0 \mathrm{~Hz}, 1 \mathrm{H}), 4.34(\mathrm{dd}, J=12.5$, $4.4 \mathrm{~Hz}, 1 \mathrm{H}), 4.14(\mathrm{dd}, J=12.5,2.0 \mathrm{~Hz}, 1 \mathrm{H}), 4.00-3.92(\mathrm{~m}$, $1 \mathrm{H}), 2.07(\mathrm{~s}, 3 \mathrm{H}), 2.04(\mathrm{~d}, J=2.2 \mathrm{~Hz}, 6 \mathrm{H}), 1.99(\mathrm{~s}, 3 \mathrm{H})$; ${ }^{13} \mathbf{C}\{1 \mathbf{H}\}$ NMR (75 $\left.\mathbf{M H z}, \mathbf{C D C l}_{3}\right) \delta(\mathbf{p p m}) \quad 170.59\left(\mathrm{C}_{\mathrm{q}}\right)$, $170.06\left(\mathrm{C}_{\mathrm{q}}\right), 169.41\left(\mathrm{C}_{\mathrm{q}}\right), 169.36\left(\mathrm{C}_{\mathrm{q}}\right), 160.94\left(\mathrm{C}_{\mathrm{q}}\right), 155.63\left(\mathrm{C}_{\mathrm{q}}\right)$, 152.57(CH), $125.68(\mathrm{CH}), \quad 124.58(\mathrm{CH}), \quad 124.07\left(\mathrm{C}_{\mathrm{q}}\right)$, 121.84(CH), 113.01( $\left(\mathrm{C}_{\mathrm{q}}\right), 111.80(\mathrm{CH}), 91.85(\mathrm{CH}), 72.77(\mathrm{CH})$, 72.62(CH), 70.11(CH), 67.87(CH), 61.44( $\left(\mathrm{CH}_{2}\right), 20.69\left(\mathrm{CH}_{3}\right)$, $20.58\left(3 \times \mathrm{CH}_{3}\right)$; HRMS (ESI) $(\mathbf{M}+\mathbf{N a})^{+} \mathrm{m} / \mathrm{z}$ calculated for $\mathrm{C}_{23} \mathrm{H}_{24} \mathrm{O}_{12} \mathrm{Na}$ : 515.1165, found 515.1167.

$\beta$-D-Glucopyranose, $\quad$ 2,3,4,6-tetraacetate $\quad 1$ tridecancarboxylate) [7x]

Compound 7x was prepared by using both general procedures (A) (reaction time: $15 \mathrm{~min}$ ), and (B) (reaction time: $1 \mathrm{~h}$ ), with commercial tridecanoic acid $\mathbf{5 x}(41.2 \mathrm{mg}, 0.19 \mathrm{mmol}, 1.0$ equiv.) and $1 \mathrm{a}$ (104.9 $\mathrm{mg}, 0.29 \mathrm{mmol}, 1.5$ equiv.), which afforded the product as a transparent oil (with $\mathrm{Cu}$-catalyst 0.114 mmol, $65 \%$ yield, with Co-catalyst $0.15 \mathrm{mmol}, 79 \%$ yield) 
after column chromatography EtOAc/Cyclohexane 3:7); TLC $\mathbf{R}_{\mathbf{f}}=0.46\left(\right.$ EtOAc/Cyclohexane, $\left.1: 1, \mathrm{SiO}_{2}\right) ;[\boldsymbol{\alpha}]_{\mathbf{D}}{ }^{20}=-3.5(\mathrm{c}$, 1.00 in $\mathrm{CHCl}_{3}$ ); IR (neat, $\mathbf{~ c m}^{-1}$ ) 2924, 2854, 1758, 1468, 1366, 1208, 1164, 1082, 1068 ; ${ }^{\mathbf{1}} \mathbf{H}$ NMR (300 $\left.\mathbf{M H z}, \mathbf{C D C l}_{3}\right) \delta$ (ppm) $5.72(\mathrm{~d}, J=8.3 \mathrm{~Hz}, 1 \mathrm{H}), 5.23(\mathrm{~d}, J=9.3 \mathrm{~Hz}, 1 \mathrm{H}), 5.20$ $-5.04(\mathrm{~m}, 2 \mathrm{H}), 4.29(\mathrm{dd}, J=12.5,4.4 \mathrm{~Hz}, 1 \mathrm{H}), 4.09(\mathrm{dd}, J=$ $12.5,2.2 \mathrm{~Hz}, 1 \mathrm{H}), 3.83$ (ddd, $J=9.9,4.6,2.1 \mathrm{~Hz}, 1 \mathrm{H}), 2.34(\mathrm{t}$, $J=7.5 \mathrm{~Hz}, 2 \mathrm{H}), 2.07(\mathrm{~s}, 3 \mathrm{H}), 2.02(\mathrm{~s}, 3 \mathrm{H}), 2.01(\mathrm{~s}, 3 \mathrm{H}), 2.00$ $(\mathrm{s}, 3 \mathrm{H}), 1.66-1.51(\mathrm{~m}, 2 \mathrm{H}), 1.30(\mathrm{~d}, J=7.0 \mathrm{~Hz}, 2 \mathrm{H}), 1.27(\mathrm{~s}$, $2 \mathrm{H}), 1.24(\mathrm{~s}, 14 \mathrm{H}), 0.86(\mathrm{t}, J=6.7 \mathrm{~Hz}, 3 \mathrm{H}) ;{ }^{13} \mathbf{C}\{\mathbf{1 H}\} \mathbf{N M R}$ (75 MHz, $\left.\mathbf{C D C l}_{3}\right) \boldsymbol{\delta}$ (ppm) $171.9\left(\mathrm{C}_{\mathrm{q}}\right), 170.7\left(\mathrm{C}_{\mathrm{q}}\right), 170.2\left(\mathrm{C}_{\mathrm{q}}\right)$, $169.5\left(\mathrm{C}_{\mathrm{q}}\right), 169.3\left(\mathrm{C}_{\mathrm{q}}\right), 91.7(\mathrm{CH}), 72.9(\mathrm{CH}), 72.8(\mathrm{CH}), 70.3$ $(\mathrm{CH}), 67.9(\mathrm{CH}), 61.6\left(\mathrm{CH}_{2}\right), 34.2\left(\mathrm{CH}_{2}\right), 32.0\left(\mathrm{CH}_{2}\right), 29.7(3$ x $\left.\mathrm{CH}_{2}\right), 29.5\left(2 \times \mathrm{CH}_{2}\right), 29.3\left(\mathrm{CH}_{2}\right), 29.1\left(\mathrm{CH}_{2}\right), 24.7\left(\mathrm{CH}_{2}\right)$, $22.8\left(\mathrm{CH}_{2}\right), 20.8\left(\mathrm{CH}_{3}\right), 20.7\left(3 \times \mathrm{CH}_{3}\right), 14.2\left(\mathrm{CH}_{3}\right) ;$ HRMS (ESI) $(\mathbf{M}+\mathbf{N a})^{+} \mathrm{m} / z$ calculated for $\mathrm{C}_{27} \mathrm{H}_{44} \mathrm{O}_{11} \mathrm{Na}$ 567.2781, found 567.2797.

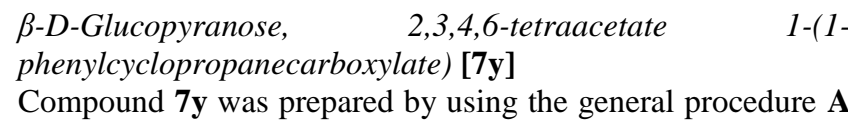
(reaction time: $15 \mathrm{~min}$ ), with commercial 1phenylcyclopropane-1-carboxylic acid $\mathbf{5 y} \quad(31.2 \mathrm{mg}, \quad 0.19$ mmol, 1.0 equiv.) and $1 \mathrm{a}$ (104.9 $\mathrm{mg}, 0.29 \mathrm{mmol}, 1.5$ equiv.), which afforded the product as a white solid (52 $\mathrm{mg}, 55 \%$ yield after column chromatography EtOAc/Cyclohexane 4:6); $\mathbf{R}_{\mathbf{f}}$ $(40 \%$ EtOAc/Cyclohexane $)=0.54 ; \mathbf{~ m p ~} 154-162{ }^{\circ} \mathrm{C} ;[\boldsymbol{\alpha}]_{\mathbf{D}}{ }^{25}=$ -13.33 (c $0,006 \mathrm{CH}_{2} \mathrm{Cl}_{2}$ ); IR (neat, $\mathbf{c m}^{-1}$ ) 3250, 1754, 1742, 1629, 1252, 1231, 1082; ${ }^{\mathbf{1}} \mathbf{H}$ NMR (300 $\left.\mathbf{~ M H z ,} \mathbf{C D C l}_{3}\right) \delta$ (ppm) $7.29(\mathrm{~s}, 5 \mathrm{H}), 5.61(\mathrm{~d}, \mathrm{~J}=8.1 \mathrm{~Hz}, 1 \mathrm{H}), 5.17(\mathrm{~d}, \mathrm{~J}=9.2$ $\mathrm{Hz}, 1 \mathrm{H}), 5.07$ (t, J = 9.6 Hz, 1H), 4.98 (t, J = 8.7 Hz, 1H), 4.26 $(\mathrm{dd}, \mathrm{J}=12.5,4.4 \mathrm{~Hz}, 1 \mathrm{H}), 4.12-4.04(\mathrm{~m}, \mathrm{~J}=17.0,4.5 \mathrm{~Hz}$, $1 \mathrm{H}), 3.83-3.77(\mathrm{~m}, 1 \mathrm{H}), 2.07(\mathrm{~s}, 3 \mathrm{H}), 2.00(\mathrm{~s}, 3 \mathrm{H}), 1.98(\mathrm{~s}$, $3 \mathrm{H}), 1.96(\mathrm{~s}, 3 \mathrm{H}), 1.77-1.69(\mathrm{~m}, 1 \mathrm{H}), 1.60-1.52(\mathrm{~m}, 1 \mathrm{H})$, $1.26(\mathrm{~d}, \mathrm{~J}=7.1 \mathrm{~Hz}, 2 \mathrm{H}) ;{ }^{13} \mathbf{C}\{\mathbf{1 H}\} \mathbf{N M R}\left(\mathbf{7 5} \mathbf{M H z}, \mathbf{C D C l}_{3}\right)$ $\boldsymbol{\delta}(\mathbf{p p m}) \quad 172.60\left(\mathrm{C}_{\mathrm{q}}\right), \quad 170.60\left(\mathrm{C}_{\mathrm{q}}\right), \quad 170.06\left(\mathrm{C}_{\mathrm{q}}\right), \quad 169.33\left(\mathrm{C}_{\mathrm{q}}\right)$, 168.82 $\left(\mathrm{C}_{\mathrm{q}}\right), 138.23\left(\mathrm{C}_{\mathrm{q}}\right), 130.4(2 \times \mathrm{CH}), 128.24(2 \times \mathrm{CH})$, 127.54(CH), 92.12(CH), 72.67(CH), 72.62(CH), 69.96(CH), 67.77( $(\mathrm{CH}), \quad 61.45\left(\mathrm{CH}_{2}\right), \quad 28.94\left(\mathrm{C}_{\mathrm{q}}\right), 20.72\left(\mathrm{CH}_{3}\right), \quad 20.54(2 \mathrm{x}$ $\left.\mathrm{CH}_{3}\right), 20.46\left(\mathrm{CH}_{3}\right), 17.00\left(\mathrm{CH}_{2}\right), 16.96\left(\mathrm{CH}_{2}\right) ;$ HRMS (ESI) (M $+\mathrm{Na})^{+} \mathrm{m} / \mathrm{z}$ calculated for $\mathrm{C}_{24} \mathrm{H}_{28} \mathrm{O}_{11} \mathrm{Na}$ : 515.1524, found 515.1523 .

\section{$\beta$-D-Glucopyranose, $\quad 2,3,4,6$-tetraacetate $\quad 1-(1,2,3,4$ - tetrahydro-1-naphthalenecarboxylate] [7z]}

Compound $\mathbf{7 z}$ was prepared by using the general procedure (B) (reaction time: 1h), with commercial 1,2,3,4-tetrahydronaphthalene-1-carboxylic acid $\mathbf{5 z}(33.8 \mathrm{mg}$, 0.19 mmol, 1.0 equiv.) and 1a (104.9 mg, $0.29 \mathrm{mmol}, 1.5$ equiv.), which afforded the product as a white solid $(65.7 \mathrm{mg}$, $68 \%$ yield after column chromatography EtOAc/Cyclohexane $3: 7)$; diastereomeric mixture mostly visible on ${ }^{13} \mathrm{C}$ NMR spectra; TLC $\mathbf{R}_{\mathbf{f}}=0.53\left(\right.$ EtOAc/Cyclohexane, 1:1, $\left.\mathrm{SiO}_{2}\right)$; $\mathbf{m p}$ : $101.0-102.4{ }^{\circ} \mathrm{C} ;[\mathbf{\alpha}]_{\mathbf{D}}{ }^{\mathbf{2 0}}=-9.1\left(\mathrm{c}, 1.00\right.$ in $\left.\mathrm{CHCl}_{3}\right)$; IR (neat, $\mathbf{c m}^{-1}$ ) 2944, 1757, 1455, 1366, 1209, 1073, 1033; ${ }^{\mathbf{1}} \mathbf{H}$ NMR $\left(300 \mathbf{M H z} \mathbf{C D C l}_{3}\right) \delta$ (ppm) $7.21-7.01(\mathrm{~m}, 4 \mathrm{H}), 5.73(\mathrm{dd}, J=$ 7.5, $5.2 \mathrm{~Hz}, 1 \mathrm{H}), 5.30-5.02(\mathrm{~m}, 3 \mathrm{H}), 4.36-4.22(\mathrm{~m}, 1 \mathrm{H})$, $4.11(\mathrm{dd}, J=12.6,1.3 \mathrm{~Hz}, 1 \mathrm{H}), 3.95-3.69(\mathrm{~m}, 2 \mathrm{H}), 2.91-$ $2.61(\mathrm{~m}, 2 \mathrm{H}), 2.24-2.12(\mathrm{~m}, 1 \mathrm{H}), 2.08(\mathrm{~s}, 3 \mathrm{H}), 2.02(\mathrm{~s}, 3 \mathrm{H})$, $2.00(\mathrm{~d}, J=1.5 \mathrm{~Hz}, 3 \mathrm{H}), 1.96-1.88(\mathrm{~m}, 1 \mathrm{H}), 1.86(\mathrm{~d}, J=3.9$ $\mathrm{Hz}, 3 \mathrm{H}), 1.83-1.68(\mathrm{~m}, 2 \mathrm{H}) ;{ }^{13} \mathbf{C}\{\mathbf{1 H}\}$ NMR (75 MHz, $\left.\mathbf{C D C l}_{3}\right) \boldsymbol{\delta}(\mathbf{p p m}) 173.0\left(\mathrm{C}_{\mathrm{q}}\right), 172.7\left(\mathrm{C}_{\mathrm{q}}\right), 170.7\left(\mathrm{C}_{\mathrm{q}}\right), 170.2$ $\left(\mathrm{C}_{\mathrm{q}}\right), 169.5\left(\mathrm{C}_{\mathrm{q}}\right.$, which is splited into 169.3$), 137.6\left(\mathrm{C}_{\mathrm{q}}\right.$, which is splited into 137.4), $132.13\left(\mathrm{C}_{\mathrm{q}}\right), 129.6(2 \times \mathrm{CH}$, which is splited into 129.5 and 129.4), $127.25(\mathrm{CH}), 126.0(\mathrm{CH}$, which is splited into 125.9), $92.0(\mathrm{CH}$, which is splited into 91.8$)$, 72.9 ( $2 \times \mathrm{CH}$, which is splited into 73.0 and 72.8), $70.2(\mathrm{CH}$, which is splited into 70.1), $68.0(\mathrm{CH}$, which is splited into 67.9), $61.6\left(\mathrm{CH}_{2}\right), 44.8(\mathrm{CH}$, which is splited into 43.9), 29.1 $\left(\mathrm{CH}_{2}\right.$, which is splited into 29.0$), 26.5\left(\mathrm{CH}_{2}\right.$, which is splited into 25.8), $20.9\left(\mathrm{CH}_{3}\right.$, which is splited into 20.4), $20.7(3 \mathrm{x}$ $\left.\mathrm{CH}_{3}\right), 20.2\left(\mathrm{CH}_{2}\right) ; \mathbf{H R M S}(\mathbf{E S I})(\mathbf{M}+\mathbf{N a})^{+} \mathrm{m} / z$ calculated for $\mathrm{C}_{25} \mathrm{H}_{30} \mathrm{O}_{11} \mathrm{Na}$ 529.1680, found 529.1682.

D-glycero-D-gulo-Octonic acid, 3,7-anhydro-2-deoxy-, 2,3,4, 6-tetra-O-acetyl- $\beta$-D-glucopyranosyl ester, 4,5,6,8tetraacetate [7aa]

Compound 7aa was prepared by using the general procedure A (reaction time: $15 \mathrm{~min})$, with $2-((2 \mathrm{~S}, 3 \mathrm{~S}, 4 \mathrm{R}, 5 \mathrm{R}, 6 \mathrm{R})-3,4,5-$ triacetoxy-6-(acetoxymethyl)tetrahydro-2H-pyran-2-yl)acetic acid 5aa (75 mg, 0.19 mmol, 1.0 equiv.) and 1a (104.9 mg, 0.29 mmol, 1.5 equiv.), which afforded the product as a white solid $(79 \mathrm{mg}, 60 \%$ yield after column chromatography EtOAc/Cyclohexane 4:6); $\mathbf{R}_{\mathbf{f}}(50 \%$ EtOAc/Cyclohexane $)=$ 0.28 ; $\mathbf{m p} 155-162{ }^{\circ} \mathrm{C} ;[\mathbf{\alpha}]_{\mathbf{D}}{ }^{25}=-90.00\left(\mathrm{c} 0,002 \mathrm{CH}_{2} \mathrm{Cl}_{2}\right)$; IR (neat, $\mathbf{~ c m}^{-1}$ ) 1742, 1704, 1365, 1258, 1227, 1149, 1032; ${ }^{\mathbf{1}} \mathbf{H}$

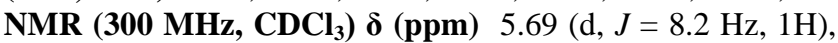
$5.25(\mathrm{~d}, J=9.4 \mathrm{~Hz}, 1 \mathrm{H}), 5.16-5.04(\mathrm{~m}, J=15.3,8.9 \mathrm{~Hz}, 4 \mathrm{H})$, $4.91(\mathrm{t}, J=9.6 \mathrm{~Hz}, 1 \mathrm{H}), 4.30-4.19(\mathrm{~m}, 2 \mathrm{H}), 4.11-4.06(\mathrm{~m}, J$ $=7.8,3.5 \mathrm{~Hz}, 1 \mathrm{H}), 3.96-3.88(\mathrm{~m}, 1 \mathrm{H}), 3.87-3.81(\mathrm{~m}, J=$ 9.0, 3.1 Hz, 1H), $3.74-3.70(\mathrm{~m}, 1 \mathrm{H}), 3.70-3.66(\mathrm{~m}, 1 \mathrm{H})$, $3.64-3.59(\mathrm{~m}, J=10.1 \mathrm{~Hz}, 1 \mathrm{H}), 3.49-3.45(\mathrm{~m}, J=8.1,5.3$ $\mathrm{Hz}, 1 \mathrm{H}), 2.07$ (s, 3H), 2.07 (s, 3H), 2.05 (s, 3H), 2.02 (d, $J=$ $3.4 \mathrm{~Hz}, 9 \mathrm{H}), 2.01(\mathrm{~s}, 3 \mathrm{H}), 1.99(\mathrm{~s}, 3 \mathrm{H}) ;{ }^{13} \mathbf{C}\{\mathbf{1 H}\} \mathbf{N M R}(\mathbf{7 5}$ MHz, $\left.\mathbf{C D C l}_{3}\right) \delta$ (ppm) $170.51\left(\mathrm{C}_{\mathrm{q}}\right), 170.23\left(\mathrm{C}_{\mathrm{q}}\right), 170.03\left(\mathrm{C}_{\mathrm{q}}\right)$, 169.41 $\left(\mathrm{C}_{\mathrm{q}}\right), 169.34\left(\mathrm{C}_{\mathrm{q}}\right), 167.95\left(\mathrm{C}_{\mathrm{q}}\right), 91.91(\mathrm{CH}), 75.95(\mathrm{CH})$, 73.96(CH), 73.83(CH), 72.81(CH), 72.53(CH), 71.50(CH), $71.27\left(\mathrm{C}_{\mathrm{q}}\right), \quad 70.50\left(\mathrm{C}_{\mathrm{q}}\right), \quad 70.17\left(\mathrm{C}_{\mathrm{q}}\right), \quad 70.00(\mathrm{CH}), \quad 68.25(\mathrm{CH})$, 67.71(CH), 61.89( $\left(\mathrm{CH}_{2}\right), 61.35\left(\mathrm{CH}_{2}\right), 37.17\left(\mathrm{CH}_{2}\right), 20.67(3 \mathrm{x}$ $\left.\mathrm{CH}_{3}\right), 20.54\left(5 \times \mathrm{CH}_{3}\right) ;$ HRMS (ESI) $(\mathbf{M}+\mathbf{N a})^{+} \mathrm{m} / z$ calculated for $\mathrm{C}_{30} \mathrm{H}_{40} \mathrm{O}_{20} \mathrm{Na}$ : 743.2011, found 743.1990.

2.1 Late stage functionalization of pharmaceutical carboxylates

Procedure for the synthesis of $\beta$-D-Glucopyranose, $2,3,4,6$ tetraacetate 1-[3-(aminosulfonyl)-5-(butylamino)-4phenoxybenzoate] [7ab] and its characterization data

Compound 7ab was prepared by using the general procedure (reaction time: 2h), with commercial Bumetanide $(70.0 \mathrm{mg}$, $0.19 \mathrm{mmol}, 1.0$ equiv.) and $\mathbf{1 a}(104.9 \mathrm{mg}, 0.29 \mathrm{mmol}, 1.5$ equiv.), which afforded the product as a white solid $(44.1 \mathrm{mg}$, $33 \%$ yield after column chromatography EtOAc/Cyclohexane $2: 8$-> 3:7); $\mathbf{T L C} \mathbf{R}_{\mathbf{f}}=0.35\left(\right.$ EtOAc/Cyclohexane, 1:1, $\left.\mathrm{SiO}_{2}\right)$; mp: $103.4-104.5^{\circ} \mathrm{C} ;[\mathbf{\alpha}]_{\mathbf{D}}{ }^{20}=-15.1\left(\mathrm{c}, 1.00\right.$ in $\left.\mathrm{CHCl}_{3}\right)$; IR (neat, $\mathbf{c m}^{-1}$ ) 3418, 3270, 2959, 2924, 1745, 1436, 1367, 1213, 1034; ${ }^{1} \mathbf{H}$ NMR (300 MHz, $\mathbf{C D C l}_{3}$ ) $\boldsymbol{\delta}$ (ppm) $7.95(\mathrm{~d}, J=1.8$ $\mathrm{Hz}, 1 \mathrm{H}), 7.51$ (d, $J=1.9 \mathrm{~Hz}, 1 \mathrm{H}), 7.39-7.23(\mathrm{~m}, 2 \mathrm{H}), 7.10(\mathrm{t}$, $J=7.3 \mathrm{~Hz}, 1 \mathrm{H}), 6.91(\mathrm{dd}, J=8.6,2.9 \mathrm{~Hz}, 2 \mathrm{H}), 5.96-5.84(\mathrm{~m}$, $1 \mathrm{H}), 5.39-5.27(\mathrm{~m}, 2 \mathrm{H}), 5.27-5.12(\mathrm{~m}, 1 \mathrm{H}), 5.06(\mathrm{~s}, 2 \mathrm{H})$, $4.32(\mathrm{dd}, J=12.5,4.5 \mathrm{~Hz}, 1 \mathrm{H}), 4.14(\mathrm{dd}, J=12.4,2.5 \mathrm{~Hz}$, $1 \mathrm{H}), 3.95$ (ddd, $J=12.8,7.6,3.9 \mathrm{~Hz}, 2 \mathrm{H}), 3.10(\mathrm{dd}, J=12.5$, $6.8 \mathrm{~Hz}, 2 \mathrm{H}), 2.07(\mathrm{~s}, 3 \mathrm{H}), 2.05(\mathrm{~s}, 3 \mathrm{H}), 2.04(\mathrm{~s}, 3 \mathrm{H}), 2.02(\mathrm{~s}$, $3 \mathrm{H}), 1.73-1.56(\mathrm{~m}, 1 \mathrm{H}), 1.41-1.27(\mathrm{~m}, 1 \mathrm{H}), 1.22-1.07(\mathrm{~m}$, 
2H), $0.82(\mathrm{t}, J=7.3 \mathrm{~Hz}, 3 \mathrm{H}) ;{ }^{13} \mathbf{C}\{\mathbf{1 H}\}$ NMR (75 MHz, $\left.\mathbf{C D C l}_{3}\right) \boldsymbol{\delta}(\mathbf{p p m}) 170.8\left(\mathrm{C}_{\mathrm{q}}\right), 170.2\left(\mathrm{C}_{\mathrm{q}}\right), 169.6\left(\mathrm{C}_{\mathrm{q}}\right), 169.5$ $\left(\mathrm{C}_{\mathrm{q}}\right), 163.5\left(\mathrm{C}_{\mathrm{q}}\right), 155.7\left(\mathrm{C}_{\mathrm{q}}\right), 142.5\left(\mathrm{C}_{\mathrm{q}}\right), 140.7\left(\mathrm{C}_{\mathrm{q}}\right), 136.6\left(\mathrm{C}_{\mathrm{q}}\right)$, $130.2(2 \times \mathrm{CH}), 126.4\left(\mathrm{C}_{\mathrm{q}}\right), 124.0(\mathrm{CH}), 117.1(\mathrm{CH}), 116.8$ $(\mathrm{CH}), 115.5(2 \times \mathrm{CH}), 92.8(\mathrm{CH}), 72.9(\mathrm{CH}), 72.6(\mathrm{CH}), 70.3$ $(\mathrm{CH}), 68.0(\mathrm{CH}), 61.6\left(\mathrm{CH}_{2}\right), 43.1\left(\mathrm{CH}_{2}\right), 31.0\left(\mathrm{CH}_{2}\right), 20.8$ $\left(\mathrm{CH}_{3}\right), 20.7\left(3 \times \mathrm{CH}_{3}\right), 19.9\left(\mathrm{CH}_{2}\right), 13.8\left(\mathrm{CH}_{3}\right)$; HRMS (ESI) $(\mathbf{M}+\mathbf{N a})^{+} \mathrm{m} / \mathrm{z}$ calculated for $\mathrm{C}_{31} \mathrm{H}_{38} \mathrm{~N}_{2} \mathrm{O}_{14} \mathrm{SNa}$ 717.1936, found 717.1982 .

Procedure for the synthesis of $\beta$-D-Glucopyranose, 2,3,4,6tetraacetate 1-[4-[(dipropylamino)sulfonyl]benzoate] [7ac] and its characterization data

Prepared according to the general procedure $\mathrm{C}$, thiosugar 1a (96 mg, $0.262 \mathrm{mmol}), 4-(\mathrm{N}, \mathrm{N}$-dipropylsulfamoyl)benzoic acid (Probenecid) $(50 \mathrm{mg}, 0.175 \mathrm{mmol}), \mathrm{Cu}(\mathrm{acac})_{2}(4.5 \mathrm{mg}, 5 \%$, $0.009 \mathrm{mmol})$ and $\mathrm{Ag}_{2} \mathrm{CO}_{3}(145 \mathrm{mg}, 0.525 \mathrm{mmol})$. The mixture was stirred at $130{ }^{\circ} \mathrm{C}$ for $15 \mathrm{mn}$ in microwaves. The crude product was purified by silica gel chromatography $(50 \%$ EtOAc/Pentane), and the product was isolated as a white oil $(57.15 \mathrm{mg}, 53 \%)$.

$\mathbf{R}_{\mathbf{f}}(50 \%$ EtOAc/Pentane $)=0.62 ;[\boldsymbol{\alpha}]_{\mathbf{D}}{ }^{25}=-20.1(\mathrm{c} 0.0016$ $\mathrm{CH}_{2} \mathrm{Cl}_{2}$ ) ; IR (neat, $\mathbf{c m}^{-1}$ ) 1760, 1367, 1243, 1085, $1069 ;{ }^{1} \mathbf{H}$ NMR (300 MHz, CDCl $\left.)_{3}\right) \boldsymbol{\delta}(\mathbf{p p m}) 8.14(\mathrm{~d}, J=8.3 \mathrm{~Hz}, 2 \mathrm{H})$, $7.88(\mathrm{~d}, J=8.3 \mathrm{~Hz}, 2 \mathrm{H}), 5.94-5.89(\mathrm{~m}, 1 \mathrm{H}), 5.36-5.32(\mathrm{~m}$, $2 \mathrm{H}), 5.22-5.14(\mathrm{~m}, 1 \mathrm{H}), 4.31(\mathrm{dd}, J=12.5,4.5 \mathrm{~Hz}, 1 \mathrm{H}), 4.13$ (dd, $J=12.5,1.8 \mathrm{~Hz}, 1 \mathrm{H}), 4.00-3.90(\mathrm{~m}, 1 \mathrm{H}), 3.13-3.05$ $(\mathrm{m}, 4 \mathrm{H}), 2.04(\mathrm{t}, 9 \mathrm{H}), 1.99(\mathrm{~s}, 3 \mathrm{H}), 1.61-1.46(\mathrm{~m}, J=14.8$, $7.4 \mathrm{~Hz}, 4 \mathrm{H}), 0.86(\mathrm{t}, J=7.4 \mathrm{~Hz}, 6 \mathrm{H}) ;{ }^{\mathbf{1 3}} \mathbf{C}\{\mathbf{1 H}\}$ NMR $(\mathbf{7 5}$ MHz, DMSO) $\boldsymbol{\delta}(\mathbf{p p m}) \quad 170.45\left(\mathrm{C}_{\mathrm{q}}\right), 169.95\left(\mathrm{C}_{\mathrm{q}}\right), 169.34\left(\mathrm{C}_{\mathrm{q}}\right)$, 169.27( $\left(\mathrm{C}_{\mathrm{q}}\right), \quad 163.23\left(\mathrm{C}_{\mathrm{q}}\right), \quad 145.36\left(\mathrm{C}_{\mathrm{q}}\right), \quad 131.66\left(\mathrm{C}_{\mathrm{q}}\right)$,

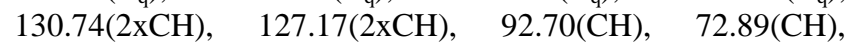
72.48( $\mathrm{CH}), \quad 70.13(\mathrm{CH}), \quad 67.85(\mathrm{CH}), \quad 61.40\left(\mathrm{CH}_{2}\right)$, $49.90\left(2 \mathrm{xCH}_{2}\right), \quad 21.89\left(2 \mathrm{xCH}_{2}\right), \quad 20.61\left(\mathrm{CH}_{3}\right), \quad 20.51\left(3 \mathrm{xCH}_{3}\right)$, $11.09\left(2 \mathrm{xCH}_{3}\right)$; HRMS (ESI) $(\mathbf{M}+\mathbf{H})^{+} \mathrm{m} / z$ calculated for $\mathrm{C}_{27} \mathrm{H}_{38} \mathrm{NO}_{13} \mathrm{~S}$ : 616.2058, found 616.2061.

$\beta$-D-Galactopyranose, $\quad 2,3,4,6$-tetraacetate $\quad 1-(2,6$ dimethoxybenzoate) [8a]

Compound 8a was prepared by using the general procedure A (reaction time: $15 \mathrm{~min}$ ), with commercial 3,5-dichlorobenzoic acid $\mathbf{5 c}$ (36.7 mg, $0.19 \mathrm{mmol}, 1.0$ equiv.) and $\mathbf{1 b}^{\mathrm{j}}$ (104.9 mg, 0.29 mmol, 1.5 equiv.), which afforded the product as a white solid $(70.7 \mathrm{mg}, 71 \%$ yield after column chromatography EtOAc/Cyclohexane 3:7); $\quad$ TLC $\quad \mathbf{R}_{\mathbf{f}}=(30 \%$ EtOAc/Cyclohexane) $=0.91 ;[\boldsymbol{\alpha}]_{\mathbf{D}}{ }^{\mathbf{2 5}}=-29.00$ (c 0,0031 $\mathrm{CH}_{2} \mathrm{Cl}_{2}$ ); IR (neat, $\mathbf{c m}^{-1}$ ) 2844, 1746, 1598, 1478,1369, 1232, 1113, 1053; ${ }^{\mathbf{1}} \mathbf{H}$ NMR (300 MHz, $\left.\mathbf{C D C l}_{3}\right) \boldsymbol{\delta}(\mathbf{p p m}) 7.22(\mathrm{~d}, J$ $=8.0 \mathrm{~Hz}, 1 \mathrm{H}), 6.48(\mathrm{~d}, J=8.5 \mathrm{~Hz}, 2 \mathrm{H}), 6.00(\mathrm{~d}, J=8.3 \mathrm{~Hz}$, $1 \mathrm{H}), 5.41-5.30(\mathrm{~m}, 2 \mathrm{H}), 5.08(\mathrm{dd}, J=10.4,3.4 \mathrm{~Hz}, 1 \mathrm{H}), 4.12$ $(\mathrm{d}, J=5.9 \mathrm{~Hz}, 1 \mathrm{H}), 4.06(\mathrm{dd}, J=7.4,2.0 \mathrm{~Hz}, 2 \mathrm{H}), 3.74(\mathrm{~s}$, 6H), $2.07(\mathrm{~s}, 3 \mathrm{H}), 2.01(\mathrm{~s}, 3 \mathrm{H}), 1.98(\mathrm{~d}, J=3.9 \mathrm{~Hz}, 3 \mathrm{H}), 1.94$ (s, 3H); ${ }^{13} \mathbf{C}\{1 \mathrm{H}\} \quad$ NMR (75 $\left.\mathbf{M H z}, \mathbf{C D C l}_{3}\right) \quad \delta \quad(\mathbf{p p m})$ $170.30\left(\mathrm{C}_{\mathrm{q}}\right), 170.16\left(\mathrm{C}_{\mathrm{q}}\right), 170.01\left(\mathrm{C}_{\mathrm{q}}\right), 169.20\left(\mathrm{C}_{\mathrm{q}}\right), 164.29\left(\mathrm{C}_{\mathrm{q}}\right)$, 157.67(2 x C $\left.)_{\mathrm{q}}\right), 131.82(\mathrm{CH}), 103.96(2 \times \mathrm{CH}), 92.46(\mathrm{CH})$, 71.85(CH), 71.14(CH), 68.07(CH), 66.79(CH), 60.90( $\left.\mathrm{CH}_{2}\right)$, 56.01(2 x CH 3$), 29.70\left(\mathrm{CH}_{3}\right), 20.63\left(3 \times \mathrm{CH}_{3}\right), 20.56\left(\mathrm{CH}_{3}\right)$; HRMS (ESI) $(\mathbf{M}+\mathbf{N a})^{+} \mathrm{m} / z$ calculated for $\mathrm{C}_{23} \mathrm{H}_{28} \mathrm{O}_{13} \mathrm{Na}$ : 535.1428, found 535.1428.

( $\beta$-D-Galactopyranose, 2,3,4,6-tetraacetate $\quad 1-(3,5-$ dichlorobenzoate $)[\mathbf{8 b}]$
Compound $\mathbf{8 b}$ was prepared by using the general procedure (reaction time: 1h), with commercial 3,5-dichlorobenzoic acid $\mathbf{5 m}$ (36.7 mg, 0.19 mmol, 1.0 equiv.) and $\mathbf{1 b}$ (104.9 mg, 0.29 mmol, 1.5 equiv.), which afforded the product as a white solid (70.7 mg, $71 \%$ yield after column chromatography EtOAc/Cyclohexane 3:7); TLC $\quad \mathbf{R}_{\mathbf{f}}=0.58$ (EtOAc/Cyclohexane, $\left.1: 1, \mathrm{SiO}_{2}\right)$; $\mathbf{m p}: 67.6-69.5{ }^{\circ} \mathrm{C} ;[\boldsymbol{\alpha}]_{\mathbf{D}}{ }^{20}$ $=-10.4\left(\mathrm{c}, 1.00\right.$ in $\left.\mathrm{CHCl}_{3}\right)$; IR (neat, $\left.\mathbf{~ c m}^{-1}\right)$ 3087, 1739, 1572, 1368, 1208, 1081, 1030; ${ }^{\mathbf{1}} \mathbf{H}$ NMR (300 $\left.\mathbf{M H z}, \mathbf{C D C l}_{3}\right) \delta$ (ppm) 7.92 (s, 2H), 7.58 (s, 1H), 5.89 (d, J = 8.3 Hz, 1H), 5.57 $-5.37(\mathrm{~m}, 2 \mathrm{H}), 5.16(\mathrm{dd}, J=10.4,3.5 \mathrm{~Hz}, 1 \mathrm{H}), 4.23-3.99$ (m, 3H), 2.19 (s, 3H), $2.04(\mathrm{~s}, 3 \mathrm{H}), 2.02(\mathrm{~s}, 3 \mathrm{H}), 2.00(\mathrm{~s}, 3 \mathrm{H})$; ${ }^{13} \mathbf{C}\{1 \mathrm{H}\}$ NMR (75 MHz, $\left.\mathbf{C D C l}_{3}\right) \boldsymbol{\delta}(\mathbf{p p m}) 170.5\left(\mathrm{C}_{\mathrm{q}}\right), 170.2$ $\left(\mathrm{C}_{\mathrm{q}}\right), 170.1\left(\mathrm{C}_{\mathrm{q}}\right), 169.5\left(\mathrm{C}_{\mathrm{q}}\right), 162.5\left(\mathrm{C}_{\mathrm{q}}\right), 135.7\left(2 \times \mathrm{C}_{\mathrm{q}}\right), 133.9$ $(\mathrm{CH}), 131.4\left(\mathrm{C}_{\mathrm{q}}\right), 128.6(2 \times \mathrm{CH}), 93.4(\mathrm{CH}), 72.0(\mathrm{CH}), 70.7$ $(\mathrm{CH}), 67.8(\mathrm{CH}), 66.9(\mathrm{CH}), 61.1\left(\mathrm{CH}_{2}\right), 20.8\left(3 \times \mathrm{CH}_{3}\right), 20.7$ $\left(\mathrm{CH}_{3}\right)$; HRMS (ESI) $(\mathbf{M}+\mathbf{N a})^{+} \mathrm{m} / \mathrm{z}$ calculated for $\mathrm{C}_{21} \mathrm{H}_{22} \mathrm{Cl}_{2} \mathrm{O}_{11} \mathrm{Na}$ 543.0437, found 543.0433.

$\beta$-D-Mannopyranose, $\quad$ 2,3,4,6-tetraacetate $\quad 1-(3,5$ dichlorobenzoate) $[\mathbf{8 c}]$

Compound $8 \mathbf{c}$ was prepared by using the general procedure (reaction time: 1h), with commercial 3,5-dichlorobenzoic acid 5m (36.7 mg, 0.19 mmol, 1.0 equiv.) and 1c (104.9 mg, 0.29 mmol, 1.5 equiv.), which afforded the product as a white solid $(42.8 \mathrm{mg}, 43 \%$ yield after column chromatography EtOAc/Cyclohexane 3:7); TLC $\quad \mathbf{R}_{\mathbf{f}}=0.58$ (EtOAc/Cyclohexane, $\left.1: 1, \mathrm{SiO}_{2}\right)$; $\mathbf{~ m p : ~} 80.0-81.5{ }^{\circ} \mathrm{C} ;[\boldsymbol{\alpha}]_{\mathbf{D}}{ }^{20}$ $=+26.3\left(\mathrm{c}, 1.00\right.$ in $\left.\mathrm{CHCl}_{3}\right) ;$ IR (neat, $\left.\mathbf{~ c m}^{-1}\right) 3087,2959,1750$, 1572, 1369, 1271, 1095, 1051; ${ }^{1} \mathbf{H}$ NMR (300 MHz, $\left.\mathbf{C D C l}_{3}\right) \boldsymbol{\delta}$ (ppm) $7.91(\mathrm{~d}, J=1.7 \mathrm{~Hz}, 2 \mathrm{H}), 7.67-7.56(\mathrm{~m}, 1 \mathrm{H}), 6.31(\mathrm{~s}$, $1 \mathrm{H}), 5.49-5.35(\mathrm{~m}, 3 \mathrm{H}), 4.32(\mathrm{dd}, J=12.6,5.3 \mathrm{~Hz}, 1 \mathrm{H}), 4.12$ (dd, $J=9.3,3.4 \mathrm{~Hz}, 2 \mathrm{H}), 2.21(\mathrm{~s}, 3 \mathrm{H}), 2.09(\mathrm{~s}, 6 \mathrm{H}), 2.04(\mathrm{~s}$, $3 \mathrm{H}) ;{ }^{13} \mathbf{C}\{1 \mathrm{H}\}$ NMR (75 MHz, $\left.\mathbf{C D C l}_{3}\right) \delta$ (ppm) $170.7\left(\mathrm{C}_{\mathrm{q}}\right)$, $170.1\left(\mathrm{C}_{\mathrm{q}}\right), 169.8\left(\mathrm{C}_{\mathrm{q}}\right), 169.6\left(\mathrm{C}_{\mathrm{q}}\right), 161.8\left(\mathrm{C}_{\mathrm{q}}\right), 135.9\left(2 \times \mathrm{C}_{\mathrm{q}}\right)$, 134.0 $(\mathrm{CH}), 131.6\left(\mathrm{C}_{\mathrm{q}}\right), 128.4(2 \times \mathrm{CH}), 92.2(\mathrm{CH}), 71.4(\mathrm{CH})$, $68.9(\mathrm{CH}), 68.3(\mathrm{CH}), 65.4(\mathrm{CH}), 62.1\left(\mathrm{CH}_{2}\right), 20.9\left(\mathrm{CH}_{3}\right), 20.8$ $\left(3 \times \mathrm{CH}_{3}\right)$; HRMS (ESI) $(\mathbf{M}+\mathbf{N a})^{+} \mathrm{m} / \mathrm{z}$ calculated for $\mathrm{C}_{21} \mathrm{H}_{22} \mathrm{Cl}_{2} \mathrm{O}_{11} \mathrm{Na}$ 543.0437, found 543.0444.

$\beta$-D-Fucopyranose, $\quad$ 2,3,4,6-tetraacetate $\quad$ 1-(2,6-dimethoxy benzoate) [8d]

Compound 8d was prepared by using the general procedure (reaction time: $15 \mathrm{~min}$ ), with commercial 2,6dimethoxybenzoic acid 5c (27.75 mg, $0.152 \mathrm{mmol}, 1.0$ equiv.) and $1 \mathbf{d}$ (70.0 $\mathrm{mg}, 0.228 \mathrm{mmol}, 1.5$ equiv.), which afforded the product as a white oil $(50 \mathrm{mg}, 72 \%$ yield after column chromatography $(1 \% \mathrm{MeOH} / \mathrm{DCM}) ; \quad \mathbf{T L C} \quad \mathbf{R}_{\mathbf{f}}=(50 \%$ EtOAc/Cyclohexane) $=0.63 ;[\boldsymbol{\alpha}]_{\mathbf{D}}{ }^{\mathbf{2 5}}=-16.68$ (c 0.0016 $\mathrm{CH}_{2} \mathrm{Cl}_{2}$ ); IR (neat, $\mathbf{c m}^{-\mathbf{1}}$ ) 1575, $1598,1257,1056,726 ;{ }^{\mathbf{1}} \mathbf{H}$ NMR (300 MHz, CDCl $\mathbf{M}_{3}$ ) (ppm) $7.29(\mathrm{t}, J=8.4 \mathrm{~Hz}, 1 \mathrm{H})$, $6.54(\mathrm{~d}, J=8.5 \mathrm{~Hz}, 2 \mathrm{H}), 6.05(\mathrm{~d}, J=8.3 \mathrm{~Hz}, 1 \mathrm{H}), 5.45-5.33$ $(\mathrm{m}, 1 \mathrm{H}), 5.29(\mathrm{~d}, J=3.3 \mathrm{~Hz}, 1 \mathrm{H}), 5.15(\mathrm{dd}, 1 \mathrm{H}), 4.16-4.07$ $(\mathrm{m}, 1 \mathrm{H}), 3.80(\mathrm{~s}, 6 \mathrm{H}), 2.16(\mathrm{~s}, 3 \mathrm{H}), 2.08(\mathrm{~s}, 3 \mathrm{H}), 2.00(\mathrm{~s}, 3 \mathrm{H})$, $1.31-1.24(\mathrm{~m}, J=6.5,1.9 \mathrm{~Hz}, 3 \mathrm{H}) ;{ }^{13} \mathbf{C}\{\mathbf{1 H}\}$ NMR $(75$ MHz, $\left.\left.\mathrm{CDCl}_{3}\right) \delta(\mathrm{ppm}){ }^{13} \mathrm{C} \mathrm{NMR} \mathrm{(75} \mathrm{MHz,} \mathrm{CDCl}_{3}\right) \delta(\mathrm{ppm})$ $170.55\left(\mathrm{C}_{\mathrm{q}}\right), 170.04\left(\mathrm{C}_{\mathrm{q}}\right), 169.30\left(\mathrm{C}_{\mathrm{q}}\right), 164.43\left(\mathrm{C}_{\mathrm{q}}\right), 157.69(2 \mathrm{x}$ $\left.\mathrm{C}_{\mathrm{q}}\right), 131.67(\mathrm{CH}), 111.59(2 \times \mathrm{CH}), 103.95(\mathrm{CH}), 92.50(\mathrm{CH})$, 71.59(CH), 70.50(CH), 70.10(CH), 68.24(CH), 55.99 $(2 \mathrm{x}$ $\left.\mathrm{CH}_{3}\right), \quad 20.66\left(\mathrm{CH}_{3}\right), \quad 20.61\left(\mathrm{CH}_{3}\right), \quad 20.57\left(\mathrm{CH}_{3}\right), \quad 15.98\left(\mathrm{CH}_{3}\right)$; HRMS (ESI) $(\mathbf{M}+\mathbf{H})^{+} \mathrm{m} / z$ calculated for $\mathrm{C}_{21} \mathrm{H}_{27} \mathrm{O}_{11}$ : 455.15479 , found 455.1542 . 
$\beta$-D-Glucopyranose, dichlorobenzoate) $[\mathbf{8 e}]$

Compound 8e was prepared by using the general procedure (reaction time: 1h), with commercial 3,5-dichlorobenzoic acid $5 \mathrm{~m}(36.7 \mathrm{mg}, 0.19 \mathrm{mmol}, 1.0$ equiv.) and $1 \mathrm{e}(176.3 \mathrm{mg}, 0.29$ mmol, 1.5 equiv.), which afforded the product as a white solid (75.1 mg, 51\% yield after column chromatography EtOAc/Cyclohexane 3:7); $\quad$ TLC $\quad \mathbf{R}_{\mathbf{f}}=0.84$ (EtOAc/Cyclohexane, 1:1, $\mathrm{SiO}_{2}$ ); mp: $100.9-101.5{ }^{\circ} \mathrm{C}$; $[\boldsymbol{\alpha}]_{\mathbf{D}}{ }^{\mathbf{2 0}}=+8.9\left(\mathrm{c}, 1.00\right.$ in $\left.\mathrm{CHCl}_{3}\right) ;$ IR (neat, $\mathbf{c m}^{-\mathbf{1}}$ ) 3067, 2969, 1722, 1602, 1452, 1316, 1091, 1086, 1026; ${ }^{1}$ H NMR (300 MHz, $\left.\mathbf{C D C l}_{3}\right) \boldsymbol{\delta}$ (ppm) $8.05(\mathrm{~d}, J=7.3 \mathrm{~Hz}, 2 \mathrm{H}), 7.94(\mathrm{dd}, J=$ 7.5, 4.1 Hz, 4H), 7.90 (s, 1H), 7.89 (s, 2H), 7.87 (s, 1H), 7.56 $(\mathrm{d}, J=7.3 \mathrm{~Hz}, 1 \mathrm{H}), 7.54-7.51(\mathrm{~m}, 2 \mathrm{H}), 7.51-7.47(\mathrm{~m}, 1 \mathrm{H})$, $7.44(\mathrm{dd}, J=7.3,2.8 \mathrm{~Hz}, 2 \mathrm{H}), 7.37$ (d, $J=7.3 \mathrm{~Hz}, 3 \mathrm{H}), 7.32$ $(\mathrm{d}, J=7.8 \mathrm{~Hz}, 3 \mathrm{H}), 7.28(\mathrm{~s}, 1 \mathrm{H}), 6.27(\mathrm{~d}, J=7.9 \mathrm{~Hz}, 1 \mathrm{H}), 6.06$ (t, $J=9.4 \mathrm{~Hz}, 1 \mathrm{H}), 5.84(\mathrm{dd}, J=13.5,5.8 \mathrm{~Hz}, 2 \mathrm{H}), 4.69$ (dd, $J$ $=12.3,2.7 \mathrm{~Hz}, 1 \mathrm{H}), 4.52(\mathrm{dd}, J=12.3,4.7 \mathrm{~Hz}, 1 \mathrm{H}), 4.47-$ $4.36(\mathrm{~m}, 1 \mathrm{H}) ;{ }^{13} \mathbf{C}\{\mathbf{1 H}\}$ NMR (75 $\left.\mathbf{M H z}, \mathbf{C D C l}_{3}\right) \boldsymbol{\delta}(\mathbf{p p m})$ $166.2\left(\mathrm{C}_{\mathrm{q}}\right), 165.8\left(\mathrm{C}_{\mathrm{q}}\right), 165.2\left(2 \times \mathrm{C}_{\mathrm{q}}\right), 162.6\left(\mathrm{C}_{\mathrm{q}}\right), 135.6(3 \mathrm{x}$ $\left.\mathrm{C}_{\mathrm{q}}\right), 133.8(2 \times \mathrm{CH}), 133.7(2 \times \mathrm{CH}), 133.5(\mathrm{CH}), 133.3(\mathrm{CH})$, $131.5\left(\mathrm{C}_{\mathrm{q}}\right), 130.0(4 \times \mathrm{CH}), 129.9(4 \times \mathrm{CH}), 129.6\left(\mathrm{C}_{\mathrm{q}}\right), 128.7$ $\left(2 \times \mathrm{C}_{\mathrm{q}}\right), 128.6(5 \times \mathrm{CH}), 128.5(4 \times \mathrm{CH}), 93.4(\mathrm{CH}), 73.5$ (CH), $72.7(\mathrm{CH}), 71.0(\mathrm{CH}), 69.0(\mathrm{CH}), 62.7\left(\mathrm{CH}_{2}\right)$; HRMS (ESI) $(\mathbf{M}+\mathbf{N a})^{+} \mathrm{m} / z$ calculated for $\mathrm{C}_{41} \mathrm{H}_{30} \mathrm{Cl}_{2} \mathrm{O}_{11} \mathrm{Na} 791.1063$, found 791.1062.

$\alpha$-D-Glucopyranose, 2,3,4,6-tetra-O-methyl $\quad 1-(2,6-$ dimethoxybenzoate) $[\mathbf{\alpha}-\mathbf{8 f}]$

Compounds $\boldsymbol{\alpha}-\mathbf{8 f}$ and $\boldsymbol{\beta}-\mathbf{8 f}$ were prepared by using the procedure (A) (reaction time: 15 min) with commercial 2,6-dimethoxybenzoic acid 5c (91.46 mg, $0.502 \mathrm{mmol}, 1.0$ equiv.) and 1f (190.0 $\mathrm{mg}, 0.753 \mathrm{mmol}, 1.5$ equiv.), which afforded the product as a yellow oil in a mixture of $\alpha: \beta(1: 1$ ratio) (184 mg, 91\%). After purification through column chromatography EtOAc/Cyclohexane 5:5), both anomers were isolated and characterized:

Anomer $\boldsymbol{\alpha}-\mathbf{8 f}: \quad 45 \%$ yield, $\quad$ TLC $\quad \mathbf{R}_{\mathbf{f}}=(50 \%$ EtOAc/Cyclohexane $)=0.4 ;[\boldsymbol{\alpha}]_{\mathbf{D}}{ }^{\mathbf{2 5}}=123.07$ (c 0.0016 $\mathrm{CH}_{2} \mathrm{Cl}_{2}$ ); IR (neat, $\mathbf{c m}^{-1}$ ) 1752, 1597, 1476, 1257, 1091, 730; ${ }^{1} \mathbf{H}$ NMR (300 MHz, $\left.\mathbf{C D C l}_{\mathbf{3}}\right) \boldsymbol{\delta}(\mathbf{p p m}) 7.28(\mathrm{t}, J=8.4 \mathrm{~Hz}$, $1 \mathrm{H}), 6.54(\mathrm{~d}, J=8.4 \mathrm{~Hz}, 2 \mathrm{H}), 5.72(\mathrm{~d}, J=7.8 \mathrm{~Hz}, 1 \mathrm{H}), 3.77$ $(\mathrm{d}, J=15.4 \mathrm{~Hz}, 6 \mathrm{H}), 3.68-3.67(\mathrm{~m}, J=1.9 \mathrm{~Hz}, 1 \mathrm{H}), 3.65(\mathrm{~s}$, $3 \mathrm{H}), 3.63(\mathrm{~d}, J=3.8 \mathrm{~Hz}, 1 \mathrm{H}), 3.57(\mathrm{~s}, 3 \mathrm{H}), 3.55(\mathrm{~s}, 3 \mathrm{H}), 3.50-$ $3.45(\mathrm{~m}, 1 \mathrm{H}), 3.41(\mathrm{~s}, 3 \mathrm{H}), 3.29(\mathrm{dd}, J=10.5,6.1 \mathrm{~Hz}, 2 \mathrm{H})$, $3.25-3.18(\mathrm{~m}, 1 \mathrm{H}) ;{ }^{13} \mathbf{C}\{\mathbf{1 H}\} \mathbf{N M R}\left(\mathbf{7 5} \mathbf{M H z}, \mathbf{C D C l}_{3}\right) \delta$ (ppm) $164.90\left(\mathrm{C}_{\mathrm{q}}\right), \quad 157.40\left(\mathrm{C}_{\mathrm{q}}\right), \quad 131.28(\mathrm{CH}), \quad 112.52\left(\mathrm{C}_{\mathrm{q}}\right)$,

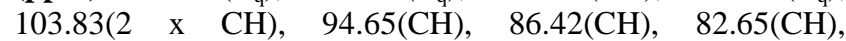
78.78( $\mathrm{CH}), 75.73(\mathrm{CH}), 70.75\left(\mathrm{C}_{\mathrm{q}}\right), 60.81\left(\mathrm{CH}_{3}\right), 60.29\left(\mathrm{CH}_{3}\right)$, 60.15 $\left(\mathrm{CH}_{3}\right)$, 59.30 $\left(\mathrm{CH}_{3}\right)$, 55.85(2 x CH), 29.63( $\left(\mathrm{CH}_{2}\right)$; HRMS (ESI) $(\mathbf{M}+\mathbf{H})^{+} \mathrm{m} / z$ calculated for $\mathrm{C}_{19} \mathrm{H}_{29} \mathrm{O}_{9}: 401.1806$, found 401.1766.

\section{$\beta$-D-Glucopyranose, $\quad$ 2,3,4,6-tetra-O-methyl $\quad 1-(2,6-$} dimethoxybenzoate) $[\boldsymbol{\beta}-\mathbf{8 f}]$

Anomer $\boldsymbol{\beta}-\mathbf{8 f}$ : $45 \%$ yield, TLC $\quad \mathbf{R}_{\mathbf{f}}=(50 \%$ EtOAc/Cyclohexane $)=0.52 ;[\boldsymbol{\alpha}]_{\mathbf{D}}{ }^{25}=235.72(\mathrm{c} 0.0016$ $\mathrm{CH}_{2} \mathrm{Cl}_{2}$ ); IR (neat, $\mathbf{c m}^{-1}$ ) 1745, 1596, 1476, 1257, 1097, 990; ${ }^{1} \mathbf{H}$ NMR (300 MHz, $\mathbf{C D C l}_{3}$ ) $\boldsymbol{\delta}$ (ppm) 7.28 (s, 1H), 6.54 (d, $J$ $=8.4 \mathrm{~Hz}, 2 \mathrm{H}), 6.49(\mathrm{~d}, J=3.5 \mathrm{~Hz}, 1 \mathrm{H}), 3.95-3.86(\mathrm{~m}, J=$ 12.9, $5.4 \mathrm{~Hz}, 1 \mathrm{H}), 3.80(\mathrm{~s}, 6 \mathrm{H}), 3.62(\mathrm{~s}, 2 \mathrm{H}), 3.61(\mathrm{~s}, 3 \mathrm{H}), 3.55$ (s, 3H), 3.49 (s, 3H), $3.40(\mathrm{~s}, 3 \mathrm{H}), 3.39(\mathrm{~d}, J=3.5 \mathrm{~Hz}, 1 \mathrm{H})$, $3.36(\mathrm{~d}, J=3.6 \mathrm{~Hz}, 1 \mathrm{H}), 3.32-3.26(\mathrm{~m}, J=9.5 \mathrm{~Hz}, 1 \mathrm{H}$; ${ }^{13} \mathbf{C}\{1 \mathrm{H}\}$ NMR (75 MHz, $\left.\mathbf{C D C l}_{3}\right) \delta$ (ppm) 164.78(Cq),
157.81(Cq), $131.38(\mathrm{CH}), \quad 112.44(\mathrm{Cq}), \quad 104.02(2 \quad x \quad \mathrm{CH})$, 89.96(CH), 83.05 $(\mathrm{CH}), 80.52,(\mathrm{CH}) 78.82(\mathrm{CH}), 72.41(\mathrm{CH})$, 70.67(Cq), 60.73( $\left(\mathrm{CH}_{3}\right), 60.45\left(\mathrm{CH}_{3}\right), 59.14\left(\mathrm{CH}_{3}\right), 58.24\left(\mathrm{CH}_{3}\right)$, 55.91(2 x CH), 29.65( $\left.\mathrm{CH}_{2}\right)$; HRMS (ESI) $(\mathbf{M}+\mathbf{H})^{+} \mathrm{m} / \mathrm{z}$ calculated for $\mathrm{C}_{19} \mathrm{H}_{29} \mathrm{O}_{9}$ : 401.1806, found 401.1766.

D-Glucopyranose, 2,4,6-tri-O-[tert-butyldimethylsilyl] 1 benzoate [8g]

Compound $\mathbf{8 g}$ was prepared by using the general procedure A (reaction time: $15 \mathrm{~min}$ ), with commercial benzoic acid $\mathbf{5 a}(1.0$ equiv.) and $\mathbf{1 g}$ (1.2 equiv.), which afforded the product as a colourless oil in a mixture of two isomers $2: 1$ ratio $(36 \mathrm{mg}$, $70 \%$ yield after column chromatography EtOAc/Cyclohexane 3:7); $\mathbf{T L C} \mathbf{R}_{\mathbf{f}}=0.33\left(\right.$ EtOAc/Cyclohexane, 95:5, $\left.\mathrm{SiO}_{2}\right) ;{ }^{1} \mathbf{H}$ NMR (300 MHz, CDCl $\mathbf{~}_{3} \boldsymbol{\delta}$ (ppm) 8.08 (m, 2H, H-2, H-6), 7.59 (m, 1H, H-4), 7.47 (dt, J = 15.1, 7.6 Hz, 2H, H-3, H-5), $6.33\left(\mathrm{~d}, \mathrm{~J}=3.4 \mathrm{~Hz}, 0.3 \mathrm{H}, \mathrm{H}-1^{\prime}\right), 6.09-5.99\left(\mathrm{~m}, 0.7 \mathrm{H}, \mathrm{H}-1^{\prime}\right)$, $4.08-3.93(\mathrm{~m}, 2 \mathrm{H}),, 3.85(\mathrm{~d}, \mathrm{~J}=2.7 \mathrm{~Hz}, 2 \mathrm{H}),, 3.81-3.58$ $(\mathrm{m}, 2 \mathrm{H}),, 3.24(\mathrm{~d}, \mathrm{~J}=4.1 \mathrm{~Hz}, 0.7 \mathrm{H}, \mathrm{OH}), 2.82(\mathrm{~d}, \mathrm{~J}=2.7 \mathrm{~Hz}$, $0.3 \mathrm{H}, \mathrm{OH}), 0.98$ (s, 3H, $\left.\mathrm{CH}_{3}\right), 0.89$ (dd, J = 4.4, $2.3 \mathrm{~Hz}, 21 \mathrm{H}$, $\left.7 \mathrm{XCH}_{3}\right), 0.82\left(\mathrm{~s}, 3 \mathrm{H}, \mathrm{CH}_{3}\right), 0.21\left(\mathrm{~d}, \mathrm{~J}=15.0 \mathrm{~Hz}, 3 \mathrm{H}, \mathrm{CH}_{3}\right)$, $0.18-0.14\left(\mathrm{~m}, 6 \mathrm{H}, 2 \mathrm{XCH}_{3}\right), 0.12\left(\mathrm{~d}, \mathrm{~J}=2.0 \mathrm{~Hz}, 3 \mathrm{H}, \mathrm{CH}_{3}\right)$, $0.09\left(\mathrm{~d}, \mathrm{~J}=4.5 \mathrm{~Hz}, 3 \mathrm{H}, \mathrm{CH}_{3}\right), 0.05\left(\mathrm{~s}, 3 \mathrm{H}, \mathrm{CH}_{3}\right) .{ }^{13} \mathbf{C}\{\mathbf{1 H}\}$ NMR (75 MHz, CDCl ) $^{\delta}$ (ppm) $164.23(\mathrm{C}=\mathrm{O}), 132.25(\mathrm{Ph})$, $129.02(\mathrm{Ph}), 128.83(\mathrm{C} 1), 128.76(\mathrm{Ph}), 127.46(\mathrm{Ph})$, 127.25(Ph), 93.57 (C1'), 91.77 (C1'), 74.85, 74.66, 74.00, 71.98, 71.65, 71.50, 70.58, 63.35 (C6'), 63.19 (C6'), 25.18 $\left(\mathrm{SiC}\left(\mathrm{CH}_{3}\right)_{3}\right), 24.97\left(\mathrm{SiC}\left(\mathrm{CH}_{3}\right)_{3}\right), 24.77\left(\mathrm{SiC}\left(\mathrm{CH}_{3}\right)_{3}\right), \quad 17.40$ $\left(\mathrm{SiC}\left(\mathrm{CH}_{3}\right)_{3}\right), 17.23\left(\mathrm{SiC}\left(\mathrm{CH}_{3}\right)_{3}\right), 16.93\left(\mathrm{SiC}\left(\mathrm{CH}_{3}\right)_{3}\right),-5.03$ $\left(\mathrm{SiCH}_{3}\right),-5.08\left(\mathrm{SiCH}_{3}\right),-5.18\left(\mathrm{SiCH}_{3}\right),-5.30\left(\mathrm{SiCH}_{3}\right),-5.42$ $\left(\mathrm{SiCH}_{3}\right),-6.46\left(\mathrm{SiCH}_{3}\right)$. HRMS (ESI) $(\mathbf{M}+\mathbf{N a})^{+} \mathrm{m} / z$ calculated for $\mathrm{C}_{31} \mathrm{H}_{62} \mathrm{NO}_{7} \mathrm{Si}_{3}{ }^{+}$644.3832, found 644.3834.

$\beta$-D-Cellobiose, $\quad 2,2^{\prime}, 3,3^{\prime}, 4^{\prime}, 6,6^{\prime}$-heptaacetate $\quad 1-(2,6$ dimethoxybenzoate) [8h]

Compound $\mathbf{8 h}$ was prepared by using the general procedure A (reaction time: $15 \mathrm{~min}$ ), with commercial 2,6dimethoxybenzoic acid 5c ( (18.58 $\mathrm{mg}, 0.102 \mathrm{mmol}, 1.0$ equiv.) and $\mathbf{1 h}$ (99.85 $\mathrm{mg}, 0.153 \mathrm{mmol}, 1.5$ equiv.), which afforded the product as a yellow oil $(43.3 \mathrm{mg}, 53 \%$ yield after column chromatography $2 \% \mathrm{MeOH} / \mathrm{DCM}) ;$ TLC $\mathbf{R}_{\mathbf{f}}=(50 \%$ $\mathrm{MeOH} / \mathrm{DCM})=0.29 ;[\boldsymbol{\alpha}]_{\mathbf{D}}{ }^{25}=-43.75\left(\mathrm{c} 0,0032 \mathrm{CH}_{2} \mathrm{Cl}_{2}\right) ; \mathbf{I R}$ (neat, $\mathbf{c m}^{-1}$ ) 3400, 1759, 1738, 1364, 1212, 1114, 1042; ${ }^{\mathbf{1}} \mathbf{H}$ NMR (300 MHz, CDCl $\left.\mathbf{~}_{3}\right) \boldsymbol{\delta}$ (ppm) $7.22(\mathrm{~d}, J=6.5 \mathrm{~Hz}, 1 \mathrm{H})$, $6.47(\mathrm{~d}, J=8.4 \mathrm{~Hz}, 2 \mathrm{H}), 5.96(\mathrm{~d}, J=8.1 \mathrm{~Hz}, 1 \mathrm{H}), 5.26-5.18$ $(\mathrm{m}, J=8.9 \mathrm{~Hz}, 1 \mathrm{H}), 5.14-5.03(\mathrm{~m}, 2 \mathrm{H}), 4.99(\mathrm{~d}, J=9.6 \mathrm{~Hz}$, $1 \mathrm{H}), 4.87$ (t, $J=8.4 \mathrm{~Hz}, 1 \mathrm{H}), 4.51-4.43(\mathrm{~m}, 2 \mathrm{H}), 4.30$ (dd, $J$ $=12.4,4.5 \mathrm{~Hz}, 1 \mathrm{H}), 4.13(\mathrm{dd}, J=12.2,4.5 \mathrm{~Hz}, 1 \mathrm{H}), 4.03-$ $3.97(\mathrm{~m}, J=12.6 \mathrm{~Hz}, 1 \mathrm{H}), 3.86-3.80(\mathrm{~m}, J=6.7 \mathrm{~Hz}, 1 \mathrm{H})$, $3.79-3.75(\mathrm{~m}, 1 \mathrm{H}), 3.73(\mathrm{~s}, 6 \mathrm{H}), 3.64-3.57(\mathrm{~m}, J=9.8 \mathrm{~Hz}$, 1H), 2.06 (s, 3H), 2.04 (s, 3H), 1.98 (s, 6H), 1.97 (s, 3H), 1.95 $(\mathrm{s}, 3 \mathrm{H}), 1.93(\mathrm{~s}, 3 \mathrm{H}) ;{ }^{13} \mathbf{C}\{\mathbf{1 H}\} \mathbf{N M R}\left(\mathbf{7 5} \mathbf{M H z}, \mathbf{C D C l}_{3}\right) \delta$ (ppm) $169.52\left(\mathrm{C}_{\mathrm{q}}\right), \quad 169.27\left(\mathrm{C}_{\mathrm{q}}\right), \quad 169.22\left(\mathrm{C}_{\mathrm{q}}\right), \quad 168.71\left(\mathrm{C}_{\mathrm{q}}\right)$, $168.46\left(\mathrm{C}_{\mathrm{q}}\right), 168.29\left(\mathrm{C}_{\mathrm{q}}\right), 168.01\left(\mathrm{C}_{\mathrm{q}}\right), 163.08\left(\mathrm{C}_{\mathrm{q}}\right), 156.73(2 \mathrm{x}$ $\left.\mathrm{C}_{\mathrm{q}}\right), 130.85(\mathrm{CH}), 110.30\left(\mathrm{C}_{\mathrm{q}}\right), 102.97(2 \times \mathrm{CH}), 99.61(\mathrm{CH})$, 90.96(CH), 74.93(CH), 72.74(CH), 71.92(CH), 71.61(CH), 70.99 $(\mathrm{CH}), 70.60(\mathrm{CH}), 69.81(\mathrm{CH}), 66.85(\mathrm{CH}), 60.69\left(\mathrm{CH}_{2}\right)$, 60.59 $\left(\mathrm{CH}_{2}\right), 54.99\left(2 \times \mathrm{CH}_{3}\right), 19.81\left(\mathrm{CH}_{3}\right), 19.63\left(\mathrm{CH}_{3}\right), 19.53(5$ x $\left.\mathrm{CH}_{3}\right)$; HRMS (ESI) $(\mathbf{M}+\mathbf{N a})^{+} \mathrm{m} / \mathrm{z}$ calculated for $\mathrm{C}_{35} \mathrm{H}_{44} \mathrm{O}_{21} \mathrm{Na}$ : 823.2273, found 823.2275. 
$\beta$-D-Cellobiose, $\quad 2,2^{\prime}, 3,3^{\prime}, 4^{\prime}, 6,6^{\prime}$-heptaacetate $\quad 1-(3,5$ dichlorobenzoate) [8i]

Compound $\mathbf{8 i}$ was prepared by using the general procedure $\mathrm{B}$ (reaction time: 1h), with commercial 3,5-dichlorobenzoic acid $\mathbf{5 m ~ ( 3 6 . 7 ~ m g , ~} 0.19 \mathrm{mmol}, 1.0$ equiv.) and $\mathbf{1 h}(187.8 \mathrm{mg}, 0.29$ mmol, 1.5 equiv.), which afforded the product as a white solid (69.1 mg, 45\% yield after column chromatography EtOAc/Cyclohexane 3:7); $\quad$ TLC $\quad \mathbf{R}_{\mathbf{f}}=0.41$ (EtOAc/Cyclohexane, 1:1, $\mathrm{SiO}_{2}$ ); mp: $162.2-164.0{ }^{\circ} \mathrm{C}$; $[\boldsymbol{\alpha}]_{\mathbf{D}}{ }^{\mathbf{2 0}}=-10.7\left(\mathrm{c}, 1.00\right.$ in $\left.\mathrm{CHCl}_{3}\right)$; IR (neat, $\mathbf{c m}^{-\mathbf{1}}$ ) 2949, 1757, 1745, 1368, 1230, 1215, 1168, 1037; ${ }^{\mathbf{1}} \mathbf{H}$ NMR (300 MHz, $\left.\mathbf{C D C l}_{3}\right) \delta(\mathbf{p p m}) 7.87(\mathrm{~d}, J=2.2 \mathrm{~Hz}, 2 \mathrm{H}), 7.58(\mathrm{t}, J=2.2 \mathrm{~Hz}$, $1 \mathrm{H}), 5.87(\mathrm{~d}, J=7.8 \mathrm{~Hz}, 1 \mathrm{H}), 5.34-5.21(\mathrm{~m}, 2 \mathrm{H}), 5.11(\mathrm{dt}, J$ $=18.9,9.7 \mathrm{~Hz}, 2 \mathrm{H}), 4.98-4.90(\mathrm{~m}, 1 \mathrm{H}), 4.56-4.46(\mathrm{~m}, 2 \mathrm{H})$, $4.37(\mathrm{dd}, J=12.5,4.5 \mathrm{~Hz}, 1 \mathrm{H}), 4.13(\mathrm{dd}, J=12.2,4.2 \mathrm{~Hz}$, $1 \mathrm{H}), 4.06(\mathrm{dd}, J=12.5,2.4 \mathrm{~Hz}, 1 \mathrm{H}), 3.93-3.79(\mathrm{~m}, 2 \mathrm{H}), 3.68$ (ddd, $J=9.7,4.6,2.3 \mathrm{~Hz}, 1 \mathrm{H}), 2.11(\mathrm{~s}, 3 \mathrm{H}), 2.10(\mathrm{~s}, 3 \mathrm{H}), 2.06$ (s, 3H), $2.04(\mathrm{~s}, 3 \mathrm{H}), 2.01(\mathrm{~s}, 3 \mathrm{H}), 2.00(\mathrm{~s}, 3 \mathrm{H}), 1.99(\mathrm{~s}, 3 \mathrm{H})$; ${ }^{13} \mathbf{C}\{1 \mathrm{H}\}$ NMR (75 MHz, $\left.\mathbf{C D C l}_{3}\right) \boldsymbol{\delta}$ (ppm) $170.6\left(\mathrm{C}_{\mathrm{q}}\right), 170.4$ $\left(2 \times \mathrm{C}_{\mathrm{q}}\right), 169.7\left(\mathrm{C}_{\mathrm{q}}\right), 169.6\left(\mathrm{C}_{\mathrm{q}}\right), 169.5\left(\mathrm{C}_{\mathrm{q}}\right), 169.2\left(\mathrm{C}_{\mathrm{q}}\right), 162.4$ $\left(\mathrm{C}_{\mathrm{q}}\right), 135.7\left(2 \times \mathrm{C}_{\mathrm{q}}\right), 133.9(\mathrm{CH}), 131.5\left(\mathrm{C}_{\mathrm{q}}\right), 128.6(2 \mathrm{x} \mathrm{CH})$, $100.9(\mathrm{CH}), 92.8(\mathrm{CH}), 76.0(\mathrm{CH}), 73.8(\mathrm{CH}), 73.0(\mathrm{CH}), 72.3$ $(\mathrm{CH}), 72.2(\mathrm{CH}), 71.7(\mathrm{CH}), 70.4(\mathrm{CH}), 67.9(\mathrm{CH}), 61.8$ $\left(\mathrm{CH}_{2}\right), 61.7\left(\mathrm{CH}_{2}\right), 21.0\left(\mathrm{CH}_{3}\right), 20.8\left(\mathrm{CH}_{3}\right), 20.7\left(5 \times \mathrm{CH}_{3}\right)$; HRMS (ESI) $(\mathbf{M}+\mathbf{N a})^{+} \mathrm{m} / z$, calculated for $\mathrm{C}_{33} \mathrm{H}_{38} \mathrm{Cl}_{2} \mathrm{O}_{19} \mathrm{Na}$ 831.1277, found 831.1299.

$\beta$-D-Glucopyranose, $O-2,3,4,6$-tetra-O-acetyl- $\alpha-D$ -

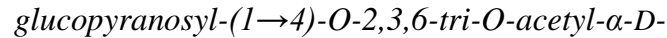

glucopyranosyl- $(1 \rightarrow 4)-, \quad$ 2,3,6-triacetate $\quad 1-(2,6$ dimethoxybenzoate) [8j]

Compound $8 \mathbf{j}$ was prepared by using the general procedure A (reaction time: $15 \mathrm{~min}$ ), with commercial 2,6dimethoxybenzoic acid 5c (10.2 $\mathrm{mg}, 0.056 \mathrm{mmol}, 1.0$ equiv.) and $1 \mathbf{1}^{[25]}$ (79.0 mg, $0.084 \mathrm{mmol}, 1.5$ equiv.), which afforded the product as a white solid $(25.6 \mathrm{mg}, 42 \%$ yield after column chromatography $(1 \% \mathrm{MeOH} / \mathrm{DCM}) ;$ TLC $\mathbf{R}_{\mathbf{f}}=\mathbf{R}_{\mathbf{f}}(50 \%$ EtOAc/Cyclohexane $)=0.17$; $\mathbf{m p :} 92-101{ }^{\circ} \mathrm{C} ;[\boldsymbol{\alpha}]_{\mathbf{D}}{ }^{25}=+28.88$ (c 0,0045 $\mathrm{CH}_{2} \mathrm{Cl}_{2}$ ); IR (neat, $\mathbf{c m}^{-1}$ ) 3260,2978, 1757, 1641, 1369, 1297, 1115, 1044; ${ }^{\mathbf{1}} \mathbf{H}$ NMR (300 $\left.\mathbf{~ M H z , ~} \mathbf{C D C l}_{3}\right) \boldsymbol{\delta}$ (ppm) $7.22(\mathrm{~d}, J=6.6 \mathrm{~Hz}, 1 \mathrm{H}), 6.47(\mathrm{~d}, J=8.5 \mathrm{~Hz}, 1 \mathrm{H}), 6.03$ $(\mathrm{d}, J=7.9 \mathrm{~Hz}, 1 \mathrm{H}), 5.37-5.33(\mathrm{~m}, J=4.0 \mathrm{~Hz}, 2 \mathrm{H}), 5.29(\mathrm{~d}, J$ $=4.4 \mathrm{~Hz}, 2 \mathrm{H}), 5.25(\mathrm{dd}, J=6.9,3.5 \mathrm{~Hz}, 2 \mathrm{H}), 5.02-4.95(\mathrm{~m}, J$ $=9.0,4.4 \mathrm{~Hz}, 2 \mathrm{H}), 4.80(\mathrm{dd}, J=10.5,3.9 \mathrm{~Hz}, 1 \mathrm{H}), 4.69(\mathrm{dd}, J$ $=10.3,4.0 \mathrm{~Hz}, 1 \mathrm{H}), 4.43-4.38(\mathrm{~m}, 2 \mathrm{H}), 4.32(\mathrm{dd}, J=12.1$, $4.0 \mathrm{~Hz}, 1 \mathrm{H}), 4.19$ (dd, $J=12.5,3.4 \mathrm{~Hz}, 1 \mathrm{H}), 4.08(\mathrm{~d}, J=7.1$ $\mathrm{Hz}, 1 \mathrm{H}), 4.03-3.99(\mathrm{~m}, J=7.4 \mathrm{~Hz}, 1 \mathrm{H}), 3.92-3.85(\mathrm{~m}, J=$ $8.5 \mathrm{~Hz}, 4 \mathrm{H}), 3.73(\mathrm{~s}, 6 \mathrm{H}), 2.09(\mathrm{~d}, J=3.9 \mathrm{~Hz}, 6 \mathrm{H}), 2.04(\mathrm{~s}$, 3H), $2.01(\mathrm{~s}, 3 \mathrm{H}), 1.97(\mathrm{~s}, 9 \mathrm{H}), 1.96-1.93(\mathrm{~m}, 9 \mathrm{H}) ;{ }^{13} \mathbf{C}\{\mathbf{1 H}\}$ NMR (75 MHz, $\left.\mathbf{C D C l}_{3}\right) \boldsymbol{\delta}$ (ppm) $170.79\left(2 \times \mathrm{C}_{\mathrm{q}}\right), 170.64(2 \mathrm{x}$ $\left.\mathrm{C}_{\mathrm{q}}\right), \quad 170.50\left(\mathrm{C}_{\mathrm{q}}\right), \quad 170.19\left(\mathrm{C}_{\mathrm{q}}\right), \quad 169.93\left(\mathrm{C}_{\mathrm{q}}\right), \quad 169.78\left(2 \times \mathrm{C}_{\mathrm{q}}\right.$, $169.60\left(2 \times \mathrm{C}_{\mathrm{q}}\right), 164.14\left(\mathrm{C}_{\mathrm{q}}\right), 157.91\left(\mathrm{C}_{\mathrm{q}}\right), 132.00(\mathrm{CH}), 104.12(2$ $x \quad \mathrm{CH}), \quad 96.00(\mathrm{CH}), \quad 95.84(\mathrm{CH}), \quad 91.84(\mathrm{CH}), \quad 75.60(\mathrm{CH})$, 73.63 $(\mathrm{CH}), 73.30(\mathrm{CH}), 72.70(\mathrm{CH}), 71.89(\mathrm{CH}), 71.58(\mathrm{CH})$, 70.59(CH), 70.22(CH), 69.54(CH), 69.14(CH), 68.67(CH), 68.09(CH), 63.00 $\left(\mathrm{CH}_{2}\right), 62.47\left(\mathrm{CH}_{2}\right), 61.57\left(\mathrm{CH}_{2}\right), 56.15(2 \mathrm{x}$ $\left.\mathrm{CH}_{3}\right), 29,85\left(\mathrm{C}_{\mathrm{q}}\right), 21.00\left(\mathrm{CH}_{3}\right), 20.81\left(\mathrm{CH}_{3}\right), 20.73\left(8 \times \mathrm{CH}_{3}\right)$; HRMS (ESI) $(\mathbf{M}+\mathbf{N a})^{+} \mathrm{m} / z$ calculated for $\mathrm{C}_{47} \mathrm{H}_{60} \mathrm{O}_{29} \mathrm{Na}$ : 1111.3118, found 1111.3125 .

$\beta$-D-Glucopyranose, $O$-2,3,4,6-tetra-O-acetyl- $\alpha-D$ glucopyranosyl-( $1 \rightarrow 4)-O-2,3,6$-tri-O-acetyl- $\alpha-D$ - glucopyranosyl- $(1 \rightarrow 4)$-, dichlorobenzoate) $[\mathbf{8 k}]$

Compound $\mathbf{8 k}$ was prepared by using the general procedure $\mathrm{B}$ (reaction time: 1h), with commercial 3,5-dichlorobenzoic acid 5m (36.7 mg, 0.19 mmol, 1.0 equiv.) and $\mathbf{1 i}$ (270.8 mg, 0.29 mmol, 1.5 equiv.), which afforded the product as a white solid $(80.6 \mathrm{mg}, \quad 38 \%$ yield after column chromatography EtOAc/Cyclohexane 1:1); TLC $\quad \mathbf{R}_{\mathbf{f}}=0.25$ (EtOAc/Cyclohexane, 1:1, $\mathrm{SiO}_{2}$ ); mp: $125.4-126.7{ }^{\circ} \mathrm{C}$; $[\boldsymbol{\alpha}]_{\mathbf{D}}{ }^{\mathbf{2 0}}=+64.0\left(\mathrm{c}, 1.00\right.$ in $\left.\mathrm{CHCl}_{3}\right)$; IR (neat, $\mathbf{~ c m}^{-\mathbf{1}}$ ) 2964, 2934, 1756, 1572, 1433, 1367, 1205, 1024; ${ }^{\mathbf{1}} \mathbf{H}$ NMR (300 MHz, $\left.\mathbf{C D C l}_{3}\right) \boldsymbol{\delta}$ (ppm) $7.88(\mathrm{~s}, 2 \mathrm{H}), 7.58(\mathrm{~s}, 1 \mathrm{H}), 5.97(\mathrm{~d}, J=7.7$ $\mathrm{Hz}, 1 \mathrm{H}), 5.44-5.35(\mathrm{~m}, 4 \mathrm{H}), 5.33$ (d, $J=4.4 \mathrm{~Hz}, 1 \mathrm{H}), 5.29$ $(\mathrm{d}, J=4.6 \mathrm{~Hz}, 1 \mathrm{H}), 5.17-5.09(\mathrm{~m}, 1 \mathrm{H}), 5.05(\mathrm{~d}, J=9.9 \mathrm{~Hz}$, $1 \mathrm{H}), 4.86(\mathrm{dd}, J=10.3,4.1 \mathrm{~Hz}, 1 \mathrm{H}), 4.75(\mathrm{dd}, J=10.3,3.9$ $\mathrm{Hz}, 1 \mathrm{H}), 4.47$ (d, $J=11.6 \mathrm{~Hz}, 2 \mathrm{H}), 4.28$ (ddd, $J=20.8,12.4$, $3.6 \mathrm{~Hz}, 2 \mathrm{H}), 4.21-4.12(\mathrm{~m}, 1 \mathrm{H}), 4.10-4.00(\mathrm{~m}, 2 \mathrm{H}), 3.93$ $(\mathrm{dd}, J=11.1,6.0 \mathrm{~Hz}, 3 \mathrm{H}), 2.15(\mathrm{~s}, 6 \mathrm{H}), 2.10(\mathrm{~s}, 3 \mathrm{H}), 2.06(\mathrm{~s}$, $3 \mathrm{H}), 2.04(\mathrm{~s}, 6 \mathrm{H}), 2.03(\mathrm{~s}, 3 \mathrm{H}), 2.01(\mathrm{~s}, 3 \mathrm{H}), 1.99(\mathrm{~s}, 6 \mathrm{H})$; ${ }^{13} \mathbf{C}\{1 \mathrm{H}\}$ NMR (75 MHz, $\left.\mathbf{C D C l}_{3}\right) \boldsymbol{\delta}(\mathbf{p p m}) 170.8\left(2 \times \mathrm{C}_{\mathrm{q}}\right)$, $170.7\left(\mathrm{C}_{\mathrm{q}}\right), 170.6\left(\mathrm{C}_{\mathrm{q}}\right), 170.5\left(\mathrm{C}_{\mathrm{q}}\right), 170.0\left(2 \times \mathrm{C}_{\mathrm{q}}\right), 169.8\left(\mathrm{C}_{\mathrm{q}}\right)$, $169.7\left(\mathrm{C}_{\mathrm{q}}\right), 169.6\left(\mathrm{C}_{\mathrm{q}}\right), 162.4\left(\mathrm{C}_{\mathrm{q}}\right), 135.7\left(2 \times \mathrm{C}_{\mathrm{q}}\right), 133.9(\mathrm{CH})$, $131.5\left(\mathrm{C}_{\mathrm{q}}\right), 128.6(2 \times \mathrm{CH}), 96.2(\mathrm{CH}), 95.8(\mathrm{CH}), 92.4(\mathrm{CH})$, $74.9(\mathrm{CH}), 73.6(\mathrm{CH}), 73.2(\mathrm{CH}), 72.6(\mathrm{CH}), 71.8(\mathrm{CH}), 70.9$ $(\mathrm{CH}), 70.6(\mathrm{CH}), 70.2(\mathrm{CH}), 69.5(\mathrm{CH}), 69.3(\mathrm{CH}), 68.7(\mathrm{CH})$, $68.0(\mathrm{CH}), 62.7\left(\mathrm{CH}_{2}\right), 62.4\left(\mathrm{CH}_{2}\right), 61.5\left(\mathrm{CH}_{2}\right), 21.1(3 \mathrm{x}$ $\left.\mathrm{CH}_{3}\right), 20.8\left(7 \times \mathrm{CH}_{3}\right) ;$ HRMS (ESI) $(\mathbf{M}+\mathbf{N a})^{+} \mathrm{m} / z$ calculated for $\mathrm{C}_{45} \mathrm{H}_{54} \mathrm{Cl}_{2} \mathrm{O}_{27} \mathrm{Na}$ 1119.2127, found 1119.2125.

2. Application of the methodology for molecular complexity

$\beta$-D-Glucopyranose, 2,3,4,6-tetraacetate $\quad 1$-[4-[[3,4,6-tri-Oacetyl-2-(acetylamino)-2-deoxy- $\beta$-D-glucopyranosyl]thio] benzoate] [9a]

According to A. Bruneau et al. procedure $^{[31]}$, a sealed tube is charged with Xantphos Pd-G3 precatalyst (2 mol\%), $N$-acetyl1-thio- $\beta$-D-aminoglucopyranose $\mathbf{1 j}(27.2 \mathrm{mg}, 0.075 \mathrm{mmol}, 1.0$ equiv.), $\quad(2 R, 3 R, 4 S, 5 R, 6 S)$-2-(acetoxymethyl)-6-((4iodobenzoyl)oxy)tetrahydro- $2 H$-pyran-3,4,5-triyl triacetate $7 \mathbf{l}$ (43.4 mg, $0.075 \mathrm{mmol}, 1$ equiv.). After Argon flushing, dioxane $(0.3 \mathrm{~mL}, 0.25 \mathrm{M})$ is added. Upon stirring the reaction mixture, $\mathrm{NEt}_{3}(0.01 \mathrm{~mL}, 0.075 \mathrm{mmol}, 1$ equiv.) is added to the medium. The reaction mixture was stirred at room temperature under Argon during 2h, which afforded the product 9a as a white solid (54.9 mg, 90\% yield after column chromatography EtOAc/Cyclohexane 5:5 $\quad \rightarrow \quad 8: 2) ; \quad$ TLC $\quad \mathbf{R}_{\mathbf{f}}=0.07$ (EtOAc/Cyclohexane, $\left.1: 1, \mathrm{SiO}_{2}\right)$; $\mathbf{m p}: 248.9-249.4{ }^{\circ} \mathrm{C}$; $[\boldsymbol{\alpha}]_{\mathbf{D}}{ }^{19}=-8.5\left(\mathrm{c}, 1.00\right.$ in $\left.\mathrm{CHCl}_{3}\right)$; IR (neat, $\mathbf{~ c m}^{-1}$ ) 2939, 1740, 1595, 1434, 1367, 1213, 1084, 1043; ${ }^{\mathbf{1}} \mathbf{H}$ NMR (300 MHz, $\left.\mathbf{C D C l}_{3}\right) \boldsymbol{\delta}(\mathbf{p p m}) 7.92(\mathrm{~d}, J=8.5 \mathrm{~Hz}, 2 \mathrm{H}), 7.48(\mathrm{~d}, J=8.5 \mathrm{~Hz}$, $2 \mathrm{H}), 5.89$ (d, $J=7.9 \mathrm{~Hz}, 1 \mathrm{H}), 5.73(\mathrm{~d}, J=9.2 \mathrm{~Hz}, 1 \mathrm{H}), 5.36-$ $5.30(\mathrm{~m}, 2 \mathrm{H}), 5.30-5.13(\mathrm{~m}, 2 \mathrm{H}), 5.13-4.99(\mathrm{~m}, 2 \mathrm{H}), 4.32$ $(\mathrm{dd}, J=12.6,4.4 \mathrm{~Hz}, 1 \mathrm{H}), 4.23-4.09$ (m, 3H), 3.93 (ddd, $J=$ 9.7, 4.5, 2.2 Hz, 1H), 3.81 (ddd, $J=10.0,5.6,2.6 \mathrm{~Hz}, 1 \mathrm{H})$, $2.10(\mathrm{~s}, 3 \mathrm{H}), 2.07(\mathrm{~s}, 3 \mathrm{H}), 2.04(\mathrm{~s}, 6 \mathrm{H}), 2.03(\mathrm{~s}, 6 \mathrm{H}), 1.98(\mathrm{~s}$, $3 \mathrm{H}), 1.95$ (s, 3H), no NH proton observed, ${ }^{13} \mathbf{C}\{\mathbf{1 H}\} \mathbf{N M R}(\mathbf{7 5}$ MHz, $\left.\mathbf{C D C l}_{3}\right) \boldsymbol{\delta}(\mathbf{p p m}) 171.2\left(\mathrm{C}_{\mathrm{q}}\right), 170.7\left(2 \times \mathrm{C}_{\mathrm{q}}\right), 170.3\left(\mathrm{C}_{\mathrm{q}}\right)$, $170.2\left(\mathrm{C}_{\mathrm{q}}\right), 169.6\left(\mathrm{C}_{\mathrm{q}}\right), 169.5\left(\mathrm{C}_{\mathrm{q}}\right), 169.4\left(\mathrm{C}_{\mathrm{q}}\right), 164.1\left(\mathrm{C}_{\mathrm{q}}\right)$, $141.9\left(\mathrm{C}_{\mathrm{q}}\right), 130.6(2 \times \mathrm{CH}), 129.6(2 \times \mathrm{CH}), 126.9\left(\mathrm{C}_{\mathrm{q}}\right), 92.5$ $(\mathrm{CH}), 85.6(\mathrm{CH}), 76.1(\mathrm{CH}), 73.5(\mathrm{CH}), 72.9(\mathrm{CH}), 72.7(\mathrm{CH})$, $70.2(\mathrm{CH}), 68.4(\mathrm{CH}), 68.0(\mathrm{CH}), 62.5\left(\mathrm{CH}_{2}\right), 61.6\left(\mathrm{CH}_{2}\right), 53.4$ $(\mathrm{CH}), 23.4\left(\mathrm{CH}_{3}\right), 20.9\left(\mathrm{CH}_{3}\right), 20.8\left(2 \times \mathrm{CH}_{3}\right), 20.7\left(4 \times \mathrm{CH}_{3}\right)$; 
HRMS (ESI) $(\mathbf{M}+\mathbf{N a})^{+} \mathrm{m} / z$ calculated for $\mathrm{C}_{35} \mathrm{H}_{43} \mathrm{NO}_{19} \mathrm{SNa}$ 836.2048, found 836.2033.

L-Cysteine, $N$-acetyl-S-[4-[[(2,3,4,6-tetra-O-acetyl- $\beta$ - $D$ -

glucopyranosyl)oxy]carbonyl]phenyl]- [9b]

According to A. Bruneau et al. procedure $^{[31]}$, a sealed tube is charged with Xantphos Pd-G3 precatalyst (4 mol\%), N-AcetylL-Cysteine (17 mg, $0.10 \mathrm{mmol}, 1.0$ equiv.), $(2 R, 3 R, 4 S, 5 R, 6 S)$ 2-(acetoxymethyl)-6-((4-iodobenzoyl)oxy)tetrahydro- $2 H$ pyran-3,4,5-triyl triacetate 7l (50 mg, $0.086 \mathrm{mmol}, 1$ equiv.). After Argon flushing, dioxane $(1 \mathrm{~mL})$ is added. Upon stirring the reaction mixture, $\mathrm{NEt}_{3}(0.129 \mathrm{mmol})$ is added to the medium. The reaction mixture was stirred at $60{ }^{\circ} \mathrm{C}$ during $20 \mathrm{~min}$, which afforded the product $\mathbf{9 b}$ as a beige solid $(35.5 \mathrm{mg}, 67 \%$ yield after column chromatography $\mathrm{MeOH} / \mathrm{DCM} 1: 9) ; \mathbf{R}_{\mathbf{f}}(5 \%$ $\mathrm{MeOH} / \mathrm{DCM})=0.29 ; \mathbf{m p :} 152-160{ }^{\circ} \mathrm{C} ;[\boldsymbol{\alpha}]_{\mathbf{D}}{ }^{25}=-50.00(\mathrm{c}$ $0,002 \mathrm{CH}_{2} \mathrm{Cl}_{2}$ ); IR (neat, $\mathbf{c m}^{-1}$ ) 1752, 1738, 1595, 1235, 1214, 1087, 1054, 1033 ; ${ }^{1}$ H NMR (300 MHz, DMSO) $\delta$ (ppm) $7.77(\mathrm{~d}, J=8.3 \mathrm{~Hz}, 2 \mathrm{H}), 7.66-7.61(\mathrm{~m}, 1 \mathrm{H}), 7.42(\mathrm{~d}, J$ $=8.4 \mathrm{~Hz}, 2 \mathrm{H}), 6.16(\mathrm{~d}, J=8.2 \mathrm{~Hz}, 1 \mathrm{H}), 5.53(\mathrm{t}, J=9.6 \mathrm{~Hz}$, $1 \mathrm{H}), 5.17-5.09(\mathrm{~m}, 1 \mathrm{H}), 5.03(\mathrm{t}, J=9.7 \mathrm{~Hz}, 1 \mathrm{H}), 4.33-4.25$ $(\mathrm{m}, J=9.2 \mathrm{~Hz}, 1 \mathrm{H}), 4.20(\mathrm{dd}, J=12.2,4.8 \mathrm{~Hz}, 1 \mathrm{H}), 4.02(\mathrm{~d}, J$ $=12.4 \mathrm{~Hz}, 1 \mathrm{H}), 3.59-3.50(\mathrm{~m}, 1 \mathrm{H}), 3.36-3.21(\mathrm{~m}, 2 \mathrm{H}), 2.08$ (s, 3H), 2.00 (d, $J=1.2 \mathrm{~Hz}, 6 \mathrm{H}), 1.98(\mathrm{~s}, 3 \mathrm{H}), 1.95(\mathrm{~s}, 3 \mathrm{H})$; ${ }^{13} \mathbf{C}\{1 \mathrm{H}\}$ NMR (75 MHz, DMSO) $\delta(\mathbf{p p m}) \quad 172.88\left(\mathrm{C}_{\mathrm{q}}\right)$, $170.87\left(\mathrm{C}_{\mathrm{q}}\right), \quad 170.31\left(2 \times \mathrm{C}_{\mathrm{q}}\right), \quad 170.09\left(2 \times \mathrm{C}_{\mathrm{q}}\right), 164.14\left(\mathrm{C}_{\mathrm{q}}\right)$, $147.14\left(\mathrm{C}_{\mathrm{q}}\right), 130.25(2 \times \mathrm{CH}), 126.48(2 \times \mathrm{CH}), 124.07\left(\mathrm{C}_{\mathrm{q}}\right)$, 92.06(CH), $72.02(2 \mathrm{x} \quad \mathrm{CH}), \quad 70.48(\mathrm{CH}), \quad 68.06(\mathrm{CH})$, 61.71 $\left(\mathrm{CH}_{2}\right)$, 53.90(CH), 34.92( $\left.\mathrm{CH}_{2}\right), 22.97\left(\mathrm{CH}_{3}\right), 20.81\left(\mathrm{CH}_{3}\right)$, 20.69 $\left(\mathrm{CH}_{3}\right), 20.62\left(\mathrm{CH}_{3}\right), 20.54\left(\mathrm{CH}_{3}\right)$; HRMS (ESI) $(\mathbf{M}+$ $\mathrm{Na})^{+} m / z$ calculated for $\mathrm{C}_{26} \mathrm{H}_{31} \mathrm{NO}_{14} \mathrm{NaS}$ : 636.1363, found 636.1370 .

\section{Extension of the methodology for molecular diversity}

3.1 Procedure for the synthesis of 2-acetamidoethyl 3,5dichlorobenzoate [10a] and its characterization data Compound 10a was prepared by using the general procedure (reaction time: 1h), with commercial 3,5-dichlorobenzoic acid $5 \mathrm{~m}(36.7 \mathrm{mg}, 0.19 \mathrm{mmol}, 1.0$ equiv.) and commercial $\mathrm{N}$-acetylcysteamine (34.3 mg, $0.29 \mathrm{mmol}, 1.5$ equiv.), which afforded the product as a white solid $(35.8 \mathrm{mg}, 68 \%$ yield after column chromatography EtOAc/Cyclohexane 1:1 -> 7:3); TLC $\mathbf{R}_{\mathbf{f}}=0.13\left(\right.$ EtOAc/Cyclohexane, $\left.1: 1, \mathrm{SiO}_{2}\right)$; mp: $139.0-$ $139.5^{\circ} \mathrm{C} ;[\boldsymbol{\alpha}]_{\mathbf{D}}{ }^{20}=-5.1$ (c, 1.00 in $\mathrm{CHCl}_{3}$ ); IR (neat, $\mathbf{~ c m}^{-\mathbf{1}}$ ) 3293, 3078, 1728, 1642, 1570, 1432, 1385, 1362, 1274; ${ }^{\mathbf{1}} \mathbf{H}$ NMR (300 MHz, CDCl $) \boldsymbol{\delta}$ (ppm) $7.90(\mathrm{~d}, J=1.9 \mathrm{~Hz}, 2 \mathrm{H})$, $7.56(\mathrm{t}, J=2.0 \mathrm{~Hz}, 1 \mathrm{H}), 5.99-5.77(\mathrm{~m}, 1 \mathrm{H}, \mathrm{NH}$ proton $), 4.43$ (t, $J=5.3 \mathrm{~Hz}, 2 \mathrm{H}), 3.66(\mathrm{dd}, J=10.9,5.5 \mathrm{~Hz}, 2 \mathrm{H}), 2.03(\mathrm{~s}$, $3 \mathrm{H}) ;{ }^{13} \mathbf{C}\{1 \mathrm{H}\}$ NMR (75 $\left.\mathbf{M H z}, \mathbf{C D C l}_{3}\right) \delta(\mathbf{p p m}) 170.7\left(\mathrm{C}_{\mathrm{q}}\right)$, $164.5\left(\mathrm{C}_{\mathrm{q}}\right), 135.6\left(2 \times \mathrm{C}_{\mathrm{q}}\right), 133.2(\mathrm{CH}), 132.7\left(\mathrm{C}_{\mathrm{q}}\right), 128.2(2 \mathrm{x}$ $\mathrm{CH}), 64.8\left(\mathrm{CH}_{2}\right), 39.0\left(\mathrm{CH}_{2}\right), 23.4\left(\mathrm{CH}_{3}\right)$; HRMS (ESI) $(\mathbf{M}+$ $\mathrm{Na})^{+} \mathrm{m} / \mathrm{z}$ calculated for $\mathrm{C}_{11} \mathrm{H}_{11} \mathrm{NO}_{3} \mathrm{Cl}_{2} \mathrm{Na} 298.0014$, found 298.0025 .

\section{Synthesis of the starting thiosugars $1 \mathrm{~g}$}

$\beta$-D-Glucopyranose, 2,4,6-tris-O-[(1,1-dimethylethyl) dimethylsilyl]-1-thio-1g

$\mathrm{NaOMe}(0.5 \mathrm{M}$ sol. in $\mathrm{MeOH}, 6 \mathrm{ml}, 1.5 \mathrm{eq})$ was added to a solution thiosugar 1a $(750 \mathrm{mg}, 2 \mathrm{mmol})$ in $\mathrm{MeOH}(28 \mathrm{ml})$ and stirred at RT for 30 min under Ar atmosphere. The solution was neutralized using Dowex® 50WX8 100-200 (H), filtered, concentrated and used without further purification.
To the polyhydroxy product in anhydrous DMF (14 ml), TBDMSCl ( $3 \mathrm{~g}, 20 \mathrm{mmmol}, 10$ equiv.) and imidazole ( $3.3 \mathrm{~g}$, $48 \mathrm{mmmol}, 24$ equiv.) were added and stirred at RT for overnight under Ar atmosphere. TLC showed no more starting material and also showed products with different number of TBS protecting groups (three and four according to HRMS $(\mathrm{ESI}+))$. The reaction was quenched with $\mathrm{MeOH}(10 \mathrm{ml})$ then extracted with pentane $(3 \times 15 \mathrm{ml})$. The pentane layers were collected and dried over $\mathrm{MgSO}_{4}$, filtered, concentrated under reduced pressure then purified by column chromatography using (1-2\% EtOAc in pentane). Out of all fractions, only one pure fraction was obtained as colorless oil $(72 \mathrm{mg})$ in $7 \%$ yield.

$\mathbf{R}_{\mathbf{f}}=0.67$ (using $10 \%$ EtOAc in cyclohexane); $[\boldsymbol{\alpha}]_{\mathbf{D}}{ }^{\mathbf{1 5}}=-10(\mathrm{c}$, 0.5 in $\mathrm{CHCl}_{3}$ ); IR (neat, $\mathbf{c m}^{-1}$ ) 2956.90, 2926.87, 2855.56, 1258.66, 1151.15, 835.71, 777.26, 670.96; ${ }^{1} \mathbf{H}$ NMR (400 MHz, $\left.\mathbf{C D C l}_{3}\right) \delta 4.71(\mathrm{dd}, \mathrm{J}=8.6,5.3 \mathrm{~Hz}, 1 \mathrm{H}, \mathrm{H}-1), 3.94(\mathrm{dd}$, $\mathrm{J}=10.3,4.9 \mathrm{~Hz}, 1 \mathrm{H}, \mathrm{H}-6), 3.86(\mathrm{dd}, \mathrm{J}=10.3,6.6 \mathrm{~Hz}, 1 \mathrm{H}, \mathrm{H}-$ $\left.6^{\prime}\right), 3.72(\mathrm{t}, \mathrm{J}=5.6 \mathrm{~Hz}, 1 \mathrm{H}, \mathrm{H}-4), 3.68(\mathrm{~d}, \mathrm{~J}=5.4 \mathrm{~Hz}, 1 \mathrm{H}, \mathrm{H}-2)$, $3.67-3.62(\mathrm{~m}, 1 \mathrm{H}, \mathrm{H}-3), 3.60(\mathrm{dd}, \mathrm{J}=6.8,5.0 \mathrm{~Hz}, 1 \mathrm{H}, \mathrm{H}-5)$, $3.11(\mathrm{~d}, \mathrm{~J}=4.7 \mathrm{~Hz}, 1 \mathrm{H}, \mathrm{OH}), 2.42(\mathrm{~d}, \mathrm{~J}=8.5 \mathrm{~Hz}, 1 \mathrm{H}, \mathrm{SH})$, $0.94\left(\mathrm{~s}, 6 \mathrm{H}, 2 \mathrm{XCH}_{3}\right), 0.92\left(\mathrm{~s}, 9 \mathrm{H}, 3 \mathrm{XCH}_{3}\right), 0.90(\mathrm{~s}, 12 \mathrm{H}$, $\left.4 \mathrm{XCH}_{3}\right), 0.18\left(\mathrm{~s}, 3 \mathrm{H}, \mathrm{CH}_{3}\right), 0.16\left(\mathrm{~s}, 3 \mathrm{H}, \mathrm{CH}_{3}\right), 0.14(\mathrm{~d}, \mathrm{~J}=2.8$ $\left.\mathrm{Hz}, 6 \mathrm{H}, 2 \mathrm{XCH}_{3}\right), 0.09\left(\mathrm{~s}, 6 \mathrm{H}, 2 \mathrm{XCH}_{3}\right) ;{ }^{13} \mathbf{C}\{\mathbf{1 H}\}$ NMR (101 MHz, $\mathbf{C D C l}_{3}$ ): $\delta 79.96(\mathrm{C} 1), 78.32$ (C3), 77.55 (C2), 77.22 (C4), 72.78 (C5), 65.21 (C6), $26.44\left(\mathrm{SiC}\left(\mathrm{CH}_{3}\right)_{3}\right), 26.23$ $\left(\mathrm{SiC}\left(\mathrm{CH}_{3}\right)_{3}\right), 26.01\left(\mathrm{SiC}\left(\mathrm{CH}_{3}\right)_{3}\right), 18.64\left(\mathrm{SiC}\left(\mathrm{CH}_{3}\right)_{3}\right), 18.41$ $\left(\mathrm{SiC}\left(\mathrm{CH}_{3}\right)_{3}\right), 18.27\left(\mathrm{SiC}\left(\mathrm{CH}_{3}\right)_{3}\right),-3.18\left(\mathrm{SiCH}_{3}\right),-3.27\left(\mathrm{SiCH}_{3}\right)$, -3.47 $\left(\mathrm{SiCH}_{3}\right),-3.57\left(\mathrm{SiCH}_{3}\right),-5.20\left(\mathrm{SiCH}_{3}\right),-5.23\left(\mathrm{SiCH}_{3}\right)$. HRMS (ESI) $\left(\mathbf{M}+\mathbf{N H}_{4}\right)^{+} \mathrm{m} / z$ calculated for $\mathrm{C}_{24} \mathrm{H}_{58} \mathrm{NO}_{5} \mathrm{SSi}_{3}{ }^{+}$ 556.3338 , found 556.3353 .

\section{ASSOCIATED CONTENT}

\section{Supporting Information}

The Supporting Information is available free of charge on the ACS Publications website.

Full optimization table, NMR spectra of all synthesized compounds and HPLC chromatograms.

\section{AUTHOR INFORMATION}

\section{Corresponding Author}

*samir.messaoudi@u-psud.fr

\section{Author Contributions}

The manuscript was written through contributions of all authors. / All authors have given approval to the final version of the manuscript.

\section{Funding Sources}

Any funds used to support the research of the manuscript should be placed here (per journal style).

Notes

Any additional relevant notes should be placed here.

\section{ABBREVIATIONS}

\section{ACKNOWLEDGMENT}

Authors acknowledge support of this project by CNRS, University Paris-Sud, ANR (ANR-15-CE29- 0002), and by la Ligue Contre le Cancer through an Equipe Labellisée 2014 grant. We also thank the Algerian Ministry of Education and Research for a fellowship (Profas B+) to Nedjwa Bennai. Our laboratory is a member of the 
Laboratory of Excellence LERMIT supported by a grant (ANR10-LABX-33)

\section{REFERENCES}

(1) (a) Varki, A. Biological roles of glycans, Glycobiology, 2017, 27, 3-49 (b) Gabius, H.-J. (2009) The Sugar Code: Fundamentals of Glycosciences, Wiley-Blackwell, Weinheim, Germany. (c) Varki, A.; Cummings, R.; Esko, J.; Freeze, H.; Hart, G.; Marth, J. (1999) Essentials of Glycobiology, Cold Spring Harbor Laboratory Press, Cold Spring Harbor, NY.

(2) (a) Smith, A. B. III; Rivero, R. A. Total synthesis of (+)phyllanthoside, J. Am. Chem. Soc., 1987, 109, 1272-1274. (b) Pettit, G.; Cragg, G. M.; Gust, D.; Brown, P. The isolation and structure of phyllanthostatins 2 and 3, Canadian Journal of Chemistry, 1982, 60, 544-546.

(3) Marwan, A. G.; Nagel, C.W. Identification of the Hydroxycinnamtic Acid Derivatives in Cranberries J. Food Sci., 1986, 51, 1069-1070.

(4) Zhao, C.-X.; Li, B. Q.; Shao, Z. X.; Li, D-H.; Jing, Y. Q.; Li, Z. L. $\mathrm{Hu}$, H. M. Cephasinenoside A, a new cephalotane diterpenoid glucoside from Cephalotaxus sinensis. Tetrahedron Lett. 2019, 60, $151-154$.

(5) (a) Xu, M.; Zhang, M.; Zhang, Y.-J.; Yang, C.-R. New Acylated Secoiridoid Glucosides from Gentiana straminea (Gentianaceae) Helvetica Chimica Acta 2009, 92, 321-327 (b) Rho, T.; Jung, M.; Lee, M.-W.; Chin, Y.-W.; Yoon, K.-D. Efficient methods for isolating five phytochemicals from Gentiana macrophylla using highperformance countercurrent chromatography. J Sep Sci. 2016, 39, $4723-4731$

(6) Ma, Y.; Gao, S.; Hu, M. Quantitation of celecoxib and four of its metabolites in rat blood by UPLC-MS/MS clarifies their blood distribution patterns and provides more accurate pharmacokinetics profiles J Chromatogr B, 2015, 1001, 202-211.

(7) Kim, Y.-J.; Wang, P. ; Navarro-Villalobos, M. ; Rohde, B. D. ; Mark, J.; Gin, D. Y. Synthetic Studies of Complex Immunostimulants from Quillaja saponaria: Synthesis of the Potent Clinical Immunoadju-vant QS-21Aapi, J. Am. Chem. Soc. 2006, 11906 11915.

(8) Su, X.; Surry, D. S.; Spandl, R. J.; Spring, D. R. Total Synthesis of Sanguiin H-5, Org. Lett. 2008, 10, 2593-2596

(9) Smith III, A. B. ; Rivero, R. A. ; Hale, K. J.; Vaccaro, H. A. Phyllanthoside-phyllanthostatin synthetic studies. 8. Total synthesis of (+)-phyllanthoside. Development of the Mitsunobu glycosyl ester protocol J. Am. Chem. Soc. 1991, 113, 2092-2112

(10) Shen, L.; Cai, K.; Yu, J.; Cheng, J. Novel Liposomal Azido Mannosamine Lipids on Metabolic Cell Labeling and Imaging via Cu-Free Click Chemistry, Bioconjugate Chem. 2019, 30, 2317-2322

(11) Stachulski, A. V.; Harding, J. R.; Lindon, J. C.; Maggs, J. L.; Park, B. K.; Wilson, I. D. Acyl Glucuronides: Biological Activity, Chemical Reactivity, and Chemical Synthesis, J. Med. Chem. 2006, 49, 6931-6945

(12) Wietholtz, H.; Marschall, H. U.; Reuschenbach, R.; Matern, H.; Matern, S. Urinary excretion of bile acid glucosides and glucuronides in extrahepatic cholestasis Hepatology 1991, 13, 656-662.

(13) Calvaresi, E. C.; Hergenrother, P. J. Glucose conjugation for the specific targeting and treatment of cancer, Chem Sci. 2013, 4, 23192333

(14) (a) Liu, D.-Z.; Sinchaikul, S.; Reddy, P.V.G.; Chang, M.-Y.; Chen, S.-T. Synthesis of 2'-paclitaxel methyl 2-glucopyranosyl succinate for specific targeted delivery to cancer cells Bioorg Med Chem Lett, 2007, 17, 617-620 (b) Lin, Y.-S.; Tungpradit, R.; Sinchaikul, S.; An, F.-M.; Liu, D.-Z.; Phutrakul, S.; Chen, S.-T. Targeting the deliv- ery of glycan-based paclitaxel prodrugs to cancer cells via glucose transporters, J. Med. Chem., 2008, 51, 7428-7441 (c) Ma, J.; Liu, H.; Xi, Z.; Hou, J., Li, Y.; Niu, J.; Liu, T.; Bi, S.; Wang, X.; Wang, C.; Wang, J.; Xie, S.; Wang, P.G. Protected and De-protected Platinum(IV) Glycoconjugates With GLUT1 and OCT2-Mediated Selective Cancer Targeting: Demonstrated Enhanced Transporter-Mediated Cytotoxic Properties in vitro and in vivo. Front. Chem., 2018, 6, 1-15

(15) Cao, J.; Cui, S.; Li, S.; Du, C.; Tian, J.; Wan, S.; Qian, Z.; Gu, Y.; Chen, W. R.; Wang, G. Targeted cancer therapy with a 2deoxyglucose-based adriamycin complex, Cancer Res. 2013, 73, 1362-1373

(16) (a) Iglesias-Guerra, F. ; Candela, J.I. ; Bautista, J. ; Alcudia, F.; Vega-Pérez, J.-M. Alkylating agents from sugars. Alkyl hexopyranoside derivatives as carrier systems for chlorambucil Carbohydr Res, 1999, 316, 71-84 (b)Halmos, T.; Santarromana, M.; Antonakis, K.; Scherman, D. Synthesis of glucose-chlorambucil derivatives and their recognition by the human GLUT1 glucose transporter, Eur. J. Pharmacol., 1996, 318, 477-484

(17) (a) Patra, M.; Awuah, S.G.; Lippard, S.J. Chemical approach to positional isomers of glucose-platinum conjugates reveals specific cancer targeting through glucose-transporter-mediated uptake in vitro and in vivo J. Am. Chem. Soc., 2016, 138, 12541-1255; (b) Gao, X.; Liu, S.; Shi, Y.; Huang, Z.; Yi, M.; Mi, Q.; Yang, J.; Gao, Q. Mechanistic and biological characteristics of different sugar conjugated 2methyl malonatoplatinum(II) complexes as new tumor targeting agents Eur. J. Med. Chem., 2017, 125, 372-384 (c) Patra, M.; Johnstone, T.C.; Suntharalingam, K.; Lippard, S.J. A potent glucoseplatinum conjugate exploits glucose transporters and preferentially accumulates in cancer cells Angew. Chem. Int. Ed. 2016, 55, 25502554

(18) Iglesias-Guerra, F.; Romero, I.; Alcudia, F.; Vega-Pérez, J.M. Alkylating agents from sugars. Cyclophosphamides derived from 2amino-2-deoxy-D-allose Carbohydr. Res., 1998, 308, 57-62.

(19) Sakaguchi, K.; Green, M.; Stock, N.; Reger, T. S.; Zunic, J.; King, C. Glucuronidation of carboxylic acid containing com-pounds by UDP-glucuronosyltransferase isoforms. Arch. Biochem. Biophys. 2004, 424, 219-225

(20) Smith III, A. B.; Hale, K. J.; Rivero, R. A. An efficient synthesis of glycosyl esters exploiting the mitsunobu reaction Tetrahedron Letters, 1986, 27, 5813-5816

(21) Perrie, J. A.; Harding, J. R.; Holt, D. W.; Johnston, A.; Meath, P.; Stachulski, A. V. Effective Synthesis of 1- $\alpha$-AcylGlucuronides by Selective Acylation Org. Lett. 2005, 7, 2591-2594

(22) For selected examples, see: (a) Weber, J. Svatunek, D.; Krauter, S.; Tegl, G.; Hametner, C. Kosmab, P.; Mikula, H. 2-OBenzyloxycarbonyl protected glycosyl donors: a revival of carbonatemediated anchimeric assistance for diastereoselective glycosylation Chem. Commun., 2019, 55, 12543-12546 ; (b) Chen, Y.; Lu, H.; Chen, Y.; Yu,, W.; Dai, H.; Pan, X. Improved Synthesis of 1-O-Acyl- $\beta$-DGlucopyranose Tetraacetates Molecules 2017, 22, 662; (c) Yanli Cui, Y.; Xu, M.; Yao, W.; Mao, J. Room-temperature ionic liquids enhanced green synthesis of $\boldsymbol{\beta}$-glycosyl 1-ester Carbohydrate Res. 2015 407, 51e54; (d) Matsuo, K.; Nishikawa, K. Shindo, M. Stereoselective synthesis of $\beta$-glycosyl esters of cis-cinnamic acid and its derivatives using unprotected glycosyl donors, Tetrahedron Lett. 2011, 52, 56885692; (e) Crich, D.; Cai, F. Stereocontrolled Glycoside and Glycosyl Ester Synthesis. Neighboring Group Participation and Hydrogenolysis of 3-(2'-Benzyloxyphenyl)-3,3-dimethylpropanoates Org. Lett. 2007, $9,1613-1615$.

(23) (a) Wang, H.-Y.; Simmons, C. J.; Zhang, Y.; Smits, A. M.; Balzer, P. G.; Wang, S.; Tang, W. Chiral Catalyst-Directed Dynamic Kinetic Diastereoselective Acylation of Anomeric Hydroxyl Groups and a Controlled Reduction of the Glycosyl Ester Products Org. Lett. 2017, 19, 508-511; (b) Wang, H.-Y.; Yang, K.; Yin, D.; Liu, C.; Glazier, D. A.; Tang, W. "Chiral Catalyst-Directed Dynamic Kinetic 
Diastereoselective Acylation of Lactols for De Novo Synthesis of Carbohydrate." Org. Lett. 2015, 17, 5272-5275.

(24) Yang, T.; Zhu, F.; Walczak, M.A. Stereoselective oxidative glycosylation of anomeric nucleophiles with alcohols and carboxylic acids Nat. Commun., 2018, 9, 1-9

(25) (a) Floyd, N.; Vijayakrishnan, B.; Koeppe, J.; Davis, B.; Thiyl glycosylation of olefinic proteins: S-linked glycoconjugate synthesis. Angew. Chem. Int. Ed. 2009, 48, 7798-7802. (b) Goncalo, J. L. B.; David, P. G.; Benjamin, G. D. The Direct Formation of Glycosyl Thiols from Reducing Sugars Allows One- Pot Protein Glycoconjugation, Angew. Chem., Int. Ed. 2006, 45, 4007-4011

(26) Chabrier, A.; Bruneau, A.; Benmahdjoub, S.; Benmerad, B.; Belaid, S.; Brion, J.-D.; Alami, M.; Messaoudi, S. Stereoretentive copper catalyzed directed thioglycosylation of $\mathrm{C}(\mathrm{sp} 2)-\mathrm{H}$ bonds of benzamides, Chem. Eur. J, 2016, 22, 15006-15010

(27) Barsu, N.; Kumar Bolli, S.; Sundararaju, B. Cobalt catalyzed carbonylation of unactivated $\mathrm{C}\left(\mathrm{sp}^{3}\right)-\mathrm{H}$ bonds Chem. Sci. 2017, 8, 2431-2435

(28) (a) Alexander, S. A.; Kyia, C.; Schiesser, C. H. Nitroxides as anti-biofilm compounds for the treatment of Pseudomonas aeruginosa and mixed-culture biofilms Org. Biomol. Chem., 2015, 13, 4751 - 4759, (b) Zhang, H.; Zhu, P.; Liu, J.; Yang, X.; Xu, S.; Yao, H.; Jiang, J.; Ye, W.; Wu, X.; Xu, J. Synthesis and antitumor activity of novel 3-oxo-23-hydroxybetulinic acid derivatives, Eur. J. Med. Chem., 2014, 87, $159-167$.

(29) (a) Pedersen, C. M.; Nordstrøm, L. U.; Bols, M. "Super Armed" Glycosyl Donors: Conformational Arming of Thioglycosides by Silylation, J. Am. Chem. Soc. 2007, 129, 9222-9235. (b) Heuckendorff, M. Premathilake, H. D. Pornsuriyasak, P.; Madsen, A.; Pedersen, C. M.; Bols, M.; Demchenko, A. V. Superarming of Glycosyl Donors by Combined Neighboring and Conformational Effects. Org. Lett. 2013, 15, 4904-4907

(30) Axer, A.; Hermann, S.; Kehr, G.; Clases, D.; Karst, U.; FischerRiepe, L.; Roth, J.; Fobker, M.; Schafers, M.; Gilmour, R.; Faust, A." Harnessing the Maltodextrin Transport Mechanism for Targeted Bacterial Imaging: Structural Requirements for Improved in vivo Stability in Tracer Design. ChemMedChem 2018, 13, 241-250
(31) (a) Bruneau, A.; Roche, M.; Hamze, A.; Brion, J.-D.; Alami, M.; Messaoudi, S. "Stereoretentive Palladium-Catalyzed Arylation, Alkenylation and Alkynylation of 1-Thiosugars and Thiols Using Aminobiphenyl Palladacycle Precatalyst at Room Temperature", Chem. Eur. J, 2015, 21, 8375 - 8379; (b) (b) Montoir, D.; Amoura, M.; Ababsa, Z.E.-A.; Vishwanath, T. M.; Yen-Pon, E.; Robert, V.; Beltramo, M. ; Piller, V. ; Alami, M. ; Aucagne, A.; Messaoudi, S. Synthesis of Aryl-Thioglycopeptides Through Chemoselective PdMediated Conjugation, Chem.Sci. 2018, 9, 8753-8759.

(32) Nobuyoshi, K.; Yamada, S.; Kimura, T. Thermal Decomposition of Silver Carbonate: Phenomenology and Physicogeometrical Kinetics, J. Phys. Chem. C. 2013, 117, 326-336.

(33) Selected exempels (a) Robert C. Smith, Villel D. Reed \& William E. Hill, Oxidation Of Thiols By Copper(II). Phosphorus Sulfur, 1994, 90, 147-154. (b) Kreitman,G. Y.; Danilewicz, J. C.; Jeffery, D. W.; Elias, R. J. Copper(II)-Mediated Hydrogen Sulfide and Thiol Oxidation to Disulfides and Organic Polysulfanes and Their Reductive Cleavage in Wine: Mechanistic Elucidation and Potential Applications, J. Agric. Food Chem. 2017, 65, 2564-2571. (c) Ngamchuea, K.; Batchelor- McAuley, C.; Compton, R. G. The Copper(II)- Catalyzed Oxidation of Glutathione Chem. Eur. J, 2016, 24, 15937-15944

(34) Bell, R.A.; Kramer, J.R. Structural chemistry and geochemistry of silver-sulfur compounds: Critical review, Environ. Toxicol. Chem., 1999, 18, 9-22.

(35) An initial version of this manuscript was deposited in the preprint repository ChemRxiv: Messaoudi, S.; Bennai, N.; Chabrier, A.; Fatthalla, M.; Yen-Pon, E.; Tran, C.; belkadi, M.; Alami, M.; Grimaud, L. Reversing Reactivity: Stereoselective Desulfurative $\beta$-OGlycosylation of Anomeric Thiosugars with Carboxylic Acids Under Copper or Cobalt Catalysis. 2019, ChemRxiv. Preprint. https://doi.org/10.26434/chemrxiv.11421576.v1 
1,2 trans-selective 0-glycosylation of anomeric thiosugars

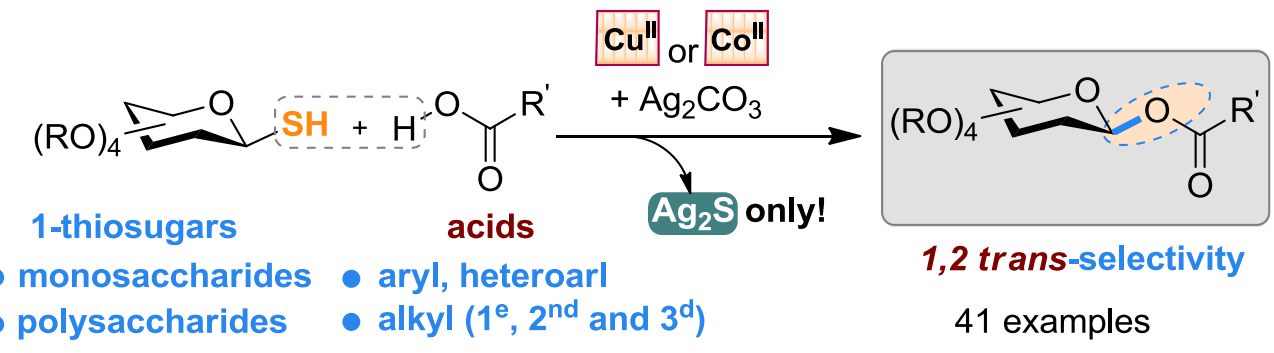

